Supporting Information

\title{
Antiviral Terpenoid Constituents of Ganoderma pfeifferi
}

\author{
Timo H. J. Niedermeyer, ${ }^{\dagger, \ddagger}$ Ulrike Lindequist, ${ }^{\dagger, \ddagger}$ Renate Mentel, ${ }^{\S}$ Dirk Gördes, ${ }^{\perp}$ \\ Enrico Schmidt, ${ }^{\perp}$ Kerstin Thurow, ${ }^{\perp}$ and Michael Lalk ${ }^{\dagger, \ddagger * *}$ \\ ${ }^{\dagger}$ Institute of Pharmacy, \\ Ernst-Moritz-Arndt-University, Friedrich-Ludwig-Jahn-Str. 17, 17487 Greifswald, Germany \\ ¥ Ganomycin Society for Biomedical Research $m b H$, \\ Walther-Rathenau-Str. 49a, 17489 Greifswald, Germany \\ $\S$ Friedrich-Löffler-Institute of Medical Microbiology, \\ Ernst-Moritz-Arndt-University, Martin-Luther-Str. 6, 17487 Greifswald, Germany \\ ${ }^{\perp}$ College of Computer Science and Electrical Engineering, Institute of Automation, \\ University of Rostock, Richard-Wagner-Str. 31, 18119 Rostock, Germany \\ * To whom correspondence should be addressed. \\ Tel: +49-3834-864867. Fax: +49-3834-864885. E-mail: lalk@uni-greifswald.de
}




\section{Contents}

1 Experimental Section $\quad 1$

2 Fractionation Sceme 2

3 Spectra of Isolated Compounds $\quad 3$

3.1 Ergosta-7,22-diene-3-one . . . . . . . . . . . . . . . 4

3.2 Ganoderal A . . . . . . . . . . . . . . . . . . . . . . 7

3.3 Ergosta-4,6,8(14),22-tetraene-3-one . . . . . . . . . . . . . . . . 10

3.4 Ergosta-7,22-diene-3 $\beta$-ol . . . . . . . . . . . . . . . . . . 14

3.5 Lucialdehyde $\mathrm{D}^{*} \ldots \ldots \ldots \ldots \ldots \ldots \ldots \ldots$

3.6 Lucialdehyde B . . . . . . . . . . . . . . . . . . . . . . . . . . . . . 29

3.7 Ganoderol A / Ganodermenonol . . . . . . . . . . . . . . . . . . 32

$3.85 \alpha, 8 \alpha$-Epidioxyergosta-6,22-diene- $3 \beta$-ol $\ldots \ldots \ldots \ldots$

3.9 Ganoderol B / Ganodermadiol . . . . . . . . . . . . . . . . . . . . . . 40

3.10 Applanoxidic acid C . . . . . . . . . . . . . . . . . . . . 43

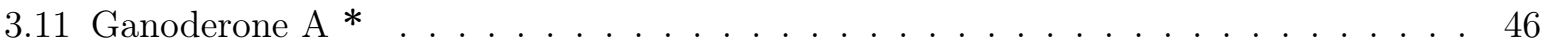

3.12 Ganoderone $\mathrm{C}^{*} \ldots \ldots \ldots \ldots \ldots \ldots$

3.13 Applanoxidic acid $G \ldots \ldots \ldots$. . . . . . . . . . . . . . . 67

3.14 Applanoxidic acid A . . . . . . . . . . . . . . . . . . 71

4 References $\quad 74$ 


\section{Experimental Section}

\section{Additional Infomation to General Experimental Procedures}

The gradients of the solvents $\mathrm{MeOH}$ (A) and water (B) used for semi-preparative HPLC are summarized in Table 1-1 (flow $15 \mathrm{~mL} / \mathrm{min}$ ).

\begin{tabular}{ll} 
& Gradient \\
\hline HPLC 1 & $0 \%$ B for 19 min \\
HPLC 2 & $2 \%$ B in 8 min to $0 \% \mathrm{~B}$, holding for $11 \mathrm{~min}$ \\
HPLC 3 & $8 \% \mathrm{~B}$ in 12 min to $0 \% \mathrm{~B}$, holding for $8 \mathrm{~min}$ \\
HPLC 4 & $10 \%$ B holding for $3 \mathrm{~min}$, in 12 min to $0 \% \mathrm{~B}$, holding for 3 min \\
HPLC 5 & $40 \%$ B in 17 min to $30 \% \mathrm{~B}$, in 0.5 min to $0 \% \mathrm{~B}$, holding for 6 min \\
HPLC 6 & $20 \%$ B in 13 min to $0 \% \mathrm{~B}$, holding for 1 min \\
HPLC 7 & $30 \%$ B in 9 min to $9 \% \mathrm{~B}$, in 0.1 min to $0 \% \mathrm{~B}$, holding for 2 min increasing \\
& flow gradually to $18 \mathrm{~mL} / \mathrm{min}$ \\
HPLC 8 & $35 \%$ B in 8.5 min to $20 \% \mathrm{~B}$, in 0.25 min to $0 \% \mathrm{~B}$, holding for 2 min increasing \\
& flow gradually to $18 \mathrm{~mL} / \mathrm{min}$
\end{tabular}

Table 1-1 Gradients used in semi-preparative HPLC 


\section{Fractionation Sceme}

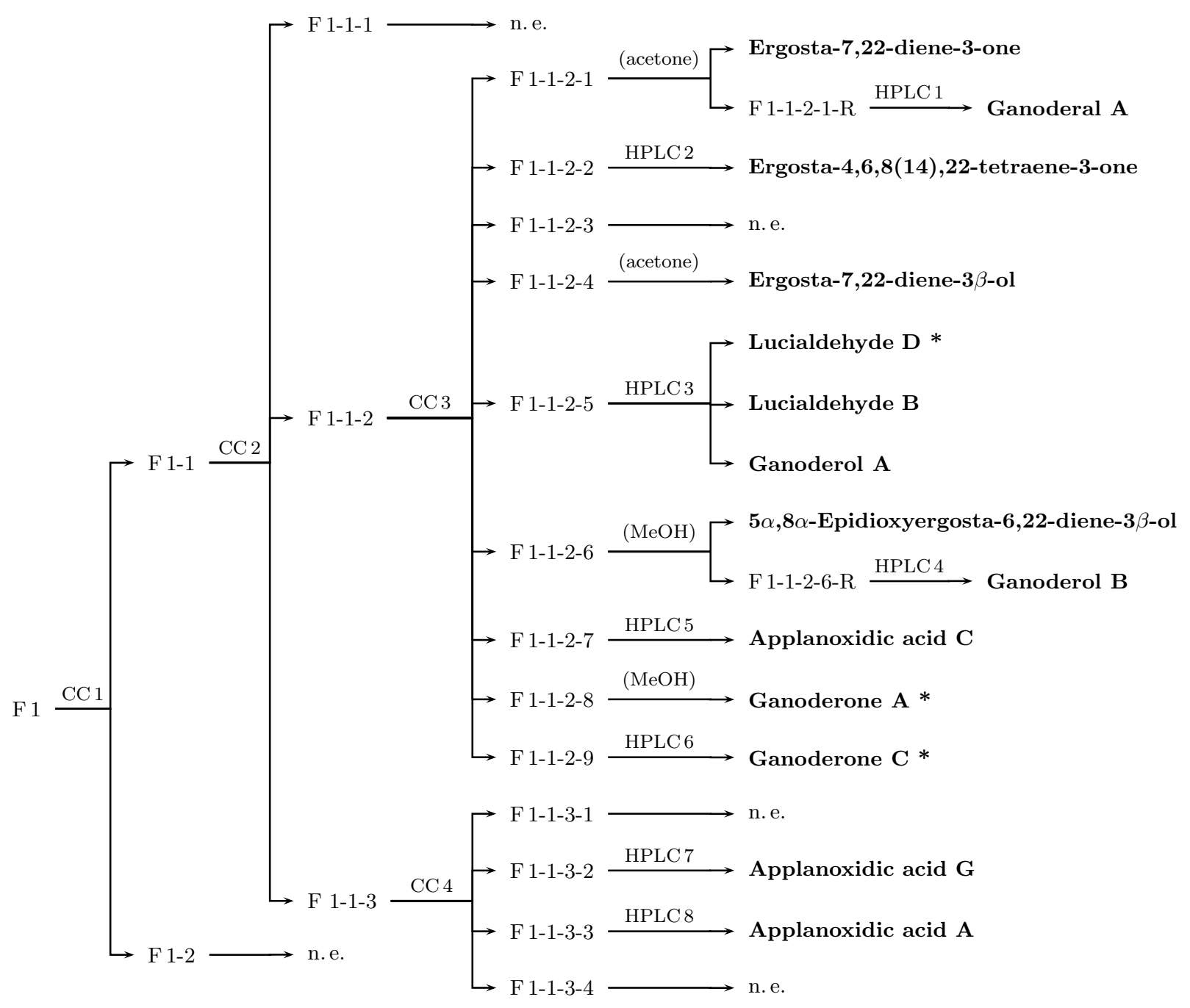

Figure 2-1 Fractionation sceme of the DCM extract of G. pfeifferi; n. e. not examined; chromatographic conditions see section 1 . 


\section{Spectra of Isolated Compounds}

In the following, the spectra used for the identification of the isolated compounds are collected. Interesting publications concerning the compounds are summarized in table form.

For structure elucidation, at first EI- and HR-MS spectra as well as ${ }^{1} \mathrm{H}$ - and ${ }^{13} \mathrm{C}-\mathrm{NMR}$ spectra were obtained. If a compound could be unambiguously assigned to a structure already described using these data, no further structure analysis was done. The UV-vis spectra shown for the compounds are those obtained by HPLC using a diode array detector and are therefore recorded in an undefined solvent in an unknown concentration. 


\subsection{Ergosta-7,22-diene-3-one}

Isolation see Figure 2-1 on page 2. white flakes (acetone). yield $37.3 \mathrm{mg}$ (min. $0.0172 \%$ in dried fruiting bodies of G. pfeifferi). TLC: $\mathrm{R}_{\mathrm{f}} 0.85$; very weak fluorescence quenching at $254 \mathrm{~nm}$, no fluorescence at $366 \mathrm{~nm}$; orange with anisaldehyde-sulphuric acid reagent.

HRFTICRMS [M+H] ${ }^{+}$: calcd for $\mathrm{C}_{28} \mathrm{H}_{45} \mathrm{O}$ : 397.3470; found: 397.3462 .

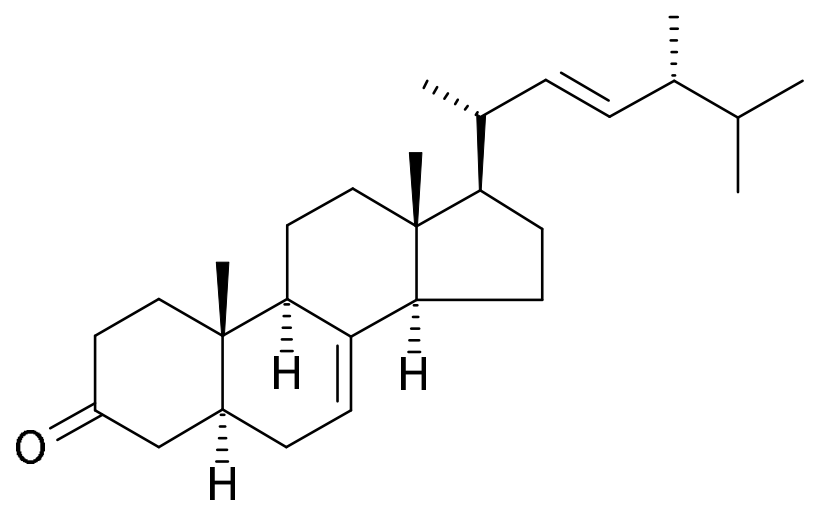

Ergosta-7,22-dien-3-one

\subsubsection{El-MS}

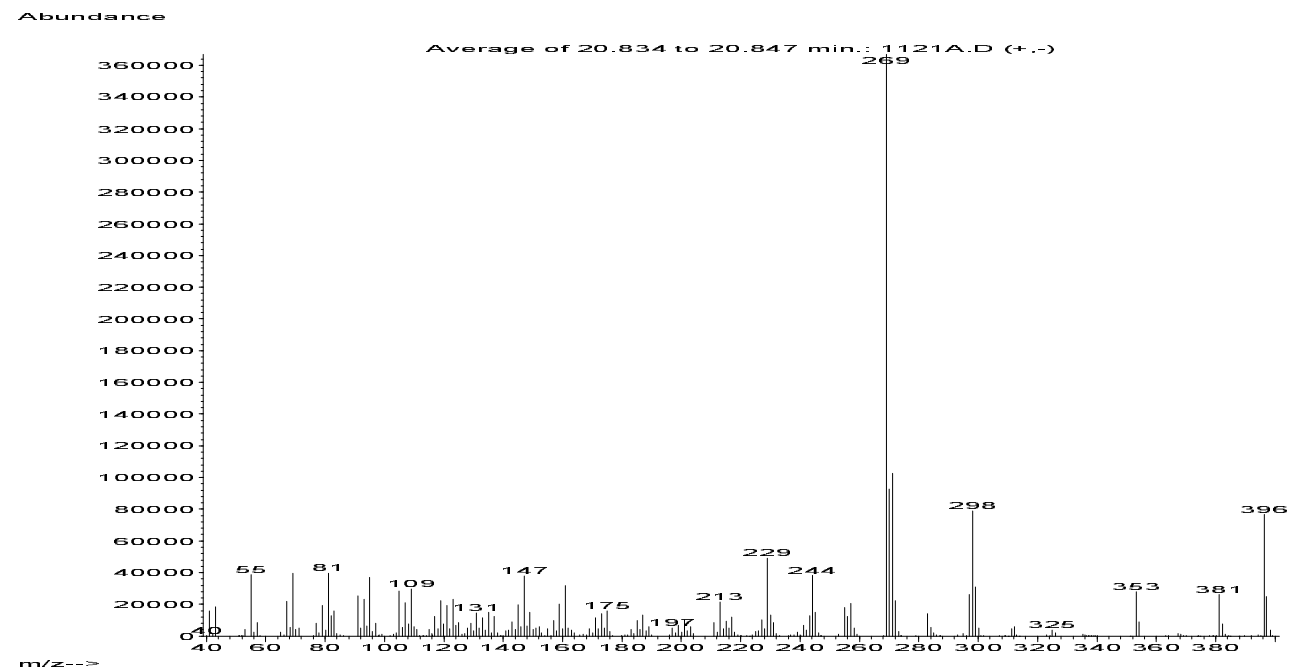

Figure 3-1 EI-MS Ergosta-7,22-dien-3-one 


\subsection{2 ${ }^{1} \mathrm{H}-\mathrm{NMR}$}

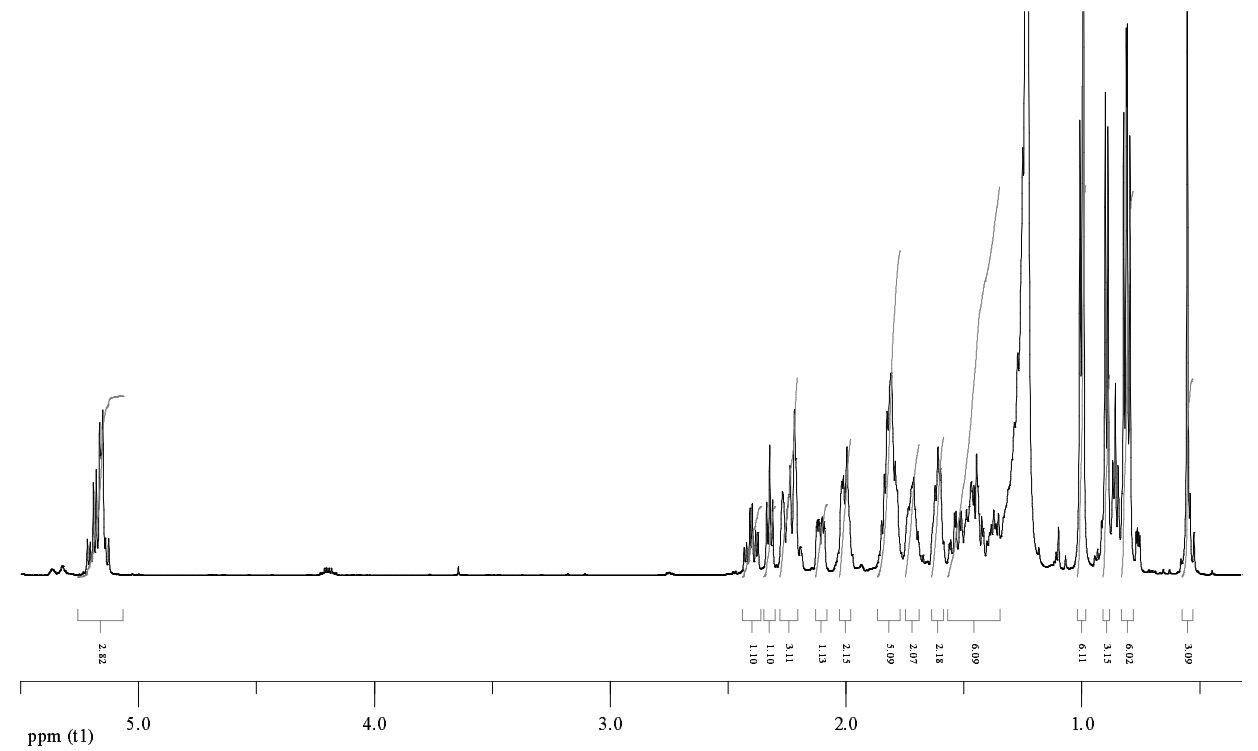

Figure 3-2 ${ }^{1} \mathrm{H}-\mathrm{NMR}$ Ergosta-7,22-dien-3-one in $\mathrm{CDCl}_{3}$

\subsection{3 ${ }^{13} \mathrm{C}-\mathrm{NMR}$}

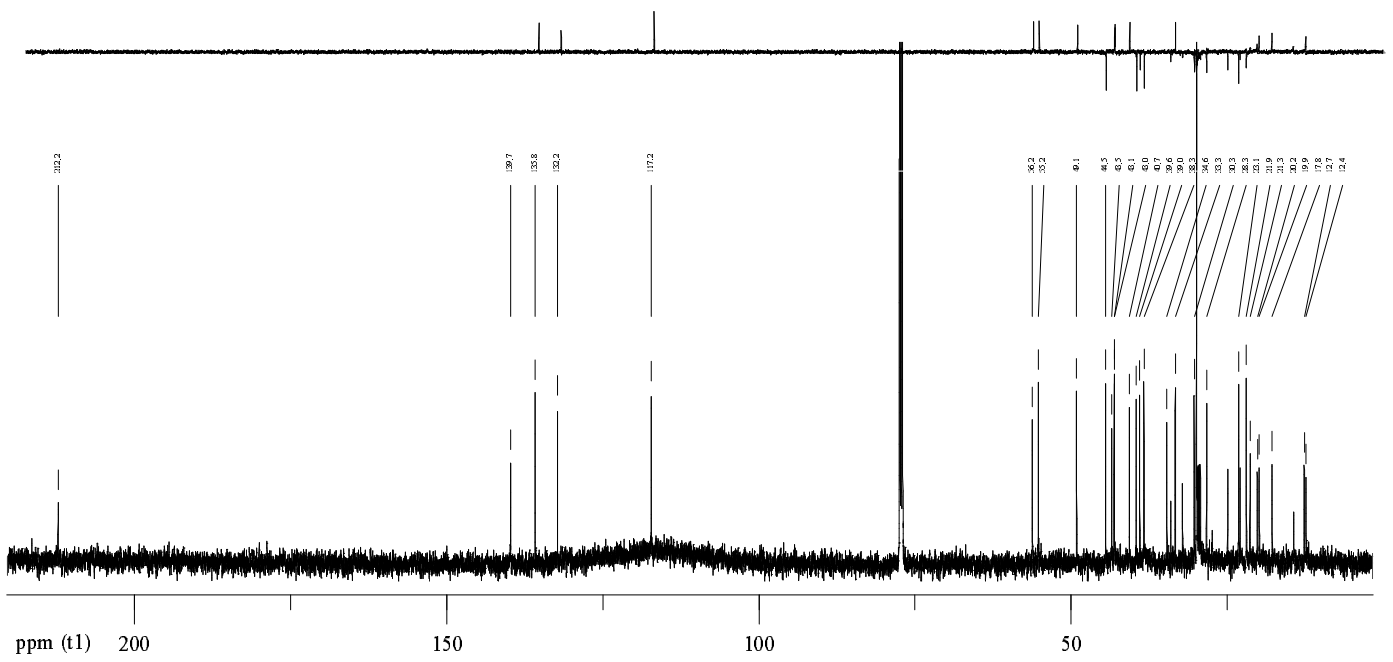

Figure 3-3 ${ }^{13} \mathrm{C}-\mathrm{NMR}$ and DEPT135 Ergosta-7,22-dien-3-one in $\mathrm{CDCl}_{3}$ 


\subsubsection{Literature}

\begin{tabular}{|c|c|c|c|c|c|}
\hline year & isolated from & yield $(\%)$ & structure analysis & biol. activity & in \\
\hline 1975 & G. applanatum & 0.0036 & $\begin{array}{l}\operatorname{mp},[\alpha]_{\mathrm{D}}, \mathrm{IR}, \mathrm{R}_{\mathrm{f}}, \mathrm{EI}- \\
\mathrm{MS}\end{array}$ & $\mathrm{n} / \mathrm{a}$ & 1 \\
\hline 1984 & G. australe & 0.0063 & $\begin{array}{l}\mathrm{mp}, \quad \mathrm{IR}, \quad \mathrm{R}_{\mathrm{f}}, \quad \mathrm{EI}-\mathrm{MS}, \\
\mathrm{UV}-\mathrm{vis},{ }^{1} \mathrm{H}-\mathrm{NMR}\end{array}$ & $\mathrm{n} / \mathrm{a}$ & 2 \\
\hline 1980 & G. applanatum & 0.0468 & $\begin{array}{l}\operatorname{mp}, \quad[\alpha]_{\mathrm{D}},{ }^{1} \mathrm{H}-,{ }^{13} \mathrm{C}- \\
\mathrm{NMR}\end{array}$ & $\mathrm{n} / \mathrm{a}$ & 3 \\
\hline 1985 & Polyporus umbellatus & 0.0073 & $\begin{array}{l}\operatorname{mp},[\alpha]_{\mathrm{D}}, \mathrm{IR}, \mathrm{EI}-\mathrm{MS} \\
{ }^{1} \mathrm{H}-\mathrm{NMR}\end{array}$ & $\begin{array}{lr}\text { weak inhibitory } & \text { effect } \\
\text { on ADP-induced rabbit } \\
\text { platelet aggregation }\end{array}$ & 4 \\
\hline 1989 & G. lucidum & $\mathrm{n} / \mathrm{a}$ & $\mathrm{n} / \mathrm{a}$ & $\mathrm{n} / \mathrm{a}$ & 5 \\
\hline 1998 & G. applanatum & 0.0009 & $\mathrm{n} / \mathrm{a}$ & $\mathrm{n} / \mathrm{a}$ & 6 \\
\hline 1998 & G. neo-japonicum & 0.0055 & $\mathrm{n} / \mathrm{a}$ & $\mathrm{n} / \mathrm{a}$ & 6 \\
\hline 2002 & G. concinna & 0.0588 & $\mathrm{n} / \mathrm{a}$ & $\mathrm{n} / \mathrm{a}$ & 7 \\
\hline
\end{tabular}

Table 3-1 Literature Ergosta-7,22-dien-3-one 


\subsection{Ganoderal A}

Isolation see Figure 2-1 on page 2. white ceraceous plates (acetone). yield $29.9 \mathrm{mg}$ (min. $0.0138 \%$ in dried fruiting bodies of G. pfeifferi). TLC: $\mathrm{R}_{\mathrm{f}} 0.84$; fluorescence quenching at $254 \mathrm{~nm}$, no fluorescence at $366 \mathrm{~nm}$; orange-red with anisaldehyde-sulphuric acid reagent.

HRFTICRMS [M+H] ${ }^{+}$: calcd for $\mathrm{C}_{30} \mathrm{H}_{45} \mathrm{O}_{2}$ : 437.3420; found: 437.3419 .

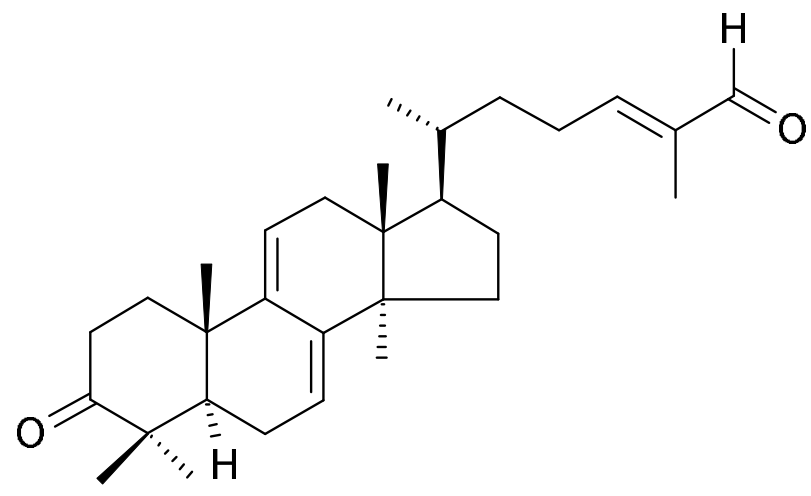

(6) Ganoderal A

\subsubsection{UV-vis}

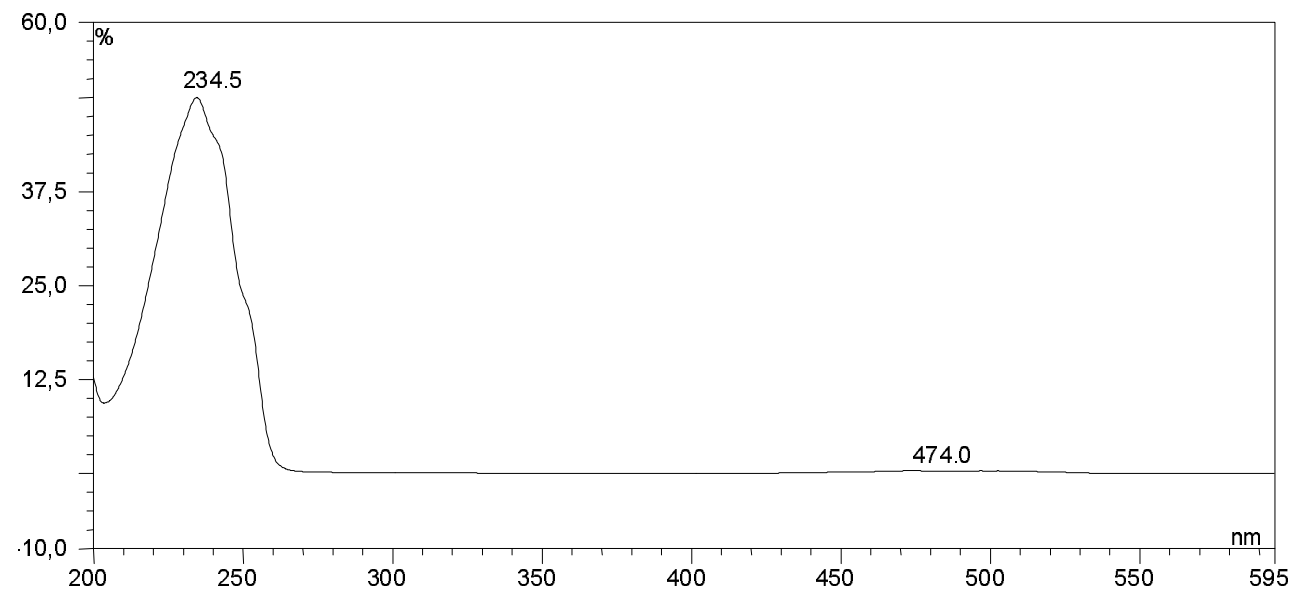

Figure 3-4 UV-vis Ganoderal A 


\subsubsection{EI-MS}

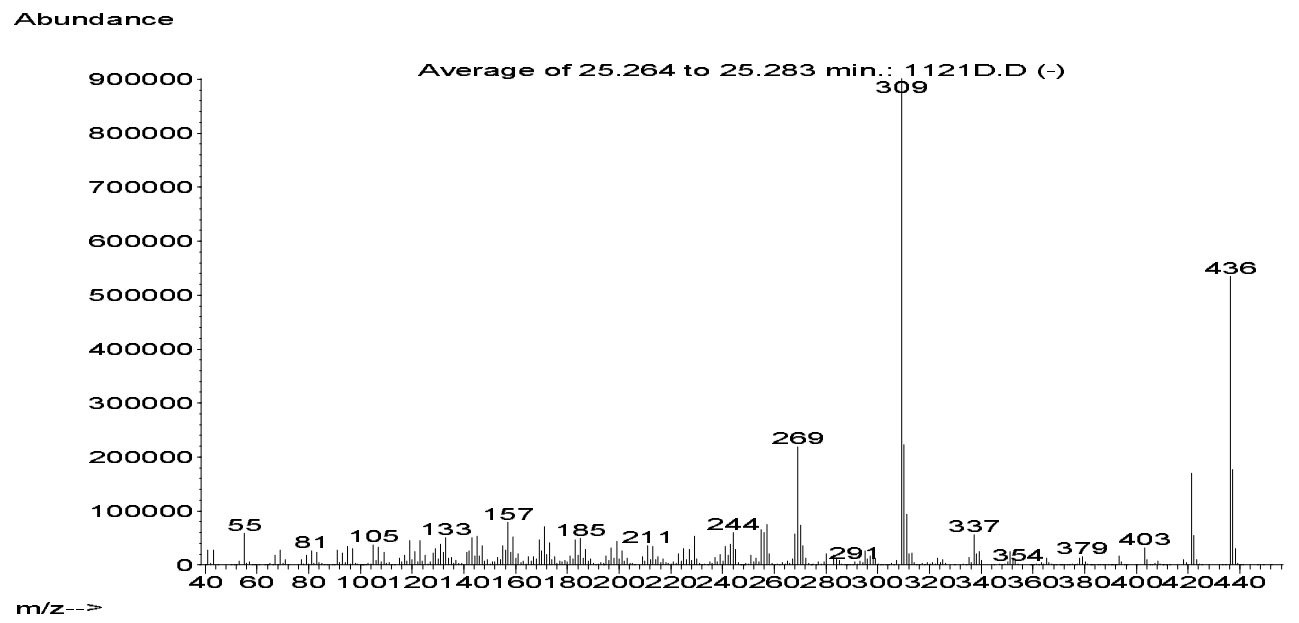

Figure 3-5 EI-MS Ganoderal A

\subsection{3 ${ }^{1} \mathrm{H}-\mathrm{NMR}$}

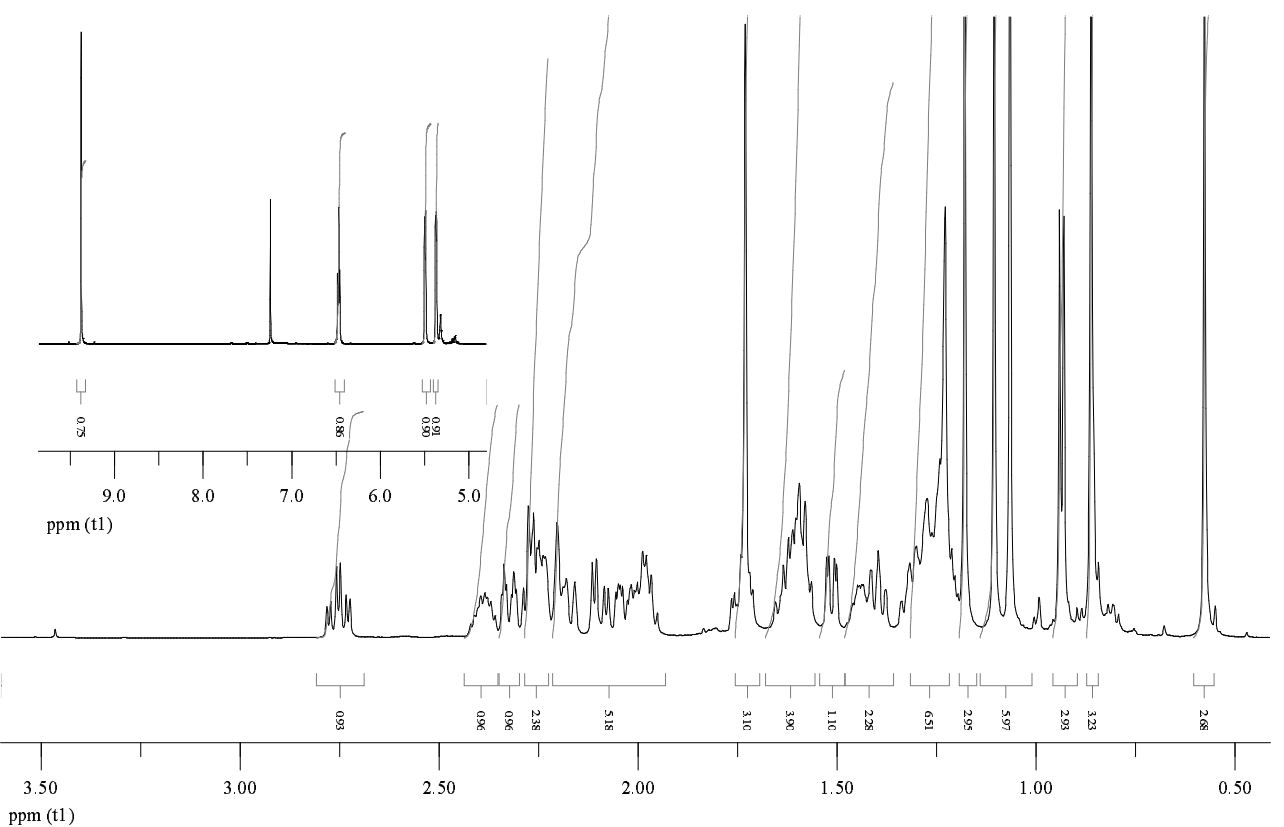

Figure 3-6 ${ }^{1} \mathrm{H}-\mathrm{NMR}$ Ganoderal $\mathrm{A}$ in $\mathrm{CDCl}_{3}$ 


\subsection{4 ${ }^{13} \mathrm{C}-\mathrm{NMR}$}

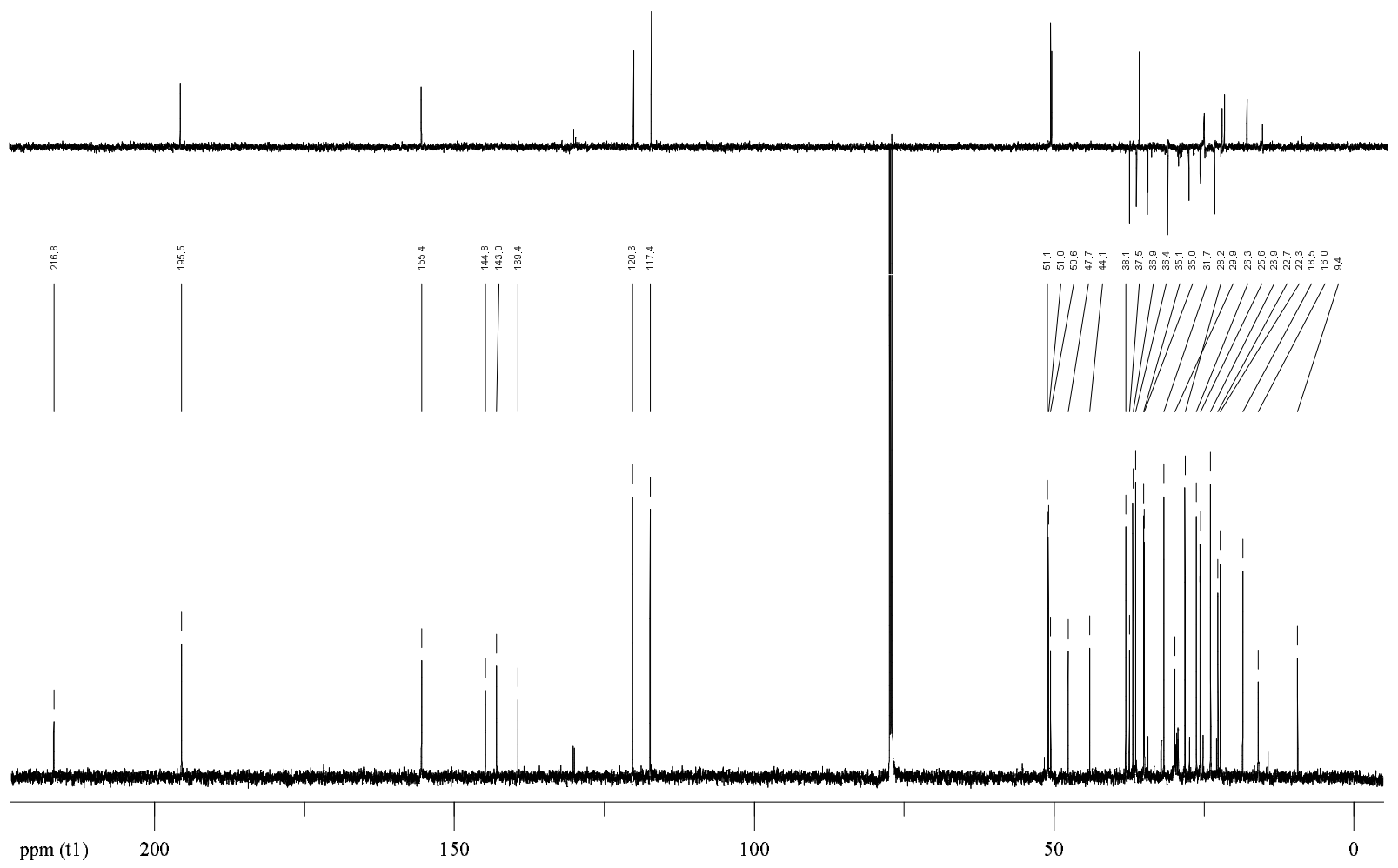

Figure 3-7 ${ }^{13} \mathrm{C}-\mathrm{NMR}$ and DEPT135 Ganoderal $\mathrm{A}$ in $\mathrm{CDCl}_{3}$

\subsubsection{Literature}

\begin{tabular}{|c|c|c|c|c|c|}
\hline year & isolated from & yield $(\%)$ & structure analysis & biol. activity & in \\
\hline 1986 & G. lucidum & $\mathrm{n} / \mathrm{a}$ & $\begin{array}{l}\mathrm{mp},[\alpha]_{\mathrm{D}}, \mathrm{UV}-\mathrm{vis}, \mathrm{R}_{\mathrm{f}} \\
\text { EI-MS, }{ }^{1} \mathrm{H}-\mathrm{NMR}\end{array}$ & $\begin{array}{l}\text { inhibition of angiotensin } \\
\text { converting enzyme } \\
\left(\mathrm{IC}_{50}>10^{-5} \mathrm{M}\right)\end{array}$ & 8 \\
\hline 1987 & G. lucidum & $\mathrm{n} / \mathrm{a}$ & $\begin{array}{l}\text { mp, EI-MS, IR, UV- } \\
\text { vis }\end{array}$ & $\mathrm{n} / \mathrm{a}$ & 9 \\
\hline 1998 & G. neo-japonicum & 0.0003 & $\mathrm{n} / \mathrm{a}$ & $\mathrm{n} / \mathrm{a}$ & 6 \\
\hline 2002 & G. concinna & 0.0035 & ${ }^{13} \mathrm{C}-\mathrm{NMR}$ & $\mathrm{n} / \mathrm{a}$ & 7 \\
\hline
\end{tabular}

Table 3-2 Literature Ganoderal A 


\subsection{Ergosta-4,6,8(14),22-tetraene-3-one}

Isolation see Figure. 2-1 on page 2. yellow amorphous powder. yield $4.7 \mathrm{mg}$ (min. $0.0016 \%$ in dried fruiting bodies of G. pfeifferi). TLC: $\mathrm{R}_{\mathrm{f}}$ 0.65; fluorescence quenching at $254 \mathrm{~nm}$, intense blue fluorescence at $366 \mathrm{~nm}$; yellow with anisaldehyde-sulphuric acid reagent.

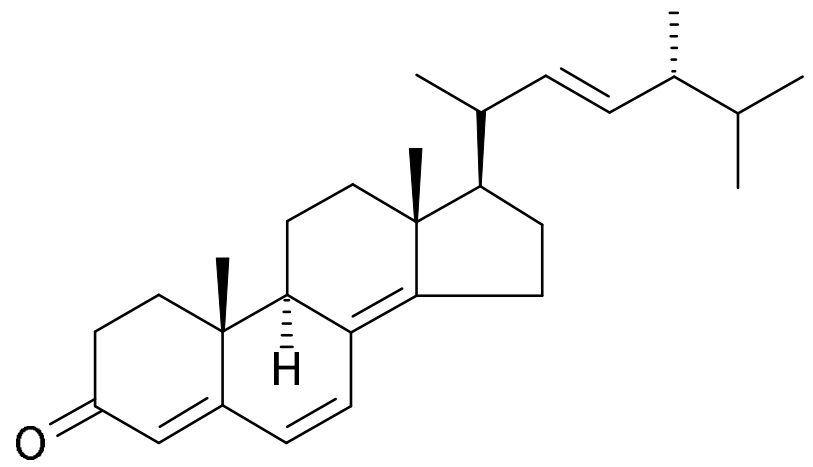

Ergosta-4,6,8(14),22-tetraen-3-one

\subsubsection{UV-vis}

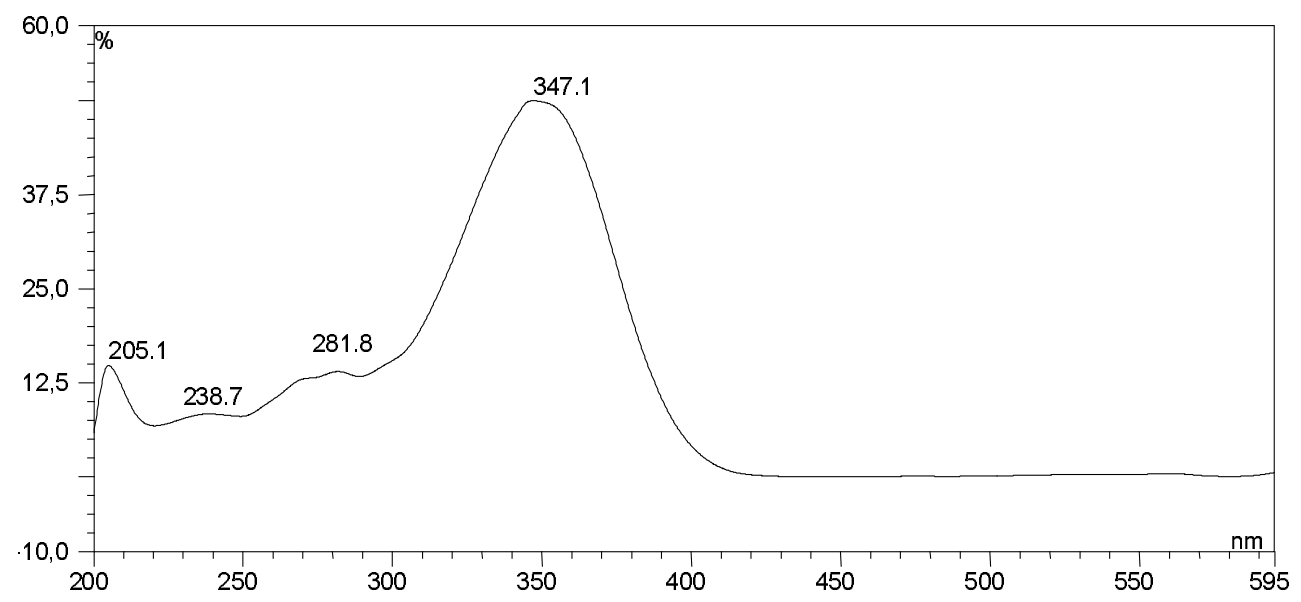

Figure 3-8 UV-vis Ergosta-4,6,8(14),22-tetraen-3-one 


\subsubsection{EI-MS}

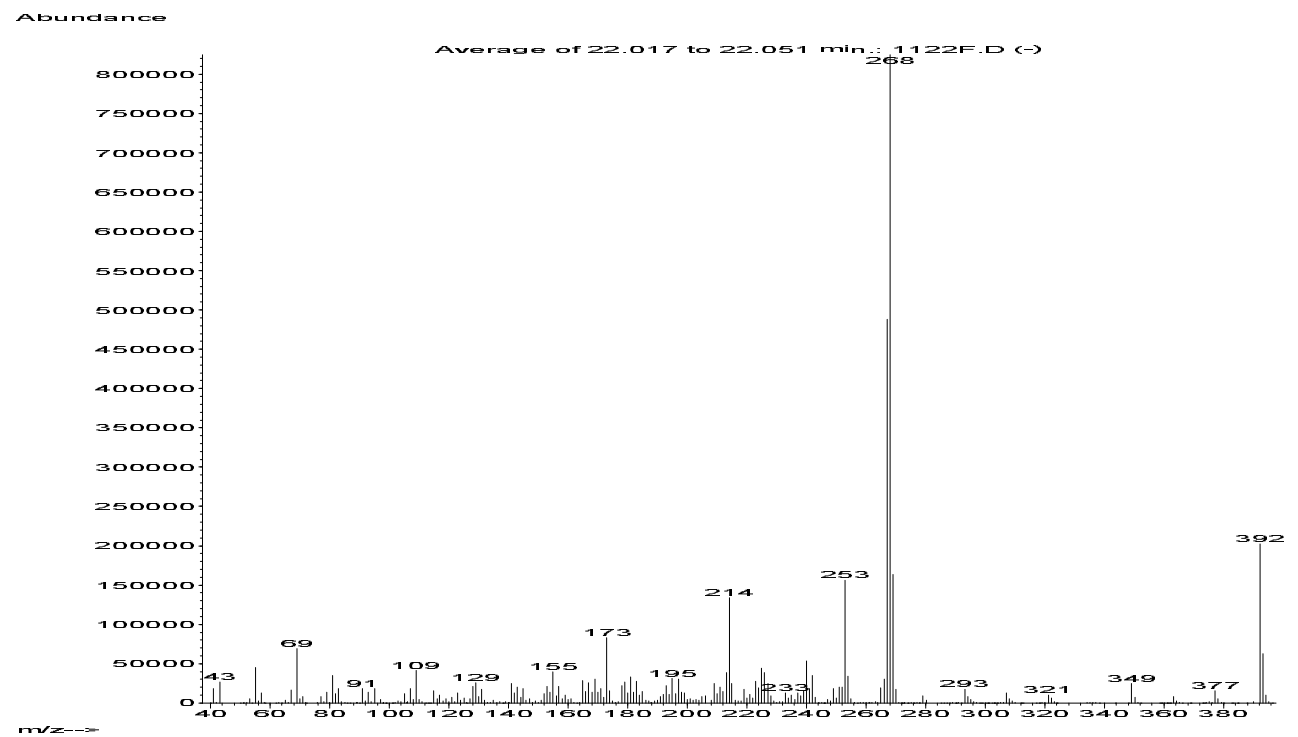

Figure 3-9 EI-MS Ergosta-4,6,8(14),22-tetraen-3-one

\subsection{3 ${ }^{1} \mathrm{H}-\mathrm{NMR}$}

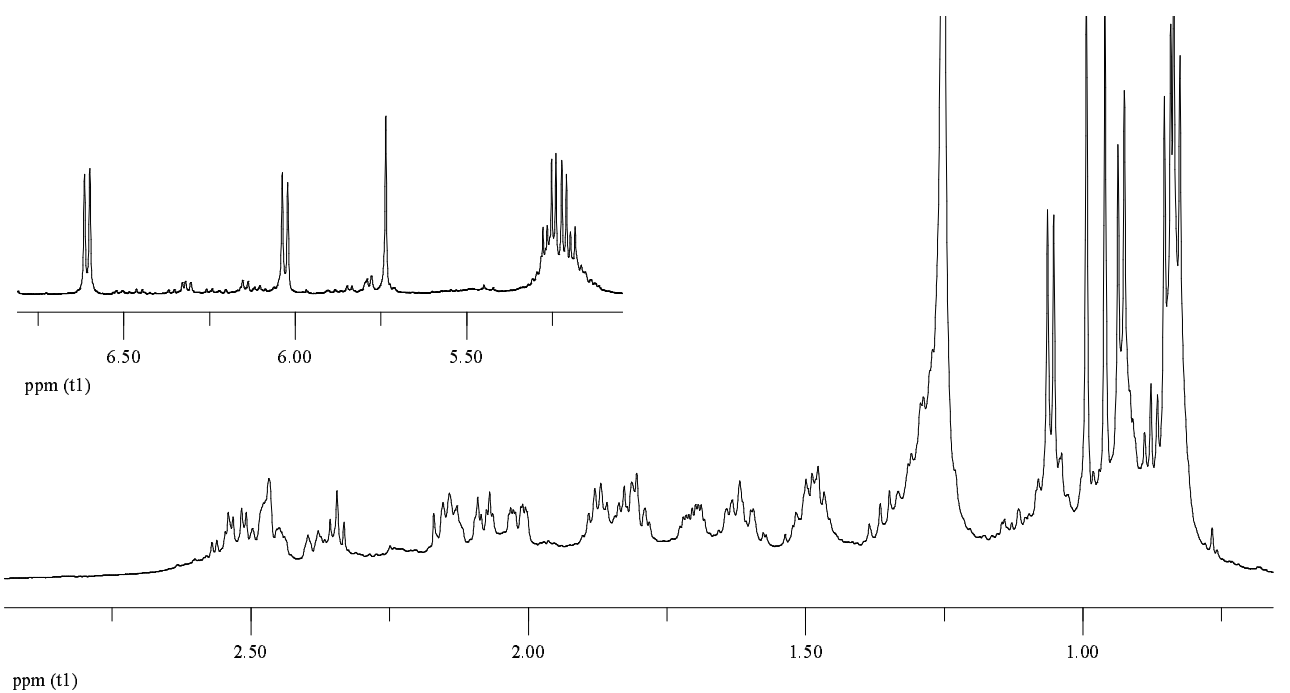

Figure 3-10 $\quad{ }^{1} \mathrm{H}-\mathrm{NMR}$ Ergosta-4,6,8(14),22-tetraen-3-on in $\mathrm{CDCl}_{3}$ 


\subsection{4 ${ }^{13} \mathrm{C}-\mathrm{NMR}$}

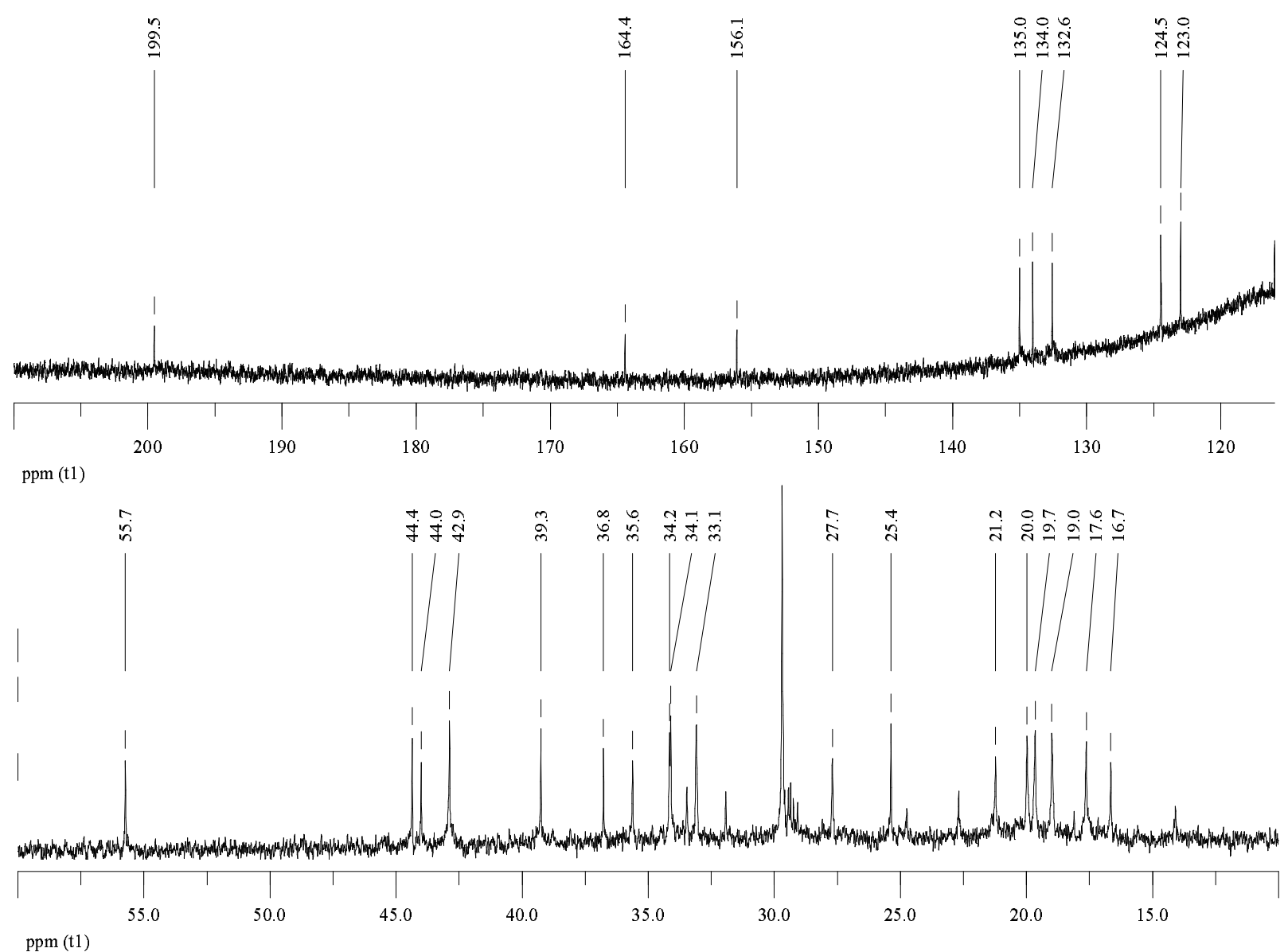

Figure 3-11 ${ }^{13} \mathrm{C}-\mathrm{NMR}$ of Ergosta-4,6,8(14),22-tetraen-3-on in $\mathrm{CDCl}_{3}$ 


\subsubsection{Literature}

\begin{tabular}{|c|c|c|c|c|c|}
\hline year & isolated from & yield (\%) & structure analysis & biol. activity & in \\
\hline 1967 & Candida utilis & 0.0002 & $\begin{array}{l}\mathrm{R}_{\mathrm{f}}, \mathrm{mp},[\alpha]_{\mathrm{D}}, \mathrm{UV}, \mathrm{IR}, \\
\text { EI-MS, }{ }^{1} \mathrm{H}-\mathrm{NMR}\end{array}$ & $\mathrm{n} / \mathrm{a}$ & 10 \\
\hline 1968 & Fomes officinalis & $\mathrm{n} / \mathrm{a}$ & $\begin{array}{l}\text { UV, IR, }[\alpha]_{\mathrm{D}}, \text { EI-MS, } \\
{ }^{1} \mathrm{H}-\mathrm{NMR}\end{array}$ & $\mathrm{n} / \mathrm{a}$ & 11 \\
\hline 1998 & G. applanatum & 0.0001 & ${ }^{1} \mathrm{H}-,{ }^{13} \mathrm{C}-\mathrm{NMR}$ & $\mathrm{n} / \mathrm{a}$ & 6 \\
\hline 2004 & synthesis & approx. 10 & $\mathrm{n} / \mathrm{a}$ & $\begin{array}{l}\text { aldosterone antagonist with- } \\
\text { out a direct effect on } \mathrm{Na} / \mathrm{K} \\
\text { ion channels in Wistar rats } \\
\rightarrow \text { diuretic effect; bioavail- } \\
\text { able after oral admission }\end{array}$ & 12 \\
\hline 2002 & Paecilomyces spec. & 0.0005 & $\begin{array}{l}\text { mp, UV, EI-MS, }{ }^{1} \mathrm{H}- \\
{ }^{13} \mathrm{C}-\mathrm{NMR}\end{array}$ & $\begin{array}{l}\text { moderate cytotoxicity } \\
\text { agains five tumor cell lines } \\
\left(\mathrm{EC}_{50} 23-35 \mathrm{mM}\right)\end{array}$ & 13 \\
\hline 2002 & Grifola frondosa & 0.0013 & ${ }^{1} \mathrm{H}-\mathrm{NMR}$ & $\begin{array}{l}\text { inhibitory activity on COX- } \\
1 \quad(55 \% \text { inhibition at } 250 \\
\mu \mathrm{g} / \mathrm{mL}) \text { and COX-2 }(70 \% \\
\text { inhibition at } 250 \mu \mathrm{g} / \mathrm{mL}) \\
\text { antioxidative effect }(42 \% \text { in- } \\
\text { hibition of liposome peroxi- } \\
\text { dation at } 100 \mu \mathrm{g} / \mathrm{mL})\end{array}$ & 14 \\
\hline
\end{tabular}

Table 3-3 Literature Ergosta-4,6,8(14),22-tetraen-3-one

Further interesting literature

- Biosynthetic studies in Penicillium rubrum ${ }^{15}$

- Potential use of the fluorescence of ergosta-4,6,8(14),22-tetraen-3-one as marker compound in the standardization of the chinese drug Polyporus Sclerotion ${ }^{16}$ or as chemical marker for past or presently occurring fungal infections of wheat flour ${ }^{17}$ or tobacco ${ }^{18}$

- studies on the photochemical reactions of ergosta-4,6,8(14),22-tetraen-3-on on irradiation with UV light ${ }^{19}$

- Isolation from the sponge Dysidea herbacea ${ }^{20}$ and some other Ganoderma species like G. lucidum $^{21,22}$ and G. neo-japonicum ${ }^{6}$ 


\subsection{Ergosta-7,22-diene-3 $\beta$-ol}

Isolation see Figure 2-1 on page 2. white, woolen needles. yield $18.8 \mathrm{mg}$ (min. $0.0086 \%$ in dried fruiting bodies of G. pfeifferi). TLC: $\mathrm{R}_{\mathrm{f}} 0.54$; very weak fluorescence quenching at $254 \mathrm{~nm}$, no fluorescence at $366 \mathrm{~nm}$; gray-violet with anisaldehyde-sulphuric acid reagent.

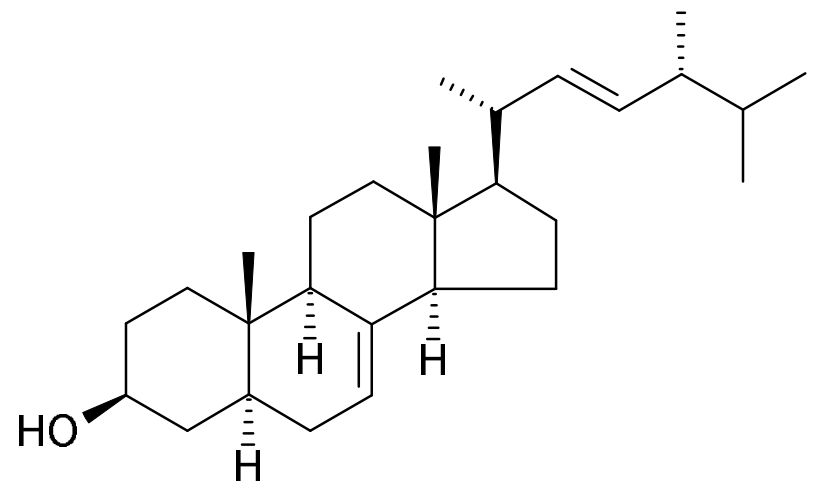

(4) Ergosta-7,22-dien-3 $\beta$-ol

\subsubsection{EI-MS}

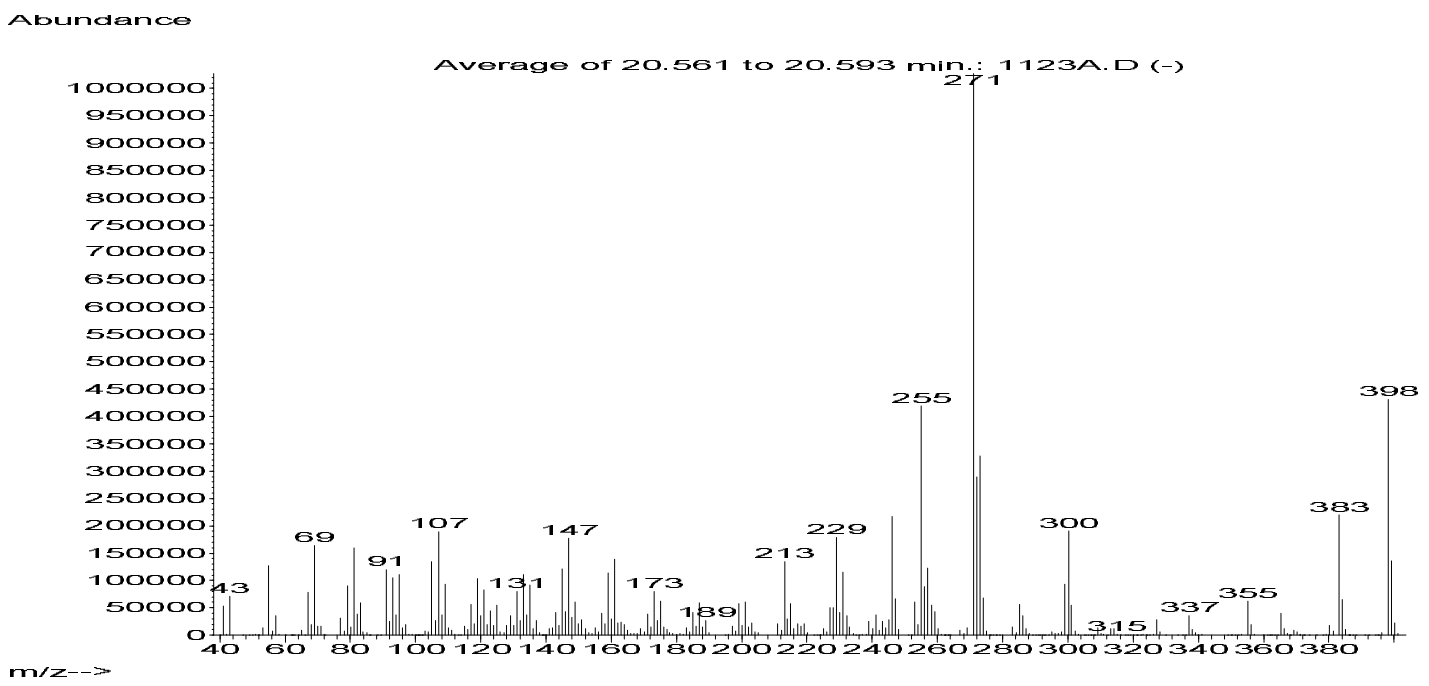

Figure 3-12 EI-MS Ergosta-7,22-dien-3 $\beta$-ol 


\subsection{2 ${ }^{1} \mathrm{H}-\mathrm{NMR}$}

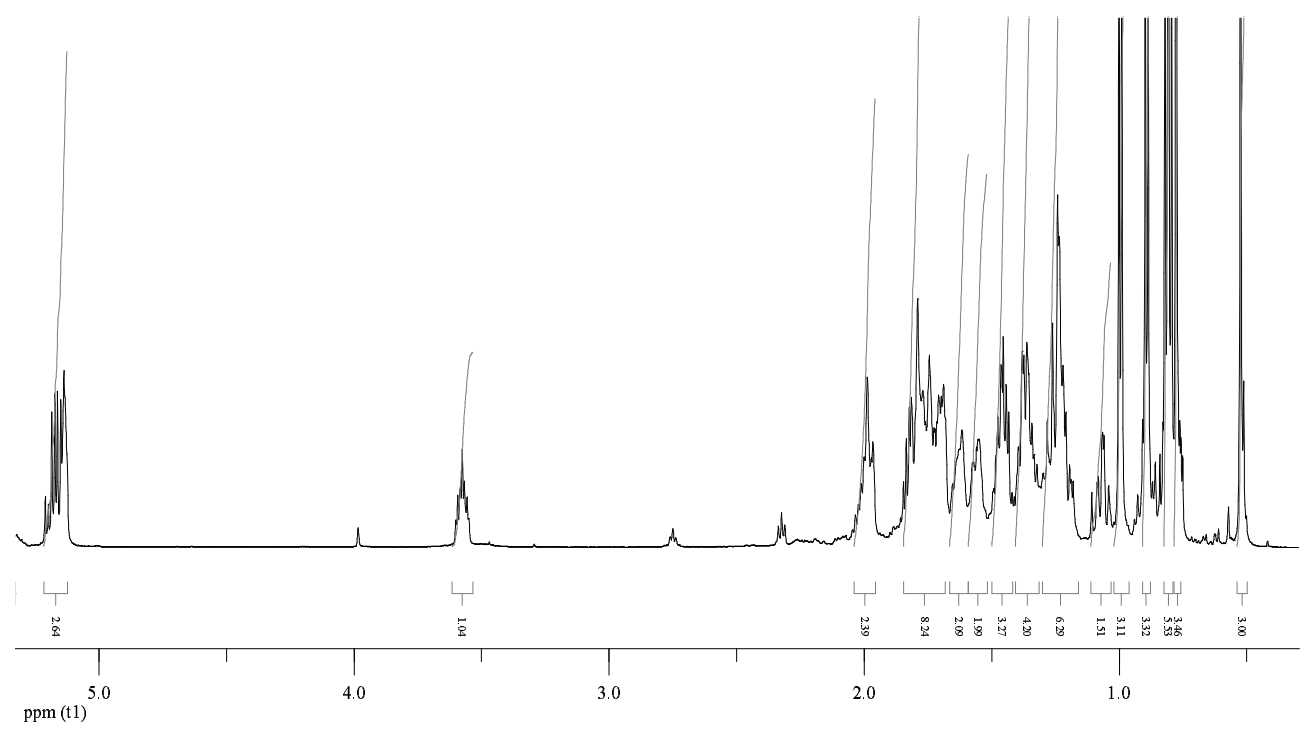

Figure 3-13 ${ }^{1} \mathrm{H}-\mathrm{NMR}$ Ergosta-7,22-dien- $3 \beta$-ol in $\mathrm{CDCl}_{3}$

\subsection{3 ${ }^{13} \mathrm{C}-\mathrm{NMR}$}

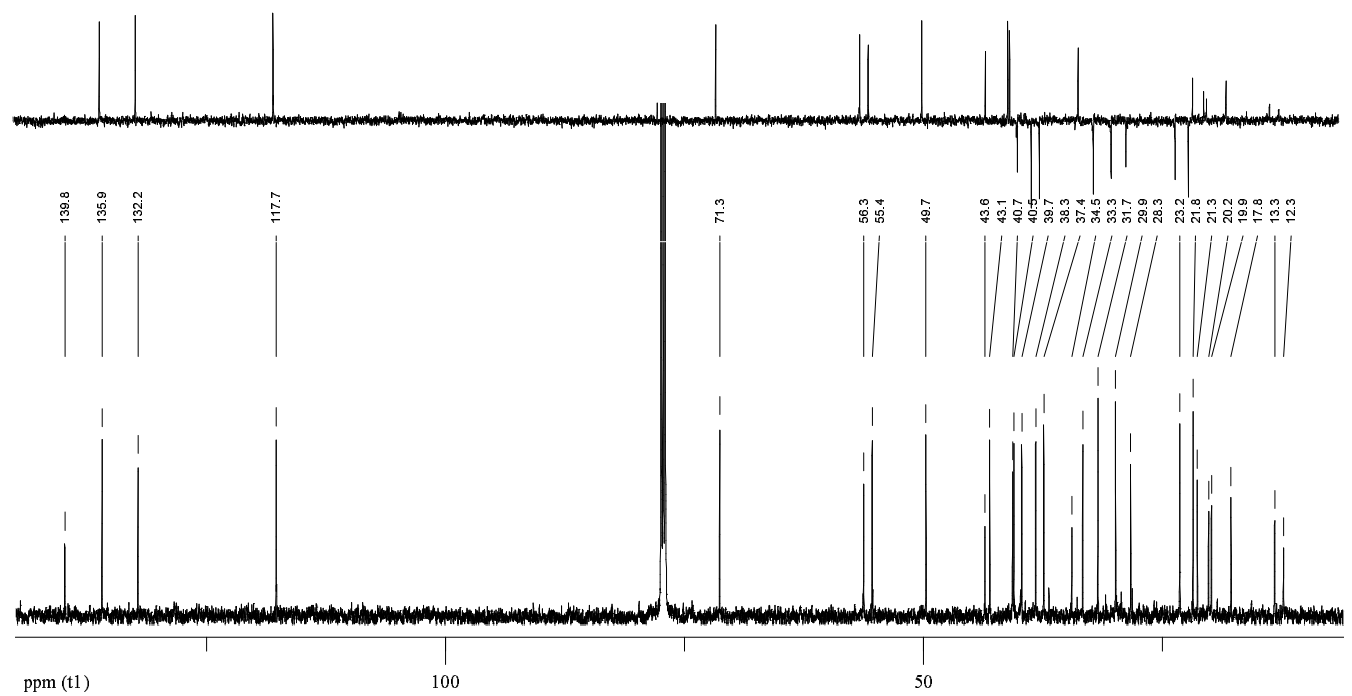

Figure 3-14 ${ }^{13} \mathrm{C}-\mathrm{NMR}$ and DEPT135 of Ergosta-7,22-dien-3 $\beta$-ol in $\mathrm{CDCl}_{3}$ 


\subsubsection{Literature}

Ergosta-7,22-dien-3 $\beta$-ol has been found in many fungi. Therefore, only a small number of interesting references is given here.

\begin{tabular}{|c|c|c|c|c|c|}
\hline year & isolated from & yield $(\%)$ & structure analysis & biol. activity & in \\
\hline 1975 & G. applanatum & 0.0492 & $\begin{array}{l}\operatorname{mp},[\alpha]_{\mathrm{D}}, \mathrm{R}_{\mathrm{f}}, \mathrm{IR}, \\
\text { EI-MS }\end{array}$ & $\mathrm{n} / \mathrm{a}$ & 1 \\
\hline 1985 & Polyporus umbellatus & 0.013 & $\begin{array}{l}\operatorname{mp},[\alpha]_{\mathrm{D}}, \mathrm{IR}, \mathrm{EI}- \\
\mathrm{MS},{ }^{1} \mathrm{H}-\mathrm{NMR}\end{array}$ & $\begin{array}{l}\text { enhancing effect on } \\
\text { collagen- and ADP-induced } \\
\text { rabbit platelet aggregation }\end{array}$ & 4 \\
\hline 1987 & Inonotus obliquus & $\mathrm{n} / \mathrm{a}$ & $\mathrm{n} / \mathrm{a}$ & $\begin{array}{l}\text { slightly active or inactive } \\
\text { against Walker } 256 \text { cells, } \\
\text { possibly active against } \\
\text { MCF-7 cells }\end{array}$ & 23,24 \\
\hline 1996 & Fomitopsis pinicola & 0.0075 & $\begin{array}{l}\operatorname{mp},[\alpha]_{\mathrm{D}}, \mathrm{R}_{\mathrm{f}},{ }^{1} \mathrm{H}- \\
{ }^{13} \mathrm{C}-\mathrm{NMR}, \mathrm{EI}-\mathrm{MS}\end{array}$ & $\begin{array}{l}\text { no antibacterial activity } \\
\text { against } B \text {. subtilis }\end{array}$ & 25 \\
\hline 1998 & Phycomyces blakesleeanus & 0.03 & EI-MS, ${ }^{13} \mathrm{C}-\mathrm{NMR}$ & $\mathrm{n} / \mathrm{a}$ & 26 \\
\hline 1999 & G. applanatum & 0.14 & $\mathrm{n} / \mathrm{a}$ & $\begin{array}{l}\text { antibacterial activity (MIC } \\
0.125-2.0 \mathrm{mg} / \mathrm{mL} \text {; testet } \\
\text { against a number of gram- } \\
\text { positive and gram-negative } \\
\text { bacteria) }\end{array}$ & 27 \\
\hline 2003 & G. annulare & $\mathrm{n} / \mathrm{a}$ & $\mathrm{n} / \mathrm{a}$ & $\begin{array}{l}\text { no antifungal activity } \\
\text { against } M \text {. cannis and } T \text {. } \\
\text { mentagrophytes }\end{array}$ & 28 \\
\hline
\end{tabular}

Table 3-4 Literature Ergosta-7,22-dien-3 $\beta$-ol

Further interesting literature:

- Synthesis ${ }^{26}$

- Occurrence of (4) in Ganodermataceae: G. applanatum $(0.03 \%-0.05 \%))^{1,3,6,29}$ G. australe $(0.0017 \%),{ }^{30-32}$ G. carnosum $(0.0512 \%),{ }^{33}$ G. lucidum (0.0075), ${ }^{5,29,34}$ G. neojaponicum $(0.009 \%){ }^{6}$ G. tsugae $(\mathrm{n} / \mathrm{a})^{35}$ 


\subsection{Lucialdehyde D*}

Isolation see Figure 2-1 on page 2. colourless oil. yield $6.5 \mathrm{mg}$ (min. $0.003 \%$ in dried fruiting bodies of $G$. pfeifferi). TLC: $\mathrm{R}_{\mathrm{f}} 0.65$; fluorescence quenching at $254 \mathrm{~nm}$, no fluorescence at 366 nm; no colorization with anisaldehyde-sulphuric acid reagent.

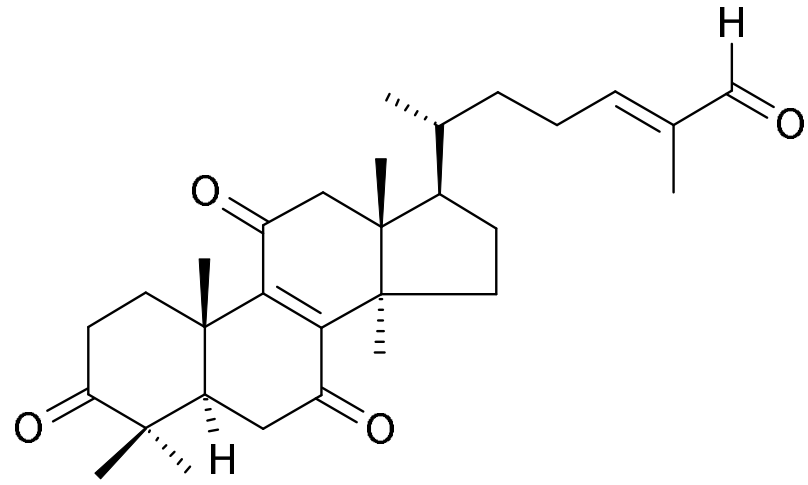

(1) Lucialdehyde D

\subsubsection{UV-vis}

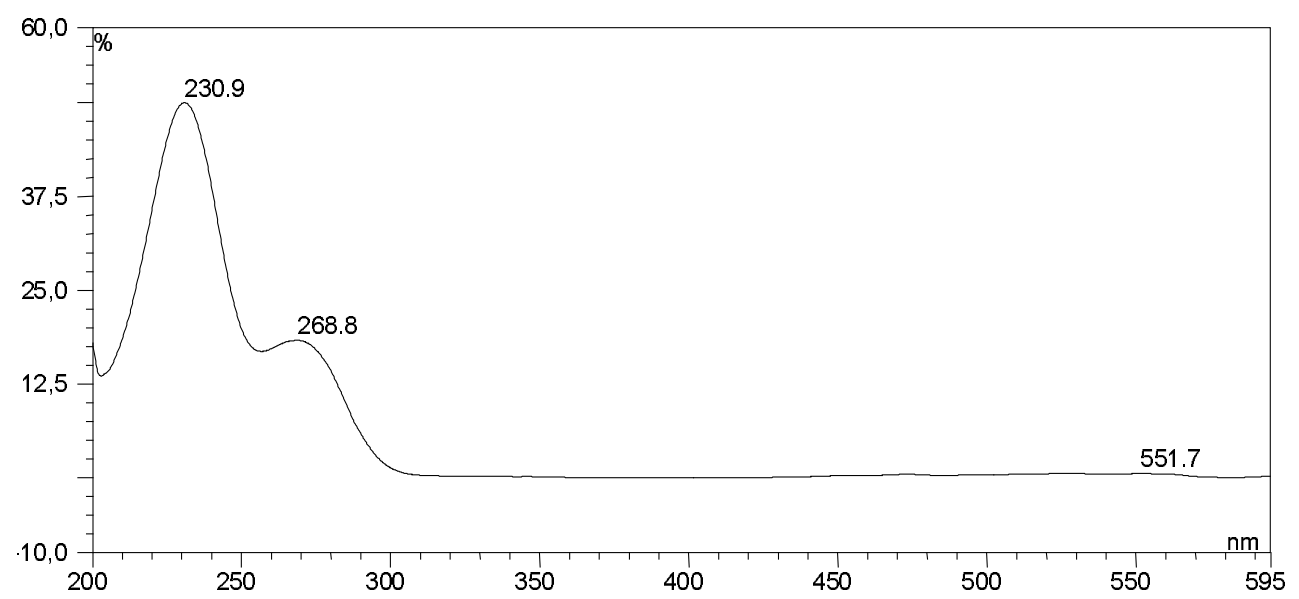

Figure 3-15 UV-vis Lucialdehyde D 


\subsubsection{El-MS}

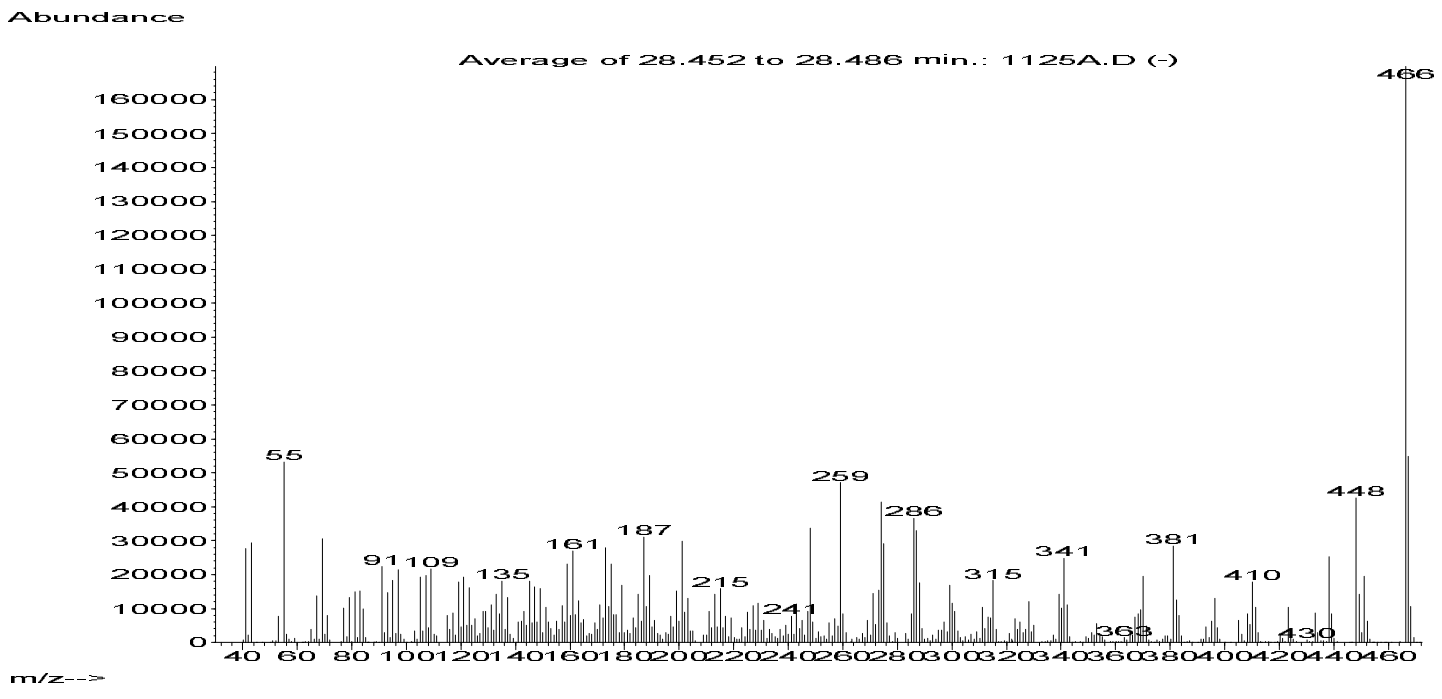

Figure 3-16 EI-MS Lucialdehyde D

\subsubsection{IR}

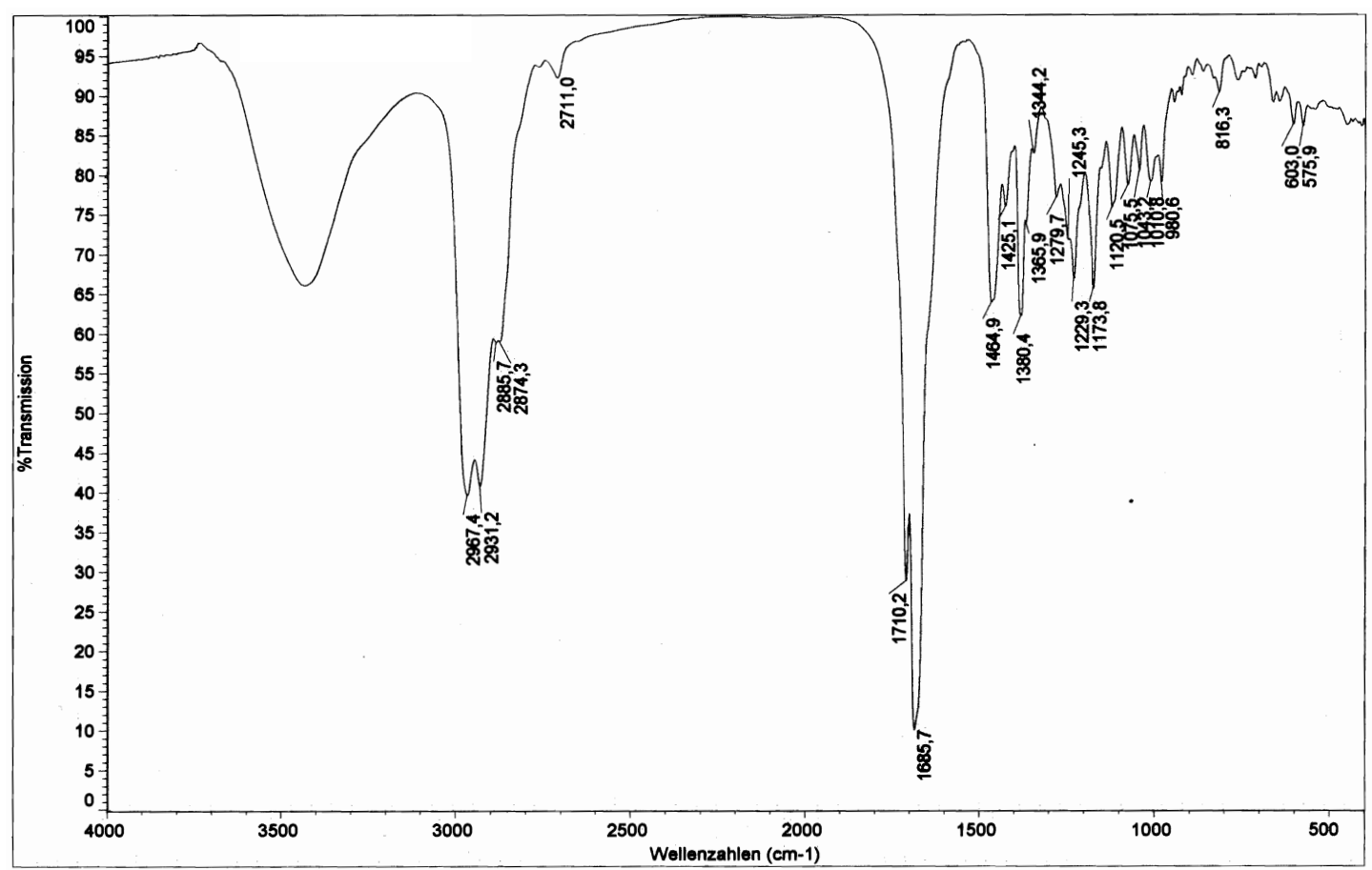

Figure 3-17 IR Lucialdehyde D 


\subsection{4 ${ }^{1} \mathrm{H}-\mathrm{NMR}$}

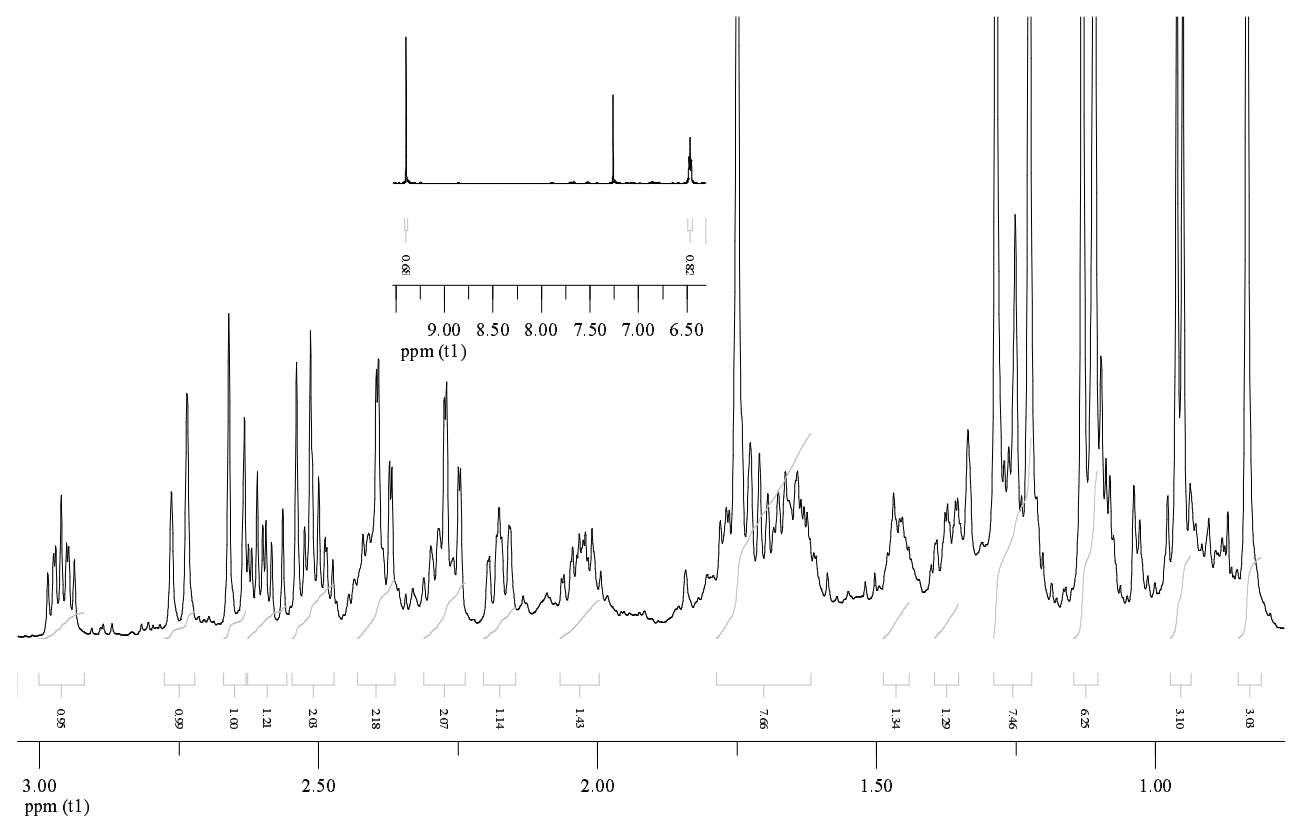

Figure 3-18 ${ }^{1} \mathrm{H}$-NMR Lucialdehyde D in $\mathrm{CDCl}_{3}$ 


\subsection{5 ${ }^{13}$ C-NMR}

\subsubsection{1 ${ }^{13} \mathrm{C}-\mathrm{NMR}$ overview}

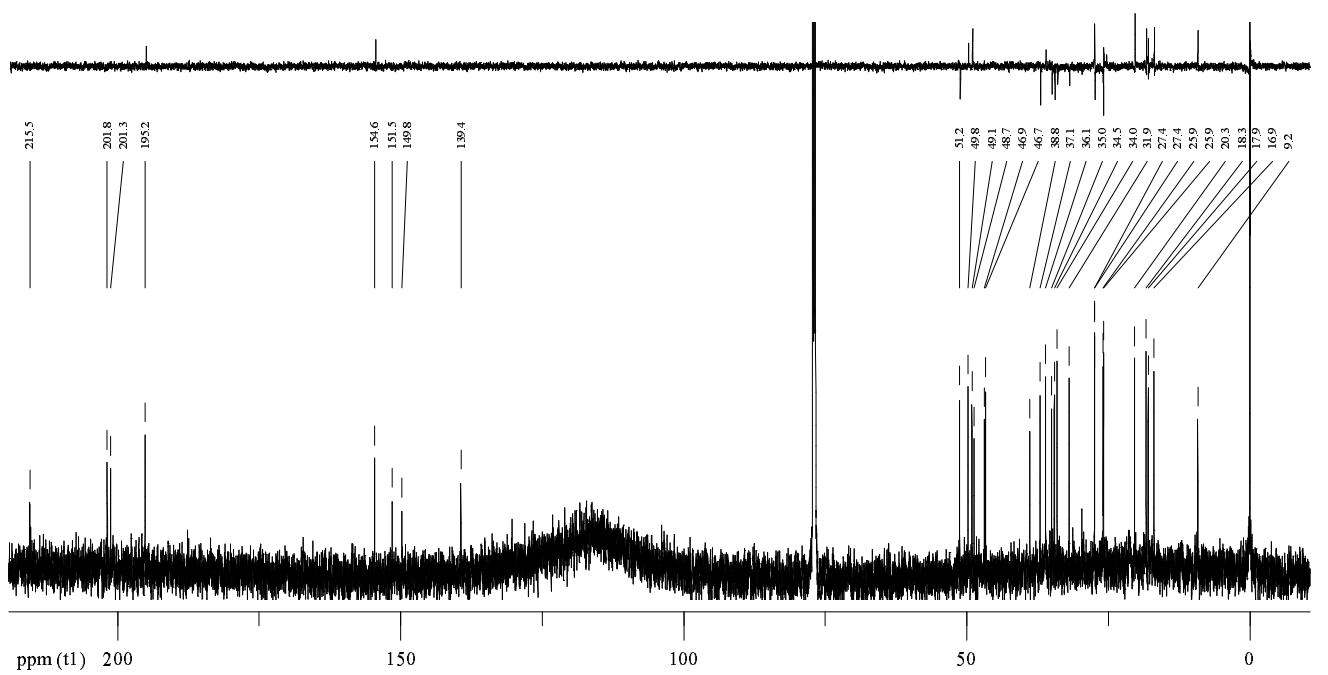

Figure 3-19 ${ }^{13} \mathrm{C}-\mathrm{NMR}$ and DEPT135 Lucialdehyde $\mathrm{D}$ in $\mathrm{CDCl}_{3}$ (overview)

\subsubsection{2 ${ }^{13}$ C-NMR detail}
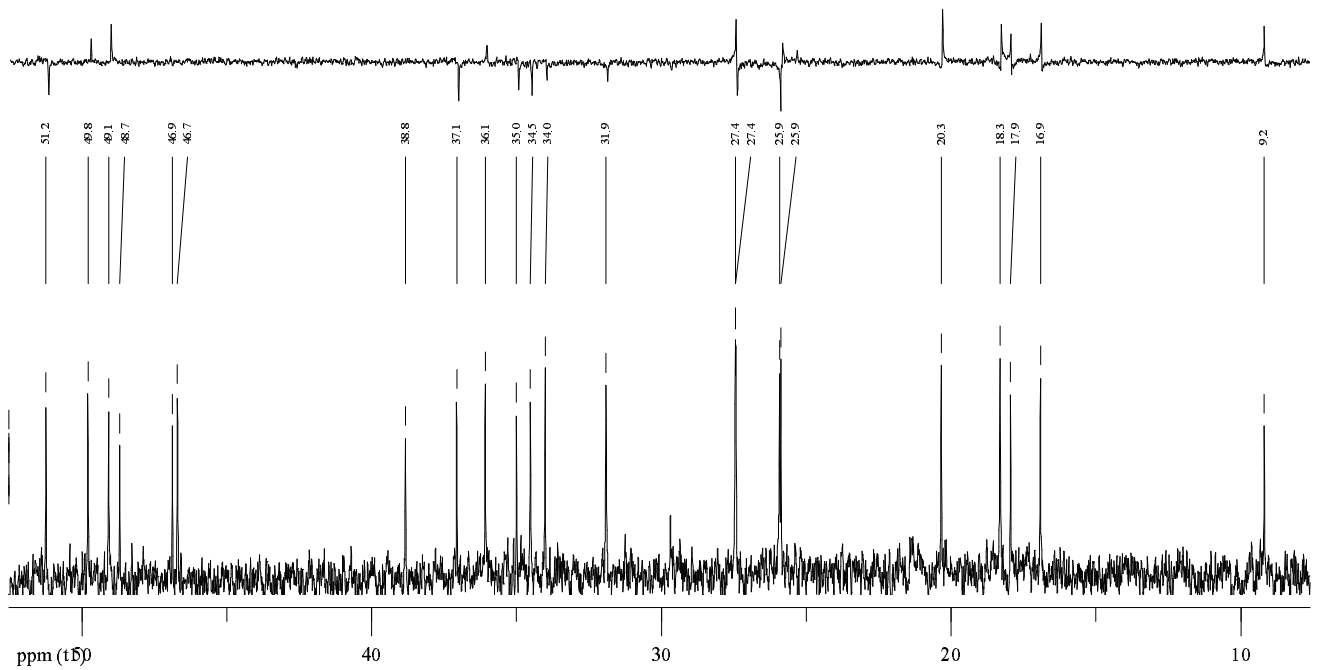

Figure 3-20 ${ }^{13} \mathrm{C}-\mathrm{NMR}$ and DEPT135 Lucialdehyde D in $\mathrm{CDCl}_{3}$ (7 to $53 \mathrm{ppm}$ ) 


\subsubsection{HSQC}

\subsubsection{HSQC overview}

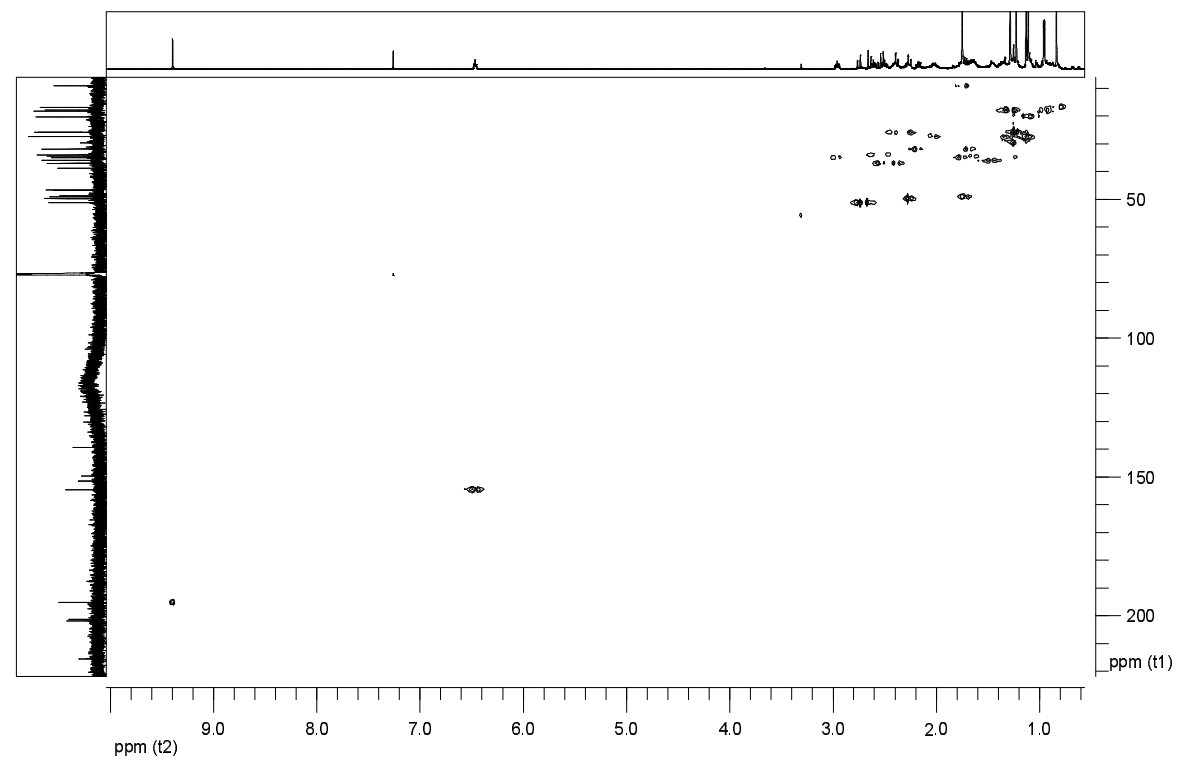

Figure 3-21 HSQC Lucialdehyde $\mathrm{D}$ in $\mathrm{CDCl}_{3}$ (overview)

\subsubsection{HSQC detail}

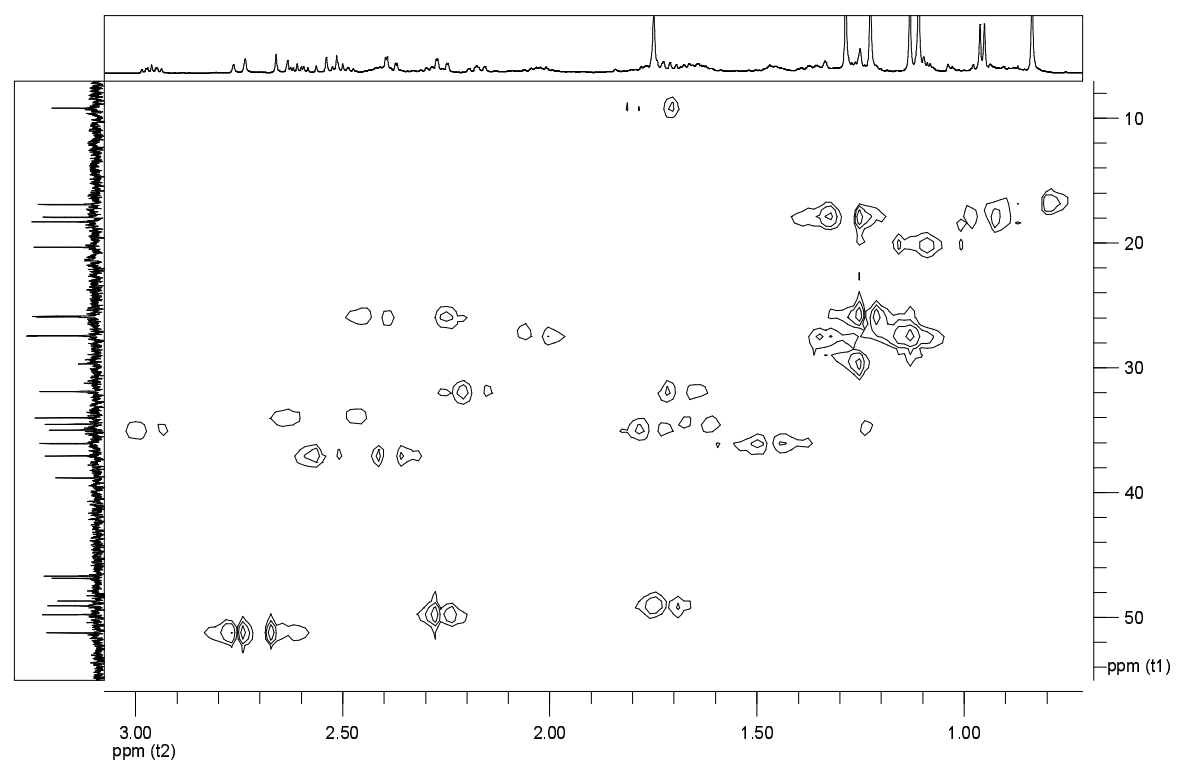

Figure 3-22 HSQC Lucialdehyde D in $\mathrm{CDCl}_{3}$ (0.7 to $3.1\left({ }^{1} \mathrm{H}\right), 7$ to $55 \mathrm{ppm}\left({ }^{13} \mathrm{C}\right)$ ) 


\subsection{7 $\mathrm{HMBC}$}

\subsubsection{HMBC overview}

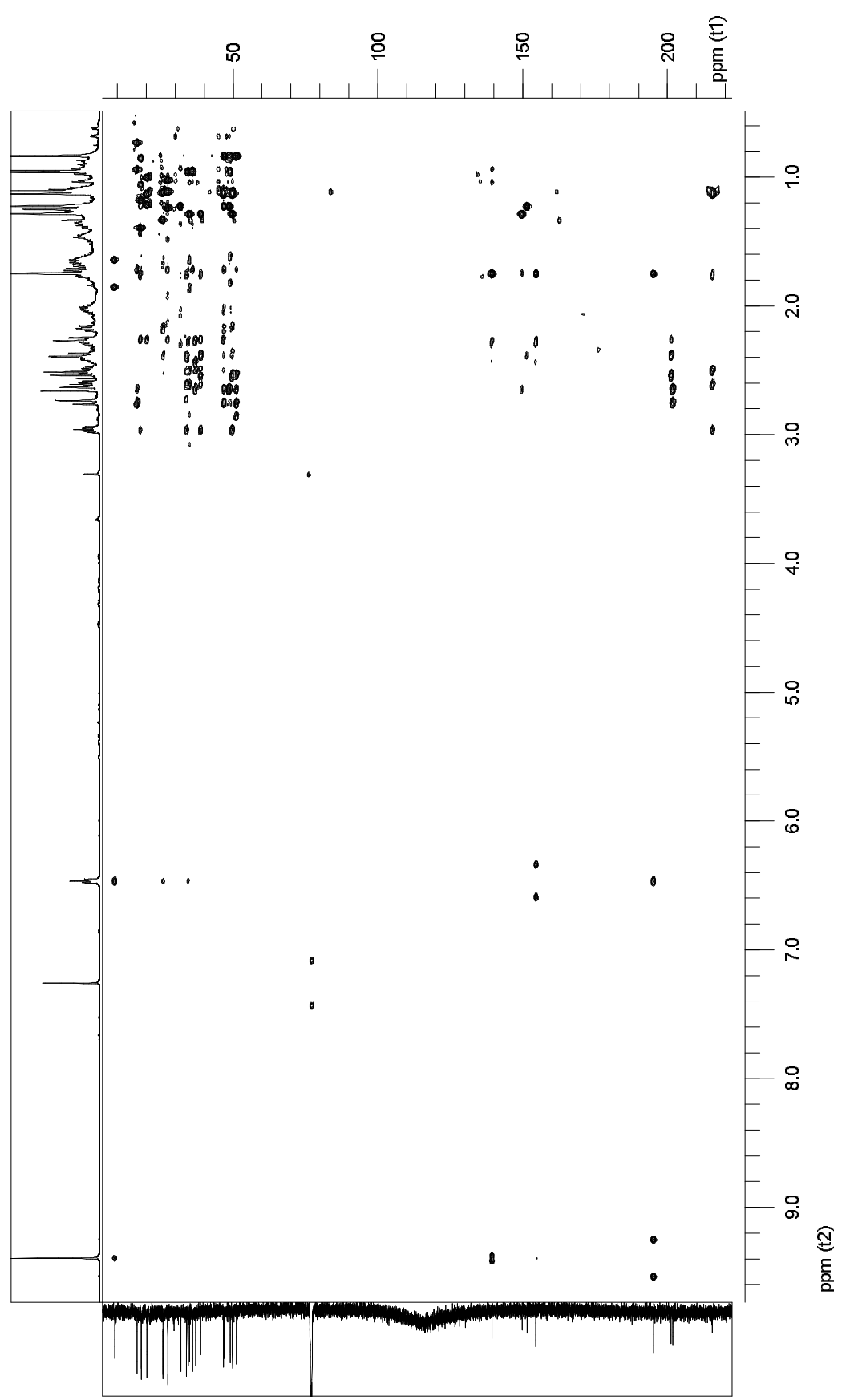

Figure 3-23 $\mathrm{HMBC}$ Lucialdehyde $\mathrm{D}$ in $\mathrm{CDCl}_{3}$ (overview) 


\subsubsection{HMBC detail}

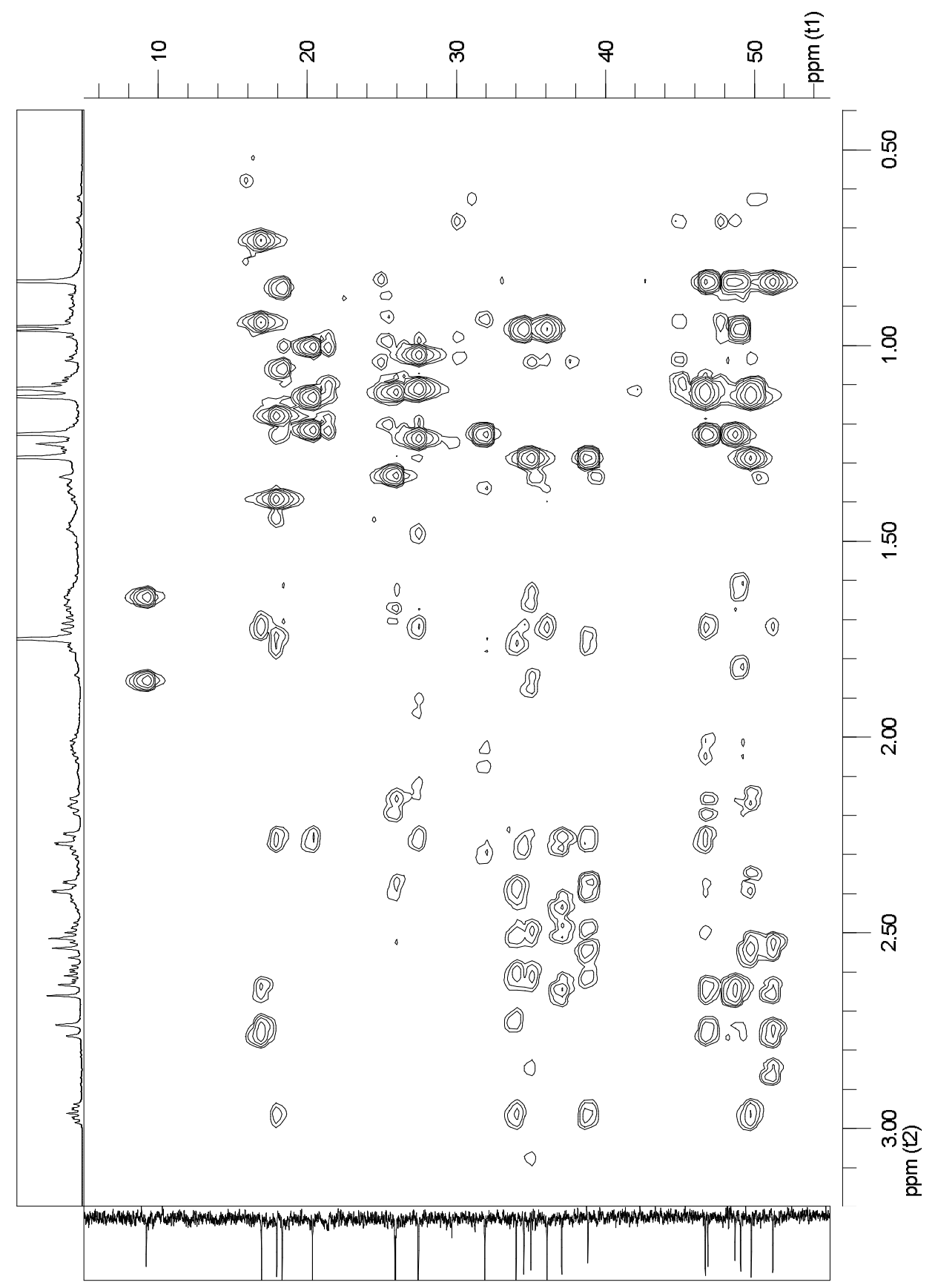

Figure 3-24 HMBC Lucialdehyde $\mathrm{D}$ in $\mathrm{CDCl}_{3}\left(0.4\right.$ to $3.2\left({ }^{1} \mathrm{H}\right), 5$ to $\left.55 \mathrm{ppm}\left({ }^{13} \mathrm{C}\right)\right)$ 


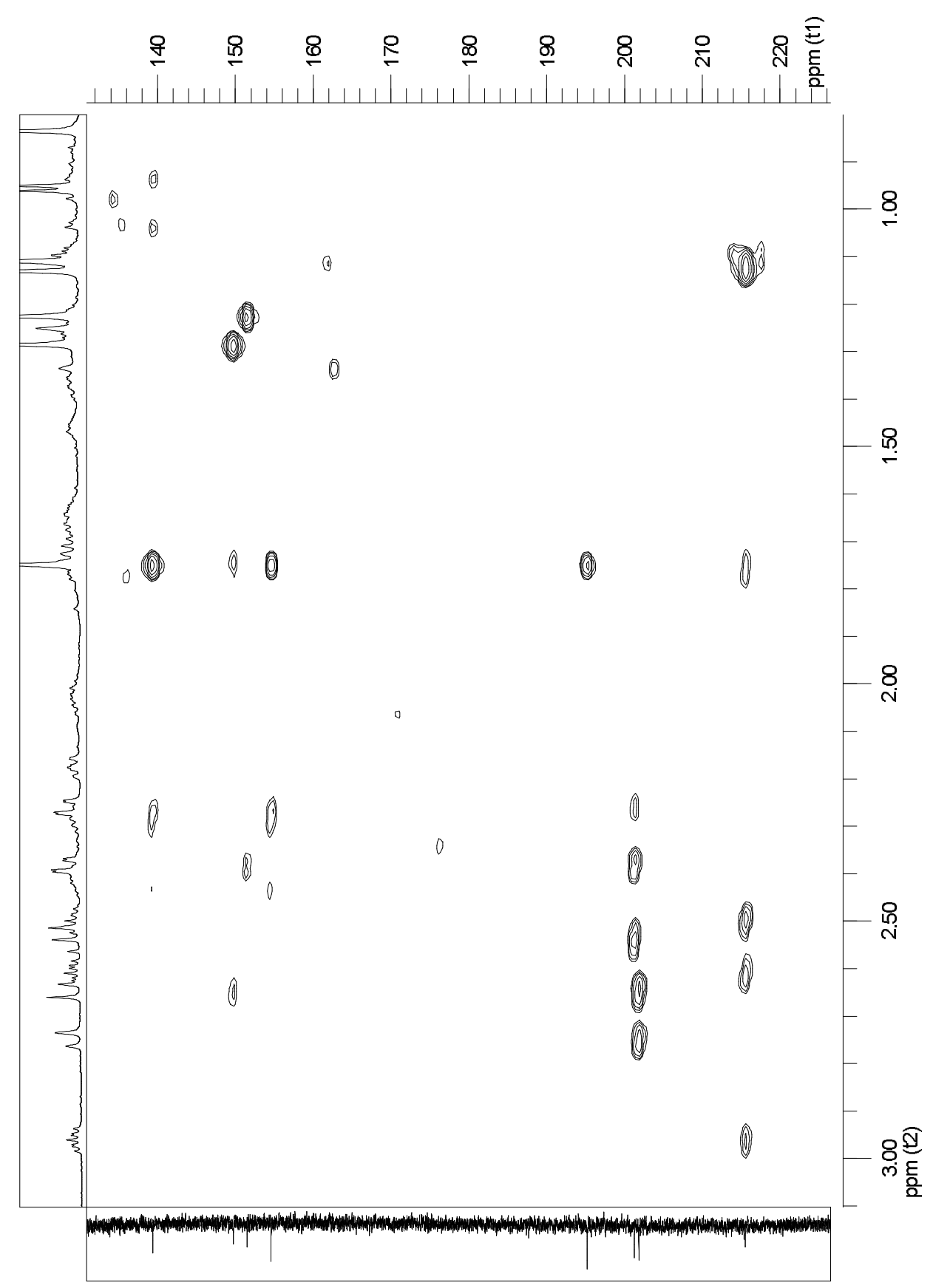

Figure 3-25 HMBC Lucialdehyde D in $\mathrm{CDCl}_{3}\left(0.8\right.$ to $3.1\left({ }^{1} \mathrm{H}\right), 131$ to $\left.226 \mathrm{ppm}\left({ }^{13} \mathrm{C}\right)\right)$ 


\subsubsection{HMBC correlations}

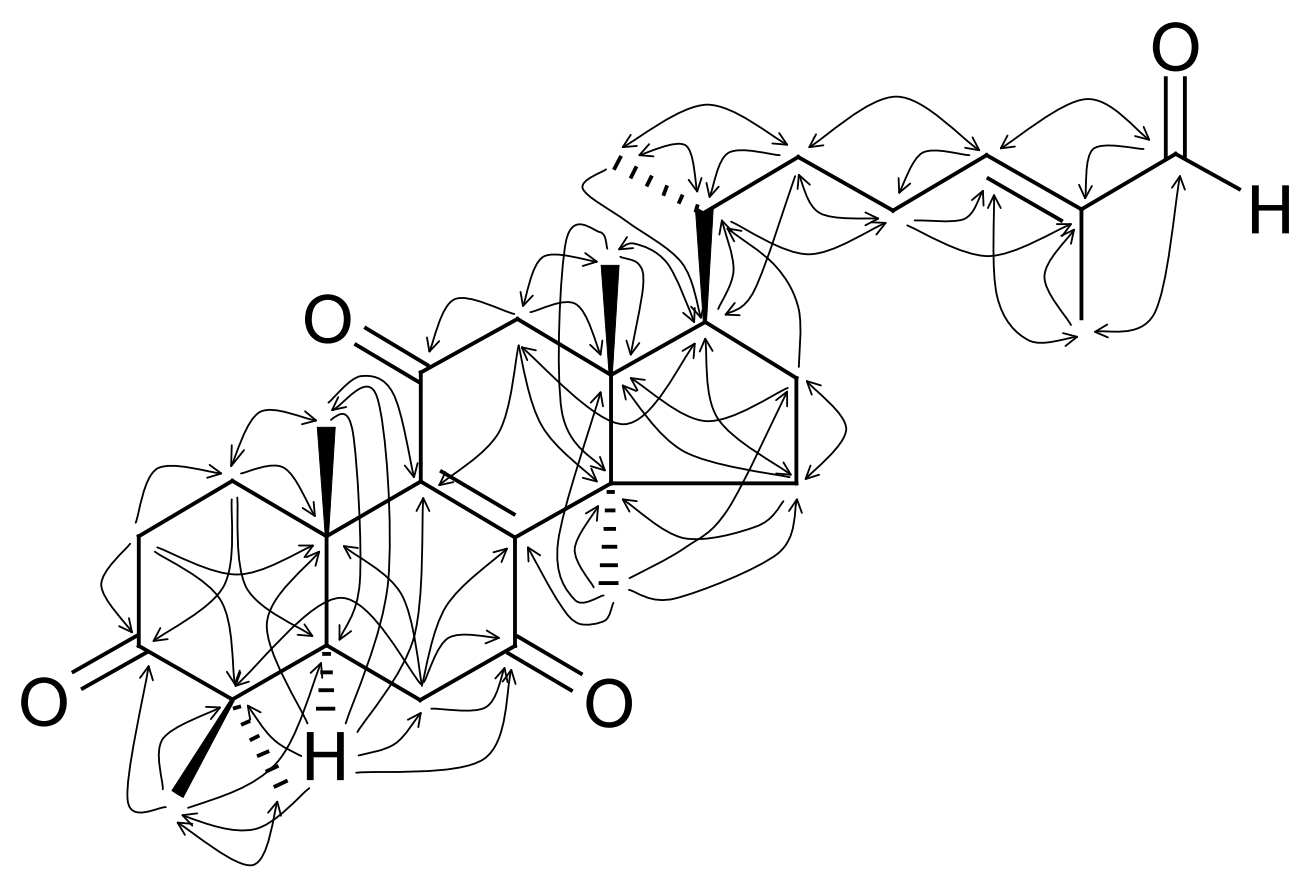

Figure 3-26 HMBC correlations Lucialdehyde D 


\subsubsection{NOESY}

\subsubsection{NOESY overview}

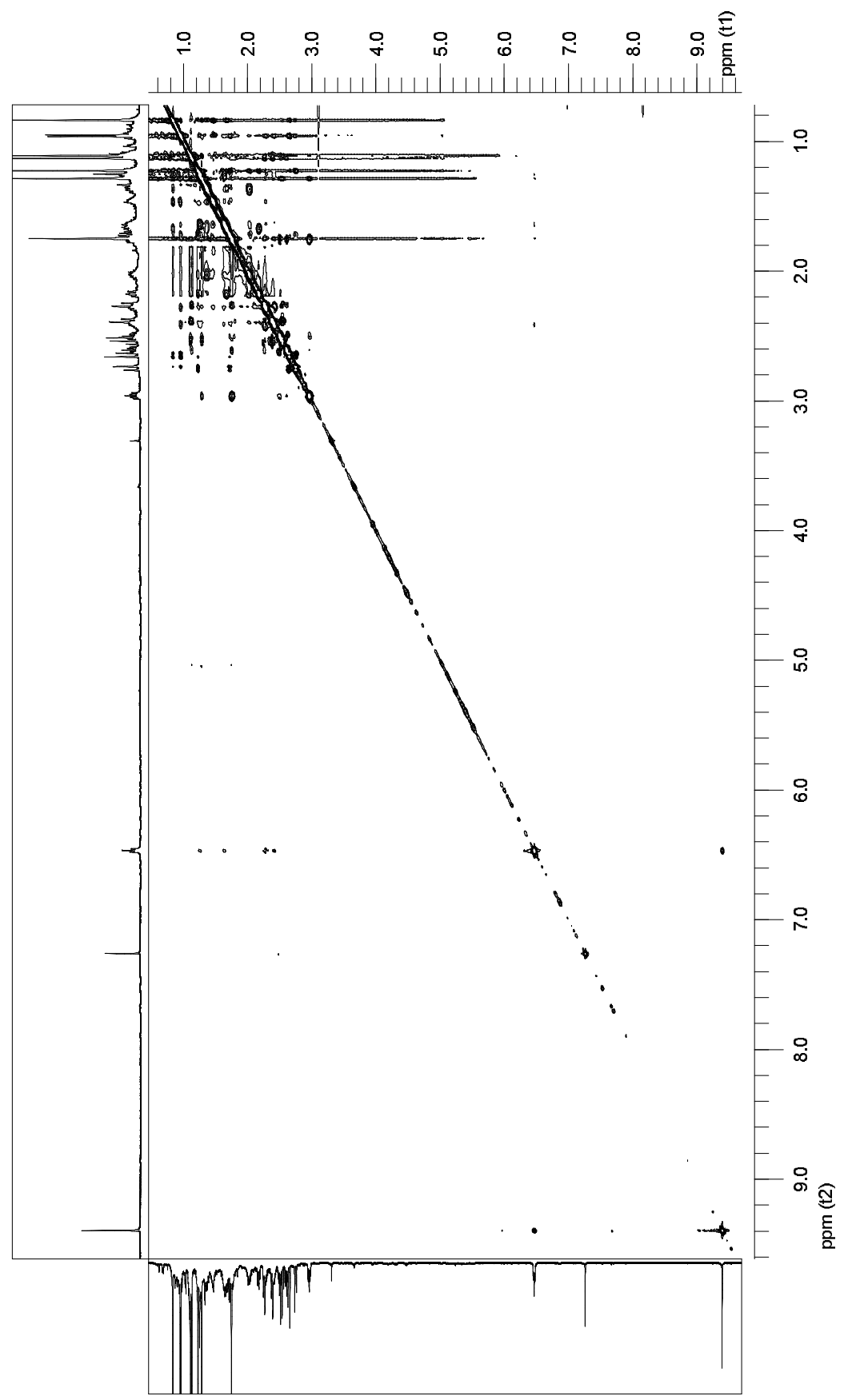

Figure 3-27 NOESY correlations Lucialdehyde $\mathrm{D}$ in $\mathrm{CDCl}_{3}$ (overview) 


\subsubsection{NOESY detail}

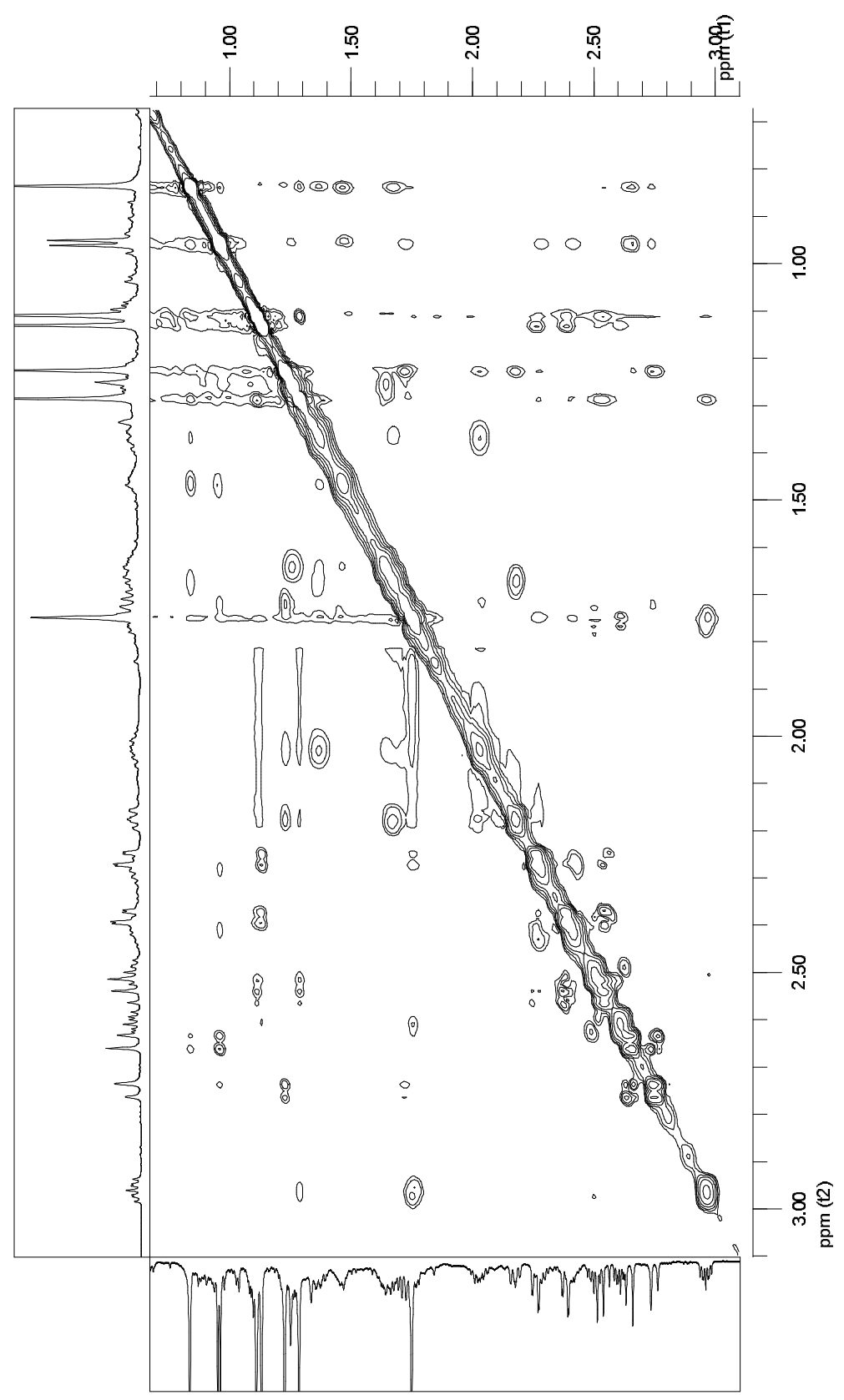

Figure 3-28 NOESY correlations Lucialdehyde D in $\mathrm{CDCl}_{3}\left(0.67\right.$ to $3.1 \mathrm{ppm}\left({ }^{1} \mathrm{H}\right)$ 


\subsection{9 similar natural compounds}

- Ganoderal B ${ }^{36}$

- Lucialdehyde $\mathrm{B}^{37}$ and this article

- Ganoderic acid $\mathrm{E}^{38}$

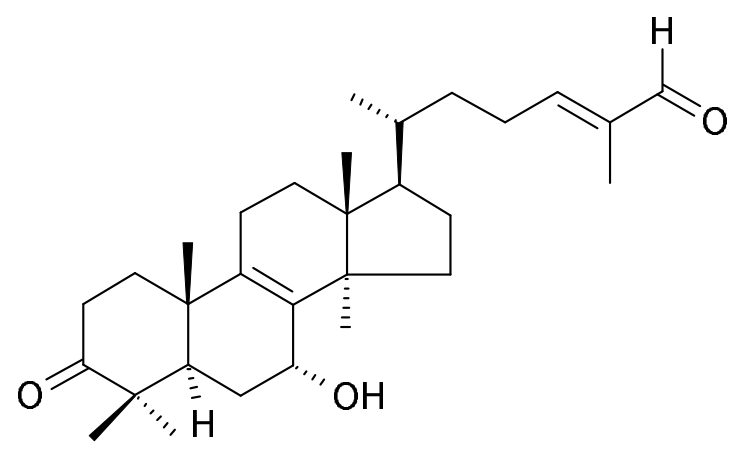

Figure 3-29 Ganoderal B

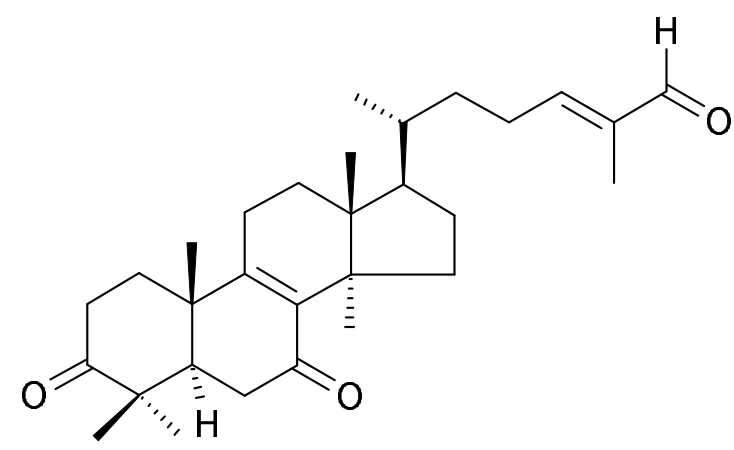

Figure 3-30 Lucialdehyde B<smiles>CC(CC(=O)C[C@@H](C)C1CC(=O)[C@]2(C)C3=C(C(=O)C[C@]12C)[C@@]1(C)CCC(=O)C(C)(C)[C@H]1CC3=O)C(=O)O</smiles>

Figure 3-31 Ganoderone C 


\subsection{Lucialdehyde B}

Isolation see Figure 2-1 on page 2. white ceraeous plates (acetone). yield $52.4 \mathrm{mg}$ (min. 0.0256 $\%$ in dried fruiting bodies of G. pfeifferi). TLC: $\mathrm{R}_{\mathrm{f}} 0.61$; fluorescence quenching at $254 \mathrm{~nm}$, no fluorescence at $366 \mathrm{~nm}$; weak purple-gray with anisaldehyde-sulphuric acid reagent.

HRFTICRMS [M+H] ${ }^{+}$: calcd for $\mathrm{C}_{30} \mathrm{H}_{45} \mathrm{O}_{3}$ : 453.3369; found: 453.3376 .

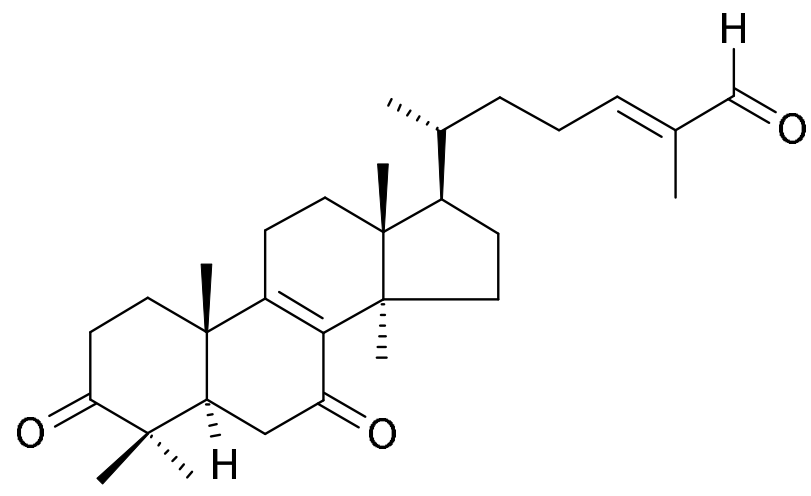

(7) Lucialdehyde B

\subsubsection{UV-vis}

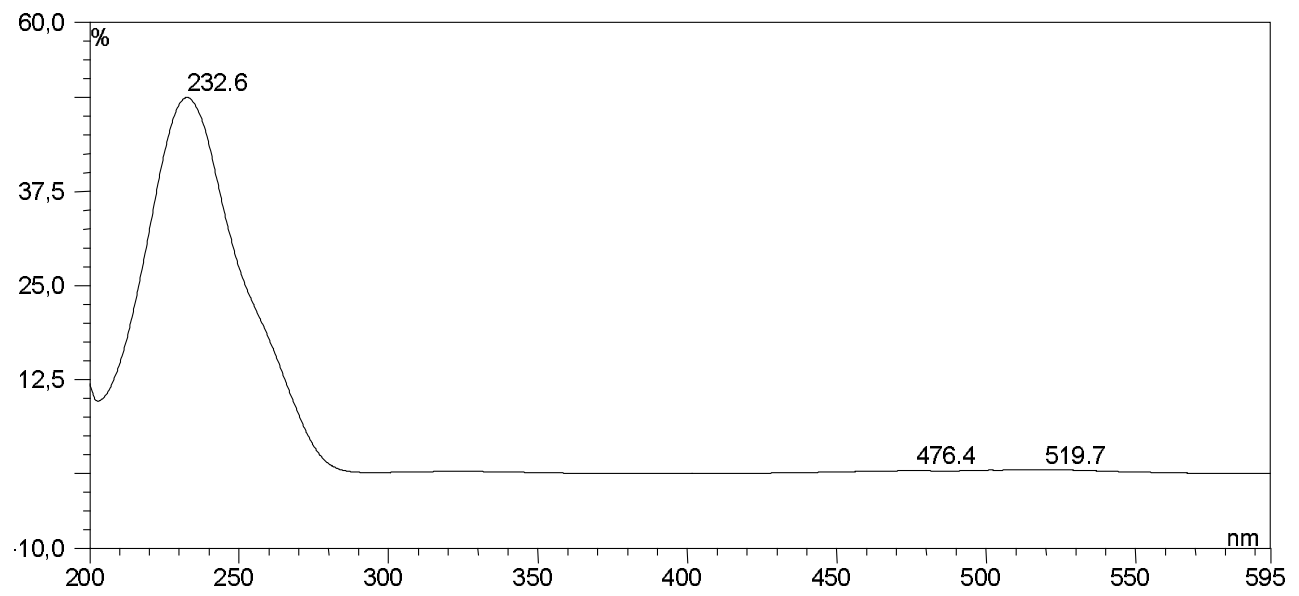

Figure 3-32 UV-vis Lucialdehyde B 


\subsubsection{EI-MS}

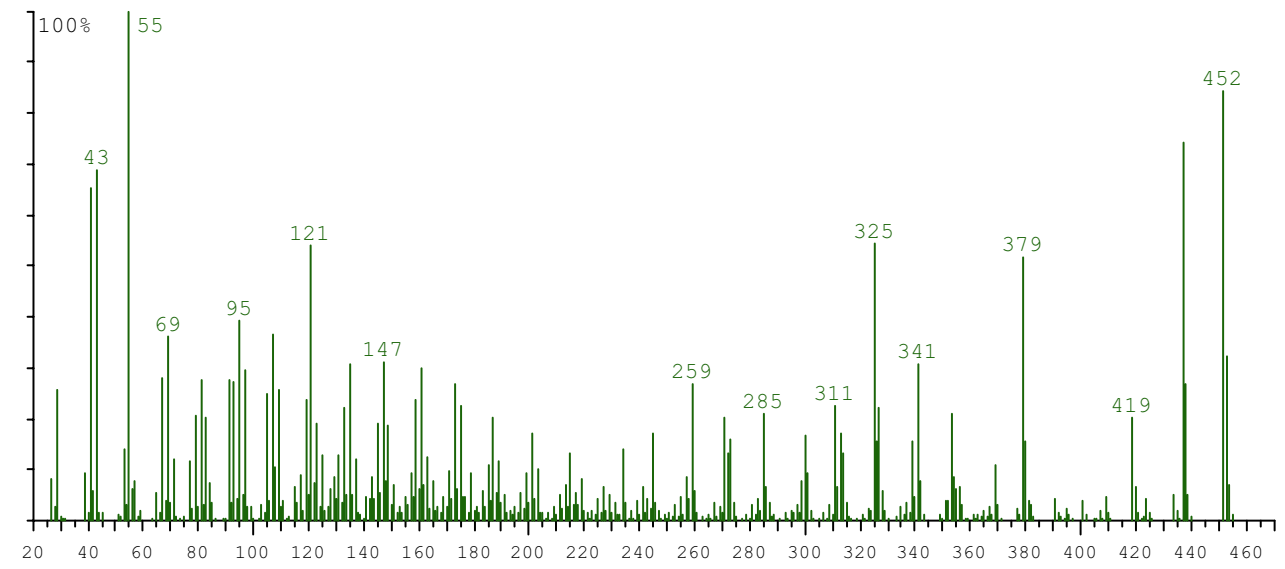

Figure 3-33 EI-MS Lucialdehyde B

\subsection{3 ${ }^{1} \mathrm{H}-\mathrm{NMR}$}

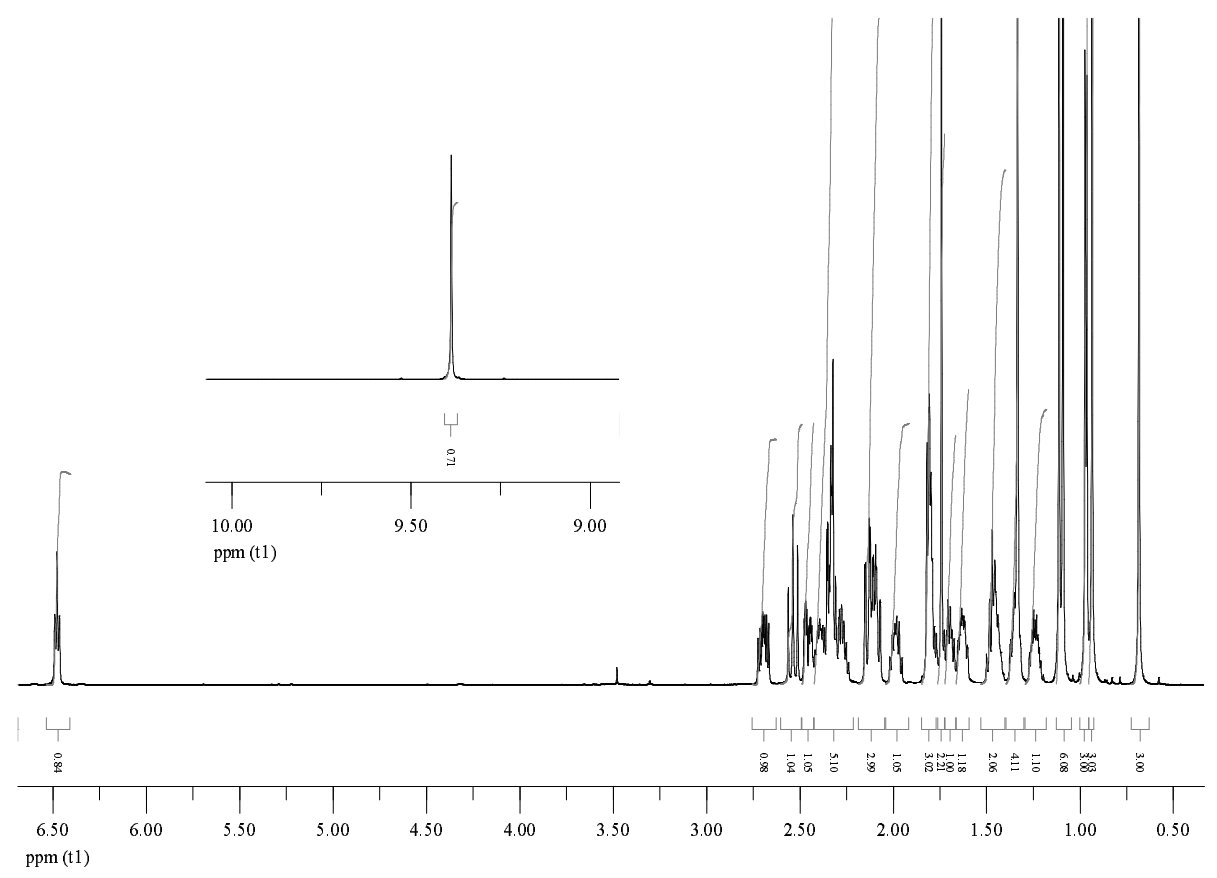

Figure 3-34 ${ }^{1} \mathrm{H}$-NMR Lucialdehyde $\mathrm{B}$ in $\mathrm{CDCl}_{3}$ 


\subsection{4 ${ }^{13} \mathrm{C}-\mathrm{NMR}$}

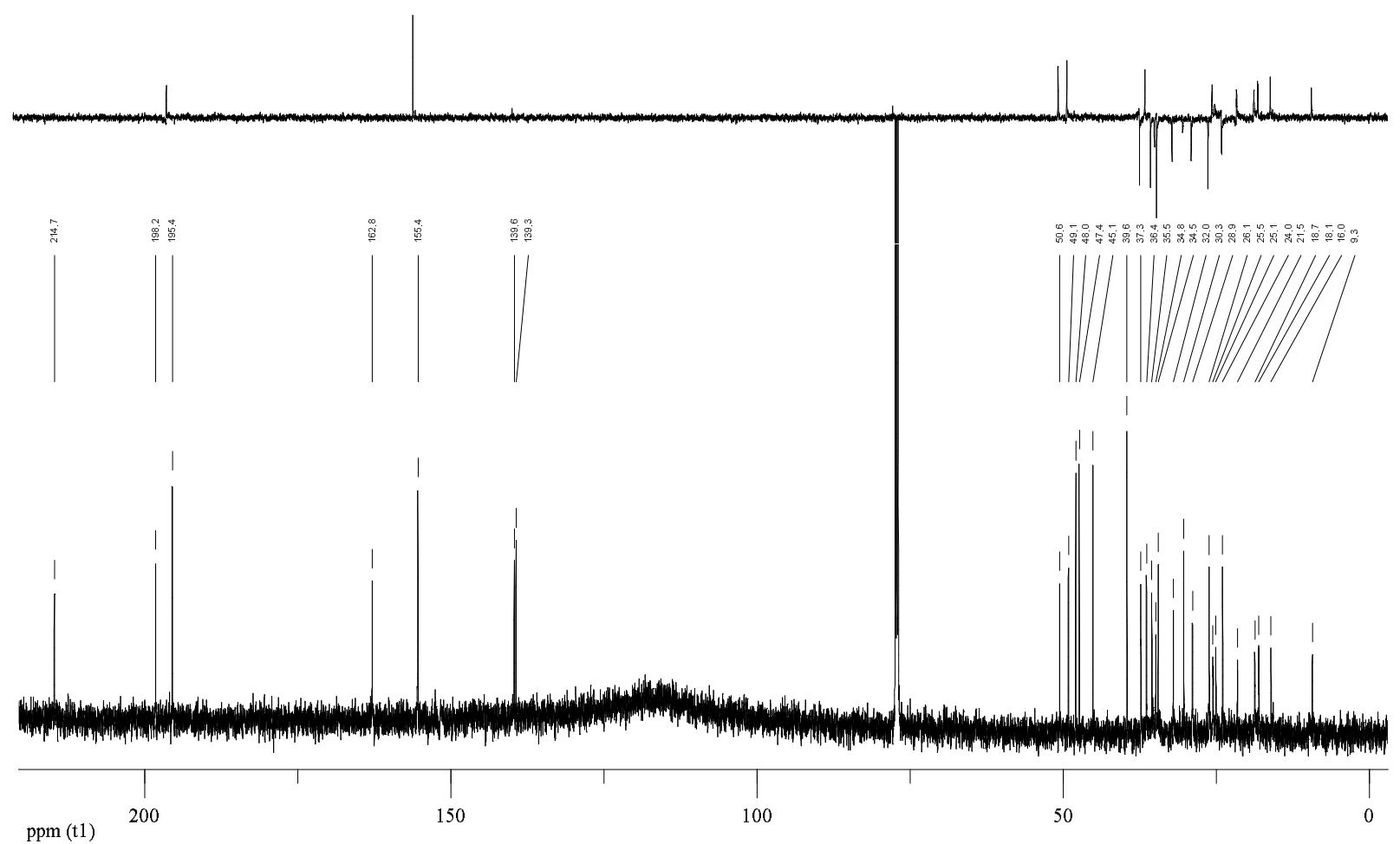

Figure 3-35 ${ }^{13} \mathrm{C}-\mathrm{NMR}$ and DEPT135 Lucialdehyde $\mathrm{B}$ in $\mathrm{CDCl}_{3}$

\subsubsection{Literature}

\begin{tabular}{ccclll} 
year & isolated from & yield $(\%)$ & structure analysis & biol. activity & in \\
\hline 2002 & G. lucidum & 0.0001 & {$[\alpha]_{\mathrm{D}}$, UV-vis, IR, EI- } & cytotoxicity againgst four tumor & 37 \\
& & & MS, ${ }^{1} \mathrm{H}-,{ }^{13}$ C-NMR & cell lines $\left(\right.$ EC $\left._{50} 4->20 \mu \mathrm{g} / \mathrm{mL}\right)$ &
\end{tabular}

Table 3-5 Literature Lucialdehyde B 


\subsection{Ganoderol A / Ganodermenonol}

Isolation see Figure 2-1 on page 2. pearly plates (acetone). yield $27.4 \mathrm{mg}$ (min. $0.0126 \%$ in dried fruiting bodies of $G$. pfeifferi). TLC: $\mathrm{R}_{\mathrm{f}} 0.61$; fluorescence quenching at $254 \mathrm{~nm}$, no fluorescence at $366 \mathrm{~nm}$; red-violet with anisaldehyde-sulphuric acid reagent.

HRFTICRMS [M $+\mathrm{H}]^{+}$: calcd for $\mathrm{C}_{30} \mathrm{H}_{47} \mathrm{O}_{2}$ : 439.3576; found: 439.3580 .

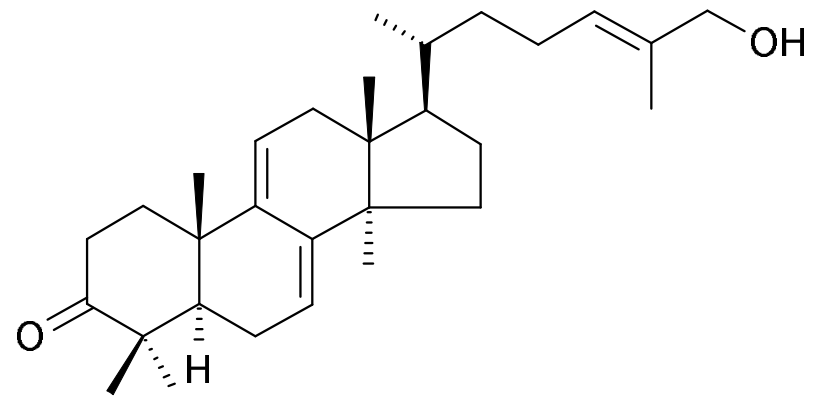

(5) Ganoderol A

\subsubsection{UV-vis}

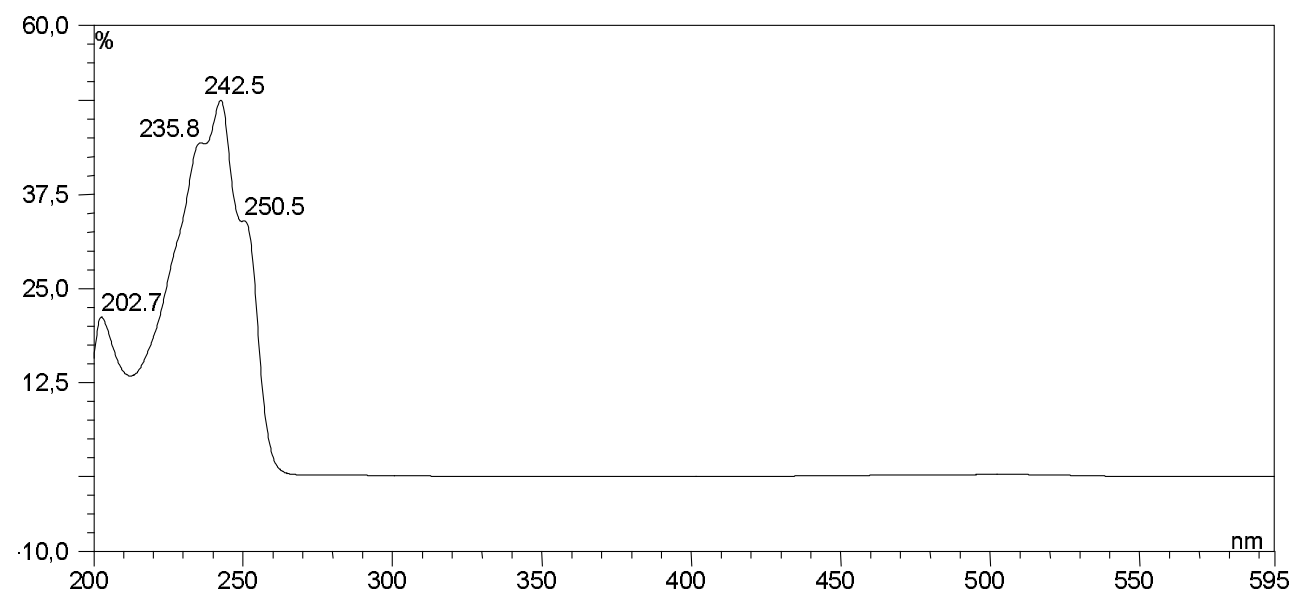

Figure 3-36 UV-vis Ganoderol A 


\subsubsection{EI-MS}

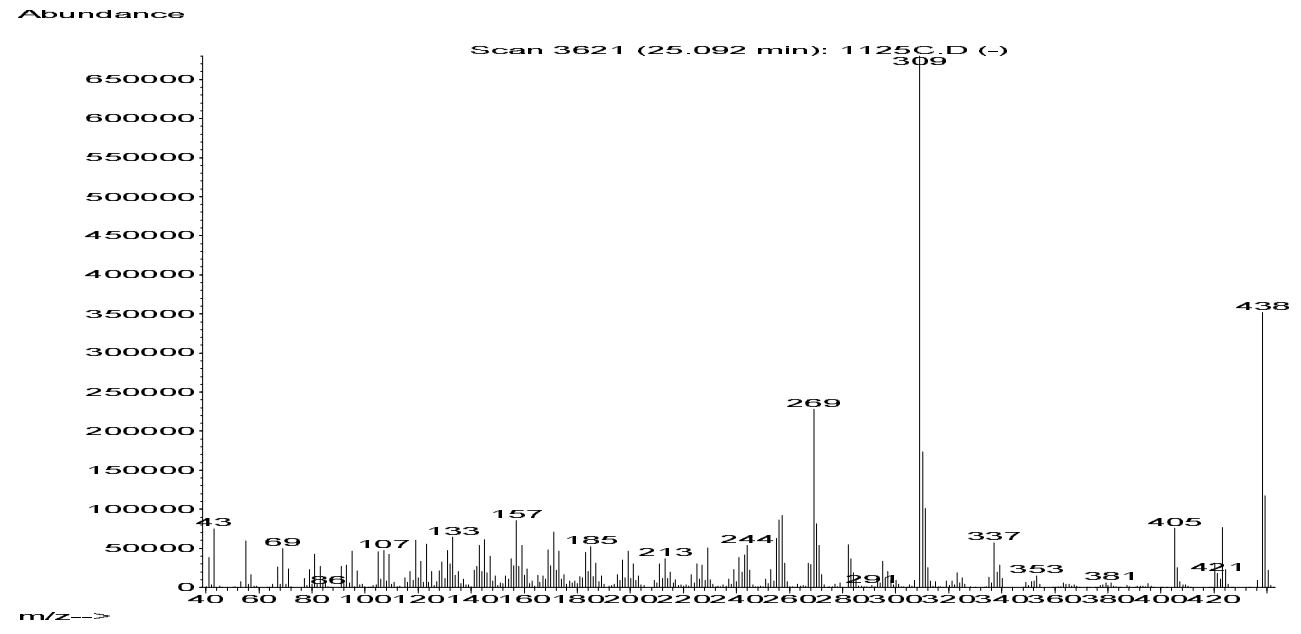

Figure 3-37 EI-MS Ganoderol A

\subsection{3 ${ }^{1} \mathrm{H}-\mathrm{NMR}$}

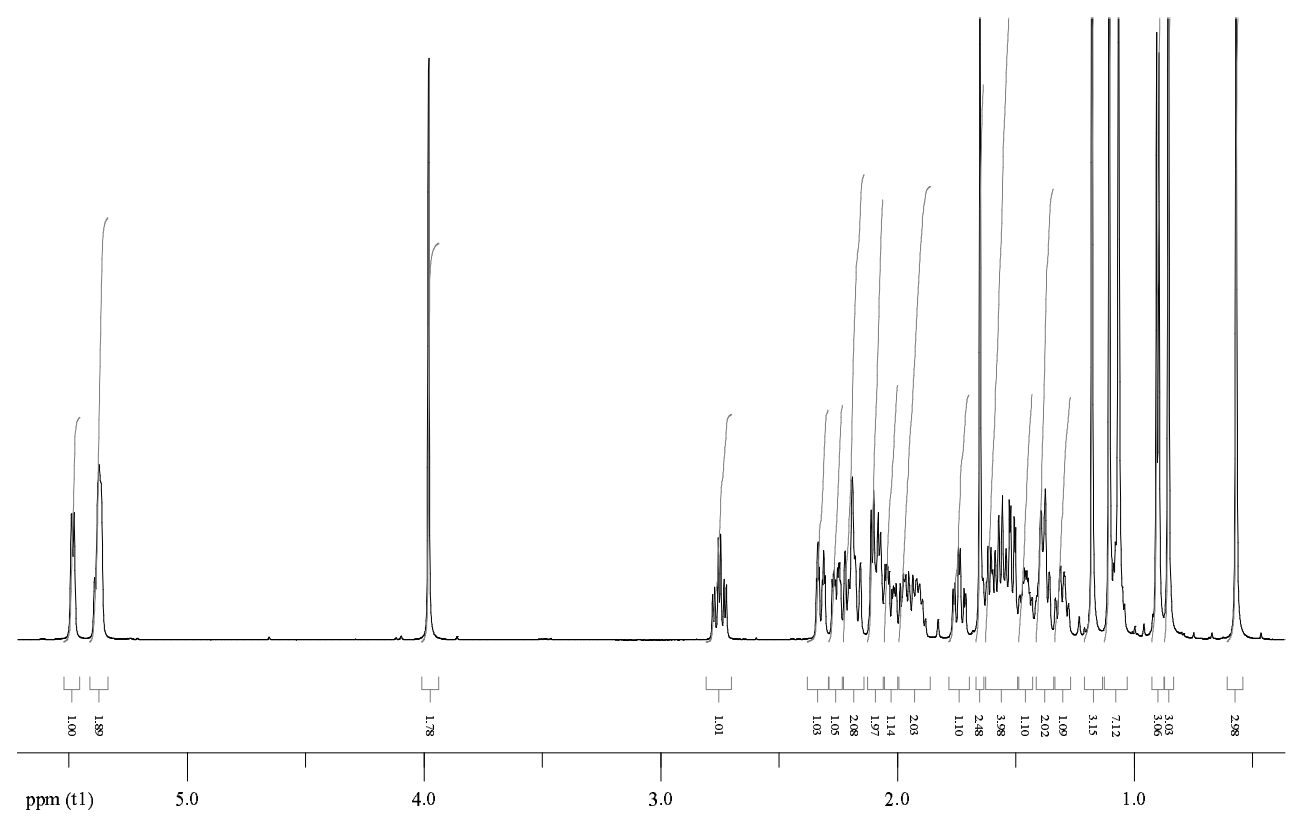

Figure 3-38 ${ }^{1} \mathrm{H}-\mathrm{NMR}$ Ganoderol $\mathrm{A}$ in $\mathrm{CDCl}_{3}$ 


\subsection{4 ${ }^{13} \mathrm{C}-\mathrm{NMR}$}

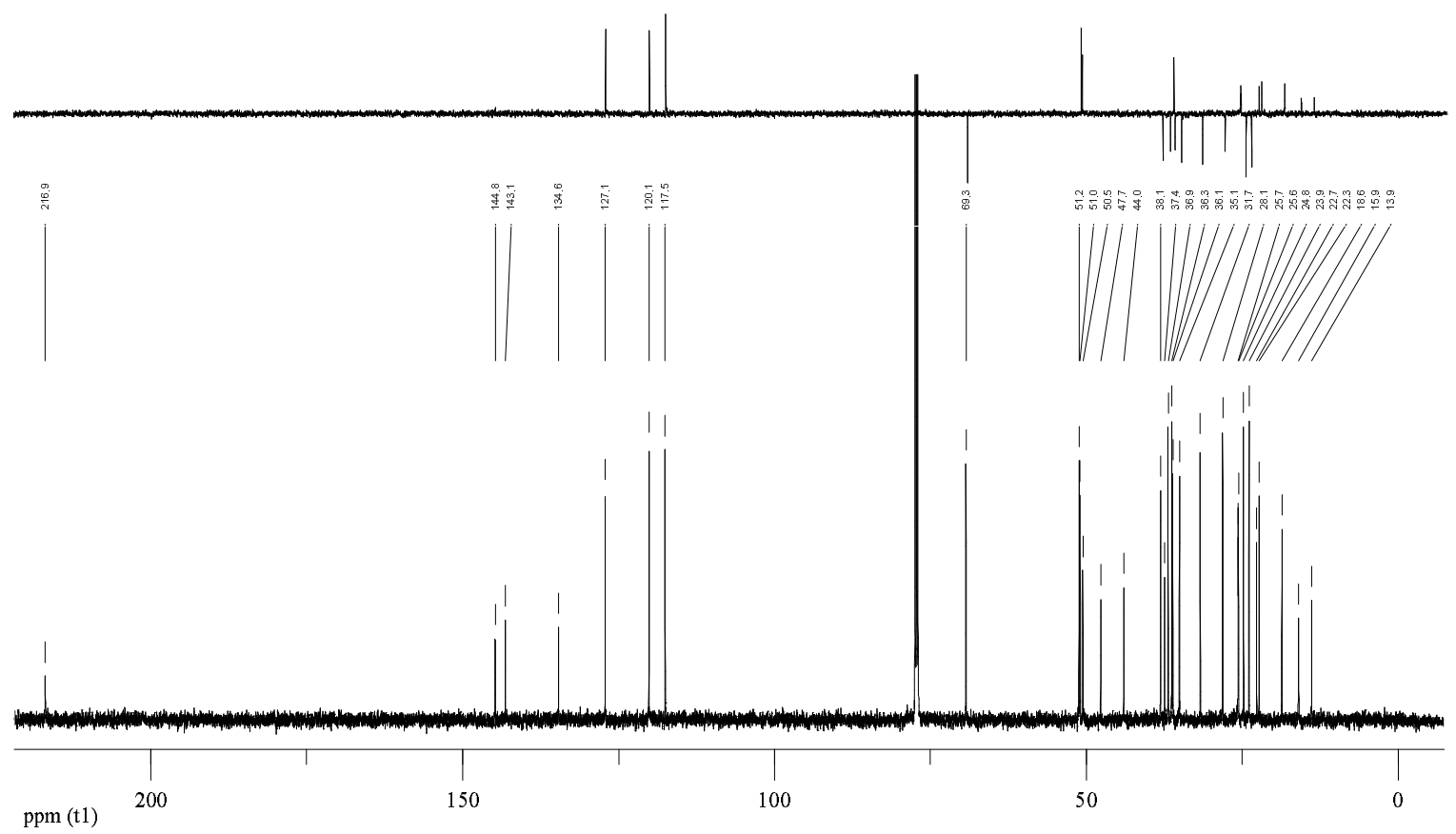

Figure 3-39 ${ }^{13} \mathrm{C}-\mathrm{NMR}$ and DEPT135 Ganoderol $\mathrm{A}$ in $\mathrm{CDCl}_{3}$

\subsubsection{Literature}

\begin{tabular}{|c|c|c|c|c|c|}
\hline year & isolated from & yield $(\%)$ & structure analysis & biol. activity & in \\
\hline 1986 & G. lucidum & $\mathrm{n} / \mathrm{a}$ & $\begin{array}{l}\mathrm{mp},[\alpha]_{\mathrm{D}}, \mathrm{UV}-\mathrm{vis}, \mathrm{R}_{\mathrm{f}} \\
\text { EI-MS, }{ }^{1} \mathrm{H}-\mathrm{NMR}\end{array}$ & $\begin{array}{l}\text { inhibition of angiotensin convert- } \\
\text { ing enzyme }\left(\mathrm{IC}_{50}>10^{-4} \mathrm{M}\right)\end{array}$ & 8 \\
\hline 1986 & G. lucidum & 0.0004 & $\begin{array}{l}\mathrm{mp},[\alpha]_{\mathrm{D}}, \mathrm{IR}, \mathrm{UV}-\mathrm{vis}, \\
\text { EI-MS, }{ }^{1} \mathrm{H}-,{ }^{13} \mathrm{C}-\mathrm{NMR}\end{array}$ & $\mathrm{n} / \mathrm{a}$ & 39 \\
\hline 1987 & G. lucidum & $0.09-0.17$ & $\begin{array}{l}\mathrm{mp}, \text { EI-MS, IR, }{ }^{1} \mathrm{H}-, \\
{ }^{13} \mathrm{C}-\mathrm{NMR}\end{array}$ & $\mathrm{n} / \mathrm{a}$ & 9 \\
\hline 1988 & $\mathrm{n} / \mathrm{a}$ & $\mathrm{n} / \mathrm{a}$ & ${ }^{1} \mathrm{H}-,{ }^{13} \mathrm{C}-\mathrm{NMR}$ & $\mathrm{n} / \mathrm{a}$ & 40 \\
\hline 2002 & G. concinna & 0.0176 & $\mathrm{n} / \mathrm{a}$ & $\mathrm{n} / \mathrm{a}$ & 7 \\
\hline 2002 & G. lucidum & 0.0009 & $\mathrm{n} / \mathrm{a}$ & $\begin{array}{l}\text { cytotoxicity againgst four tumor } \\
\text { cell lines }\left(\mathrm{EC}_{50} 3->20 \mu \mathrm{g} / \mathrm{mL}\right)\end{array}$ & 37 \\
\hline
\end{tabular}

Table 3-6 Literature Ganoderol A 


\section{$3.85 \alpha, 8 \alpha$-Epidioxyergosta-6,22-diene-3 $\beta$-ol}

Isolation see Figure 2-1 on page 2. yield $74.8 \mathrm{mg}$ (min. $0.0346 \%$ in dried fruiting bodies of $G$. pfeifferi). TLC: $\mathrm{R}_{\mathrm{f}} 0.46$; fluorescence quenching at $254 \mathrm{~nm}$, weak yellow fluorescence at $366 \mathrm{~nm}$; black with anisaldehyde-sulphuric acid reagent.

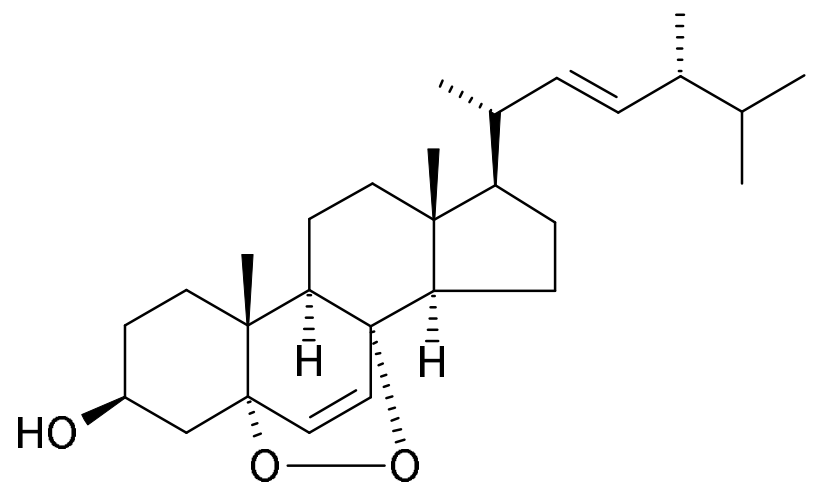

$5 \alpha, 8 \alpha$-Epidioxyergosta-6,22-dien-3 $\beta$-ol

\subsubsection{El-MS}

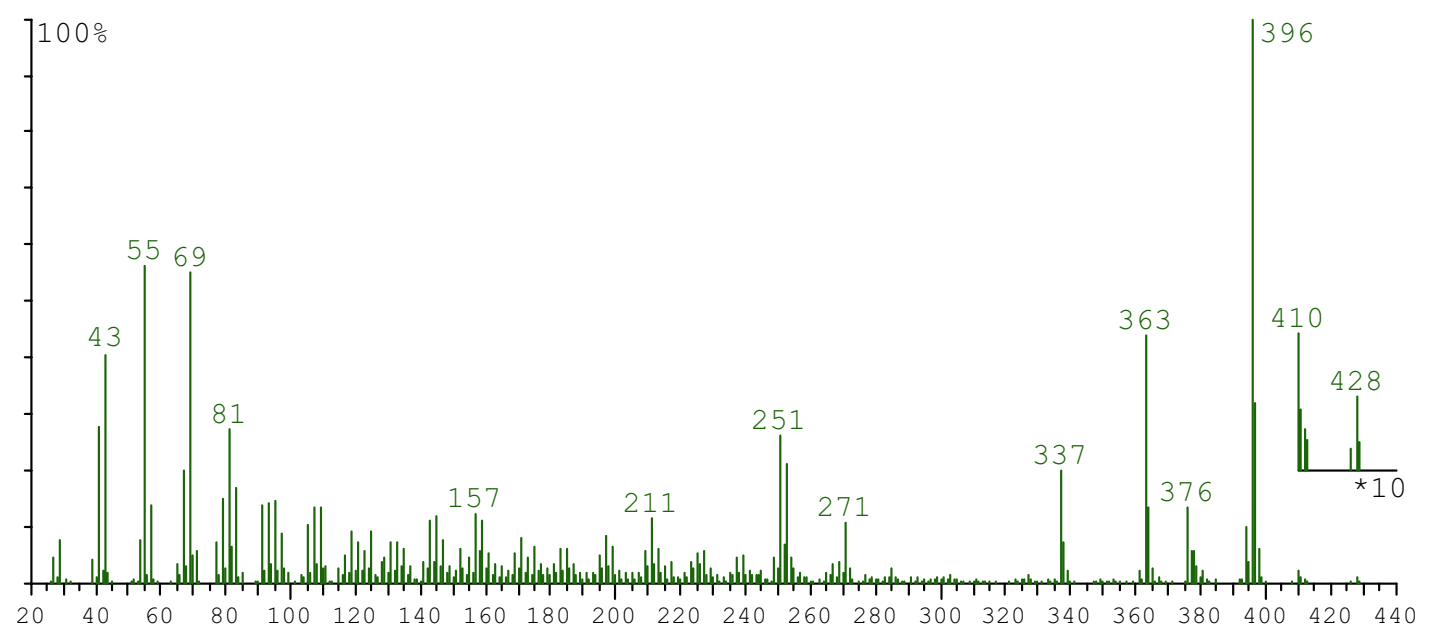

Figure 3-40 EI-MS $5 \alpha, 8 \alpha$-Epidioxyergosta-6,22-dien-3 $\beta$-ol 


\subsection{2 ${ }^{1} \mathrm{H}-\mathrm{NMR}$}

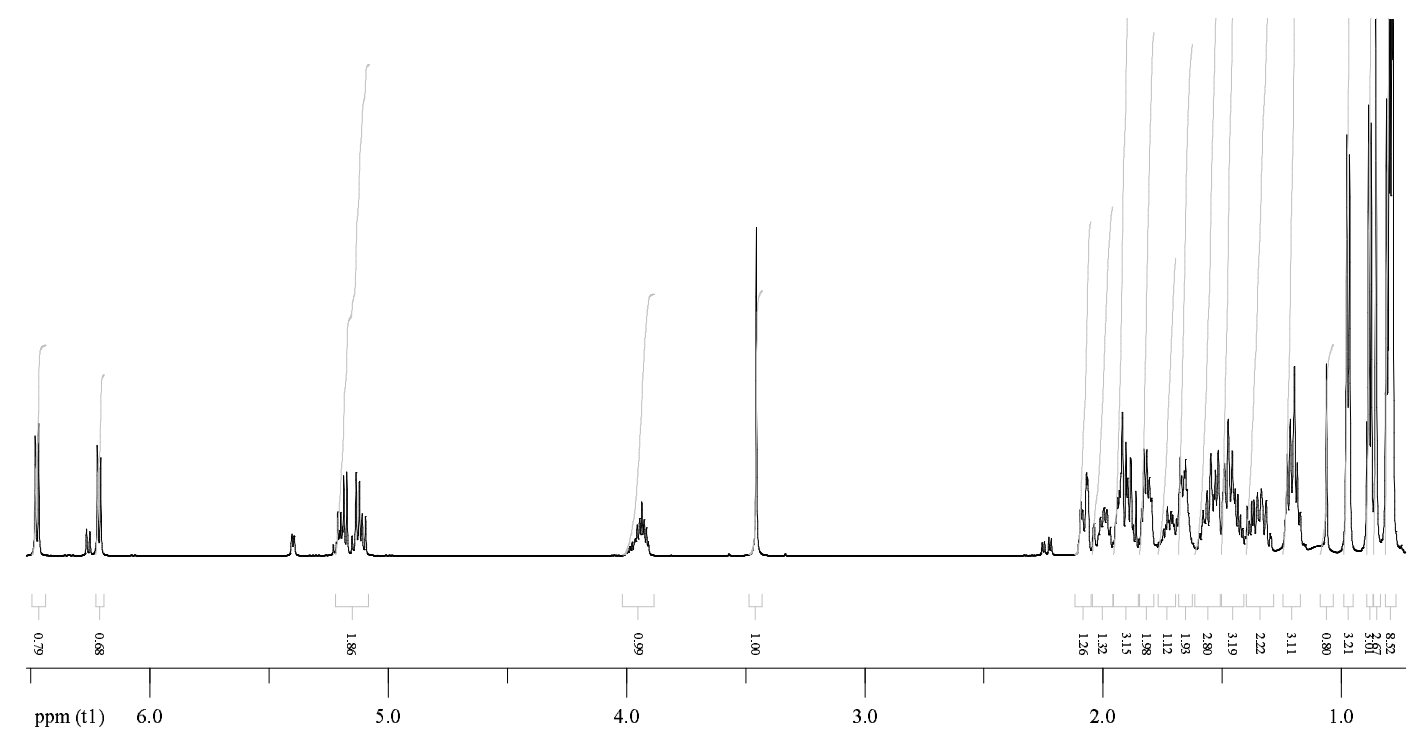

Figure 3-41 ${ }^{1} \mathrm{H}$-NMR $5 \alpha, 8 \alpha$-Epidioxyergosta-6,22-dien- $3 \beta$-ol in $\mathrm{CDCl}_{3}$

\subsection{3 ${ }^{13} \mathrm{C}-\mathrm{NMR}$}

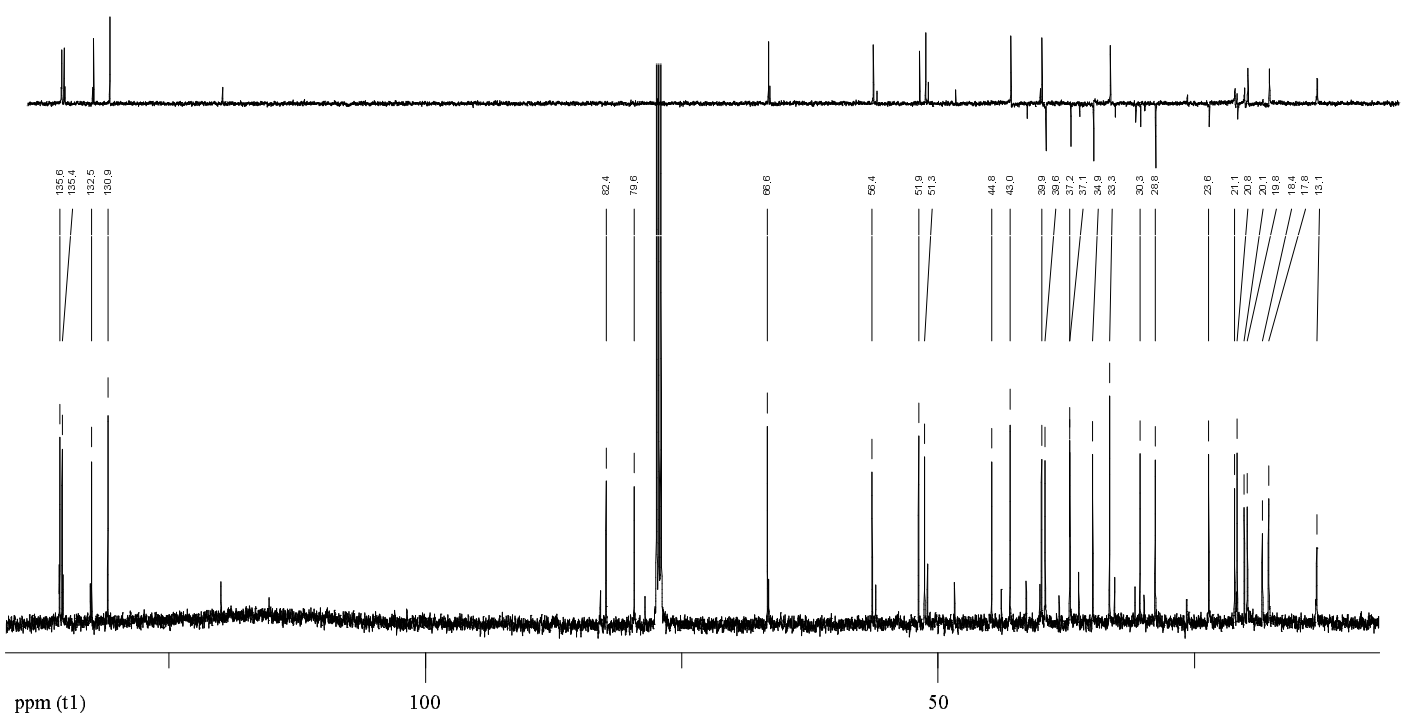

Figure 3-42 ${ }^{13} \mathrm{C}$-NMR and DEPT135 $5 \alpha, 8 \alpha$-Epidioxyergosta-6,22-dien-3 $\beta$-ol in $\mathrm{CDCl}_{3}$ 


\subsubsection{Literature}

$5 \alpha, 8 \alpha$-Epidioxyergosta-6,22-dien-3 $\beta$-ol has been found in many fungi, as well as in plants, ${ }^{41-46}$ sponges $^{47,48}$ and other marine organisms. ${ }^{48}$ Therefore, only a small number of interesting references is given here.

\begin{tabular}{|c|c|c|c|c|c|}
\hline year & isolated from & yield $(\%)$ & structure analysis & biol. activity & in \\
\hline 1928 & synthesis & yield $70 \%$ & $\begin{array}{l}\text { general proper- } \\
\text { ties and colour } \\
\text { reactions, } \mathrm{mp},[\alpha]_{\mathrm{D}}\end{array}$ & no antirachitic properties & 49 \\
\hline 1947 & Aspergillus fumigatus & $\mathrm{n} / \mathrm{a}$ & $\operatorname{mp},[\alpha]_{\mathrm{D}}$ & $\mathrm{n} / \mathrm{a}$ & 50 \\
\hline 1985 & Polyporus umbellatus & 0.0106 & $\begin{array}{l}\mathrm{mp}, \quad[\alpha]_{\mathrm{D}}, \quad \text { IR, EI- } \\
\mathrm{MS},{ }^{1} \mathrm{H}-\mathrm{NMR}\end{array}$ & $\begin{array}{l}\text { enhancing effect on collagen } \\
\text { induced rabbit platelet ag- } \\
\text { gregation }\end{array}$ & 4 \\
\hline 1989 & synthesis & $\mathrm{n} / \mathrm{a}$ & $\mathrm{n} / \mathrm{a}$ & antiviral activity & 51 \\
\hline 1989 & Inonotus radiatus & $\mathrm{n} / \mathrm{a}$ & $\mathrm{n} / \mathrm{a}$ & $\begin{array}{l}\text { cytotoxic against two tumor } \\
\text { cell lines }\left(\mathrm{EC}_{50} 4-10 \mu \mathrm{g} / \mathrm{mL}\right)\end{array}$ & 24 \\
\hline 1990 & Typha latifolia & $\mathrm{n} / \mathrm{a}$ & $\begin{array}{l}\text { mp, } \quad[\alpha]_{\mathrm{D}}, \quad \text { EI-MS, } \\
{ }^{1} \mathrm{H}-,{ }^{13} \mathrm{C}-\mathrm{NMR}\end{array}$ & $\mathrm{n} / \mathrm{a}$ & 41 \\
\hline 1991 & Meripilus giganteus & $\mathrm{n} / \mathrm{a}$ & EI-MS, IR & $\begin{array}{l}\text { inhibition of PHA stimulated } \\
\text { proliferation of human lym- } \\
\text { phocytes }\end{array}$ & 52 \\
\hline 1991 & synthesis & $\mathrm{n} / \mathrm{a}$ & $\mathrm{n} / \mathrm{a}$ & $\begin{array}{l}\text { inhibitory against human } \\
\text { hepatoma } \mathrm{PLC} / \mathrm{PRF} / 5 \text { cells } \\
\left(\mathrm{ED}_{50} 11 \mu \mathrm{g} / \mathrm{mL}\right) \text { and } \mathrm{KB} \\
\text { cells }\left(\mathrm{ED}_{50} 10 \mu \mathrm{g} / \mathrm{mL}\right)\end{array}$ & 53 \\
\hline 1994 & $\begin{array}{l}\text { Pisolithus tinctorius } \\
\text { Microporus flabellifor- } \\
\text { mis }\end{array}$ & $\begin{array}{l}0.0003 \\
0.0078\end{array}$ & $\mathrm{mp},[\alpha]_{\mathrm{D}}$ & $\begin{array}{l}\text { suppression of the prolifera- } \\
\text { tion of mouse spleen lympho- } \\
\text { cytes stimulated with Con A }\end{array}$ & 54 \\
\hline & Lenzites betulina & 0.0061 & & $\begin{array}{l}\left(\mathrm{IC}_{50} 2.6 \mu \mathrm{g} / \mathrm{mL}\right) \text { and LPS } \\
\left(\mathrm{IC}_{50} 1.5 \mu \mathrm{g} / \mathrm{mL}\right)\end{array}$ & \\
\hline
\end{tabular}




\begin{tabular}{|c|c|c|c|c|c|}
\hline year & isolated from & yield $(\%)$ & structure analysis & biol. activity & in \\
\hline 1997 & Melilotus messanensis & $>0.0003$ & $\mathrm{n} / \mathrm{a}$ & $\begin{array}{l}\text { very weak influence on } \\
\text { growth of Lactuca sativa, } \\
\text { Lycopersicon esculentum } \\
\text { and Allium cepa, stimulation } \\
\text { of germination of Hordeum } \\
\text { vulgare }\end{array}$ & 45 \\
\hline 1998 & Zantedeschia aethiopca & $\mathrm{n} / \mathrm{a}$ & $\mathrm{n} / \mathrm{a}$ & no antialgal activity & 42 \\
\hline 1998 & G. lucidum & 0.0121 & $\mathrm{n} / \mathrm{a}$ & $\begin{array}{l}\text { no inhibition of HIV-1- } \\
\text { induced cytopathogenicity, } \\
\text { no inhibition of HIV protease }\end{array}$ & 55 \\
\hline 1998 & G. lucidum & $\mathrm{n} / \mathrm{a}$ & ${ }^{1} \mathrm{H}-,{ }^{13} \mathrm{C}-\mathrm{NMR}$ & $\begin{array}{l}\text { Potentiation of the DNA } \\
\text { polymerase } \beta \text { inhibiting ac- } \\
\text { tivity of linoleic acid, no ef- } \\
\text { fect on linoleic acid inhibi- } \\
\text { tion of DNA polymerase } \alpha\end{array}$ & 56 \\
\hline 1999 & G. applanatum & $\mathrm{n} / \mathrm{a}$ & $\mathrm{n} / \mathrm{a}$ & $\begin{array}{l}\text { weak antibacterial activity } \\
(\mathrm{MIC}(\mathrm{mg} / \mathrm{mL}) \text { i.e. } 0.5(B . \\
\text { cereus }), 1.0(E \text {. coli }), 1.0(S . \\
\text { aureus })\end{array}$ & 27 \\
\hline 1999 & Armillariella mella & 0.0133 & $\begin{array}{l}m p, R_{\mathrm{f}}, \text { EI-MS, IR, } \\
{ }^{1} \mathrm{H}-,{ }^{13} \mathrm{C}-\mathrm{NMR}, \mathrm{X}- \\
\text { ray crystallography }\end{array}$ & antioxidant activity & 57 \\
\hline 1999 & synthesis & $\mathrm{n} / \mathrm{a}$ & $\begin{array}{l}\text { IR, EI-MS, }{ }^{1} \mathrm{H}- \\
{ }^{13} \mathrm{C}-\mathrm{NMR}\end{array}$ & $\begin{array}{l}\text { cytotoxic against five tu- } \\
\text { mor cell lines }\left(\mathrm{EC}_{50} 40-70\right. \\
\mu \mathrm{g} / \mathrm{mL}) ; \text { 3-glucopyranoside } \\
\text { of }(\mathbf{6}) \text { showed higher potency } \\
\text { than the aglycon }\end{array}$ & 58 \\
\hline 1999 & Ajuga remota & 0.0031 & $\begin{array}{l}\text { single crystal X-ray } \\
\text { diffraction }\end{array}$ & $\begin{array}{l}\text { activity in a radiorespiro- } \\
\text { metric assay against My- } \\
\text { cobacterium tuberculosis } \\
(\mathrm{MIC} 1 \mu \mathrm{g} / \mathrm{mL})\end{array}$ & 46 \\
\hline 2000 & Lepiota americana & $>0.0003$ & $\mathrm{n} / \mathrm{a}$ & $\begin{array}{l}\text { inhibition of sulfatase } \\
\left(\mathrm{IC}_{50} 0.9 \mu \mathrm{M}\right) \text {, no inhibition } \\
\text { of aromatase }\end{array}$ & 59 \\
\hline
\end{tabular}




\begin{tabular}{|c|c|c|c|c|c|}
\hline year & isolated from & yield $(\%)$ & structure analysis & biol. activity & in \\
\hline 2001 & Paecilomyces tenuipes & 0.2333 & $\begin{array}{l}\mathrm{mp}, \quad[\alpha]_{\mathrm{D}}, \quad \text { EI-MS, } \\
{ }^{1} \mathrm{H}-\mathrm{NMR}\end{array}$ & $\begin{array}{l}\text { cytotoxic against four tumor } \\
\text { cell lines }\left(\mathrm{EC}_{50} 8-70 \mu \mathrm{g} / \mathrm{mL}\right)\end{array}$ & 60 \\
\hline 2002 & Paecilomyces sp. J300 & 0.0018 & $\begin{array}{l}\mathrm{mp}, \quad \mathrm{EI}-\mathrm{MS}, \quad{ }^{1} \mathrm{H}-, \\
{ }^{13} \mathrm{C}-\mathrm{NMR}\end{array}$ & $\begin{array}{l}\text { cytotoxic against five tumor } \\
\text { cell lines }\left(\mathrm{EC}_{50} 10-16 \mathrm{mM}\right)\end{array}$ & 13 \\
\hline 2002 & Duranta repens & 0.00002 & $\mathrm{n} / \mathrm{a}$ & $\begin{array}{l}\text { no inhibition of prolyl en- } \\
\text { dopeptidase }\end{array}$ & 43 \\
\hline 2002 & Ajuga remota & $\mathrm{n} / \mathrm{a}$ & $\mathrm{mp},[\alpha]_{\mathrm{D}}$ & $\begin{array}{l}\text { antiplasmodial activity } \\
\left(\mathrm{IC}_{50} \quad 8.2 \pm 1.1 \mu \mathrm{M}\right), \text { no } \\
\text { cytotoxicity at tested an- } \\
\text { tiplasmodic dose levels }\end{array}$ & 44 \\
\hline 2003 & G. annulare & $\mathrm{n} / \mathrm{a}$ & $\mathrm{n} / \mathrm{a}$ & $\begin{array}{l}\text { no antifungal activity } \\
\text { against Microsporum cannis } \\
\text { and Trichophyton mentagro- } \\
\text { phytes }\end{array}$ & 28 \\
\hline 2003 & Cordyceps cicadae & 0.0002 & $\mathrm{n} / \mathrm{a}$ & $\begin{array}{l}\text { immunosuppressive activity } \\
\text { by inhibition of activation } \\
\text { and various proliferation sig- } \\
\text { nals in primary human } \mathrm{T} \\
\text { lymphocytes }\end{array}$ & 61 \\
\hline 2004 & Poria cocos & 0.0007 & $\mathrm{mp},[\alpha]_{\mathrm{D}}$ & $\begin{array}{l}\text { moderate cytotoxicity } \\
\text { against the HT- } 29 \text { cell line } \\
\left(\mathrm{IC}_{50} 47 \mu \mathrm{M}\right) \text {, no inhibition } \\
\text { of topoisomerases I or II }\end{array}$ & 62 \\
\hline
\end{tabular}

Table 3-7 Literature $5 \alpha, 8 \alpha$-Epidioxyergosta-6,22-dien-3 $\beta$-ol

Occurrence in Ganodermataceae: G. amboinense, ${ }^{63}$ G. annulare ${ }^{28}$ G. applanatum,${ }^{27,64,65}$ G. australe ${ }^{30-32}$ G. carnosum,${ }^{33}$ G. concinna,${ }^{7}$ G. lucidum $;^{5,22,34,39,66-68}$ linoleoylester of $(6)^{53}$ 


\subsection{Ganoderol B / Ganodermadiol}

Isolation see Figure 2-1 on page 2. yield $8.0 \mathrm{mg}$ (min. $0.0037 \%$ in dried fruiting bodies of $G$. pfeifferi). TLC: $\mathrm{R}_{\mathrm{f}} 0.45$; fluorescence quenching at $254 \mathrm{~nm}$, no fluorescence at $366 \mathrm{~nm}$; violet with anisaldehyde-sulphuric acid reagent.

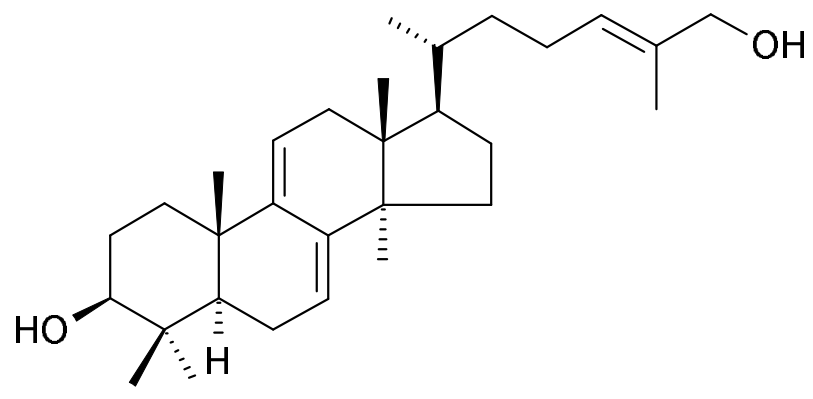

Ganoderol B

\subsubsection{UV-vis}

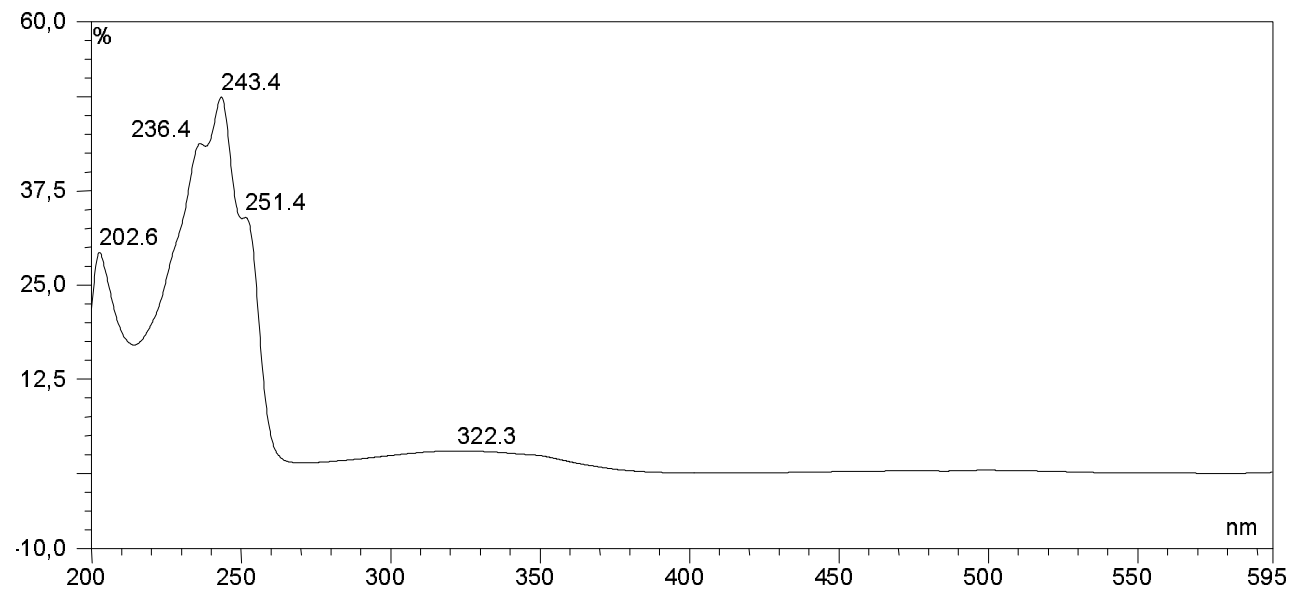

Figure 3-43 UV-vis Ganoderol B 


\subsubsection{EI-MS}

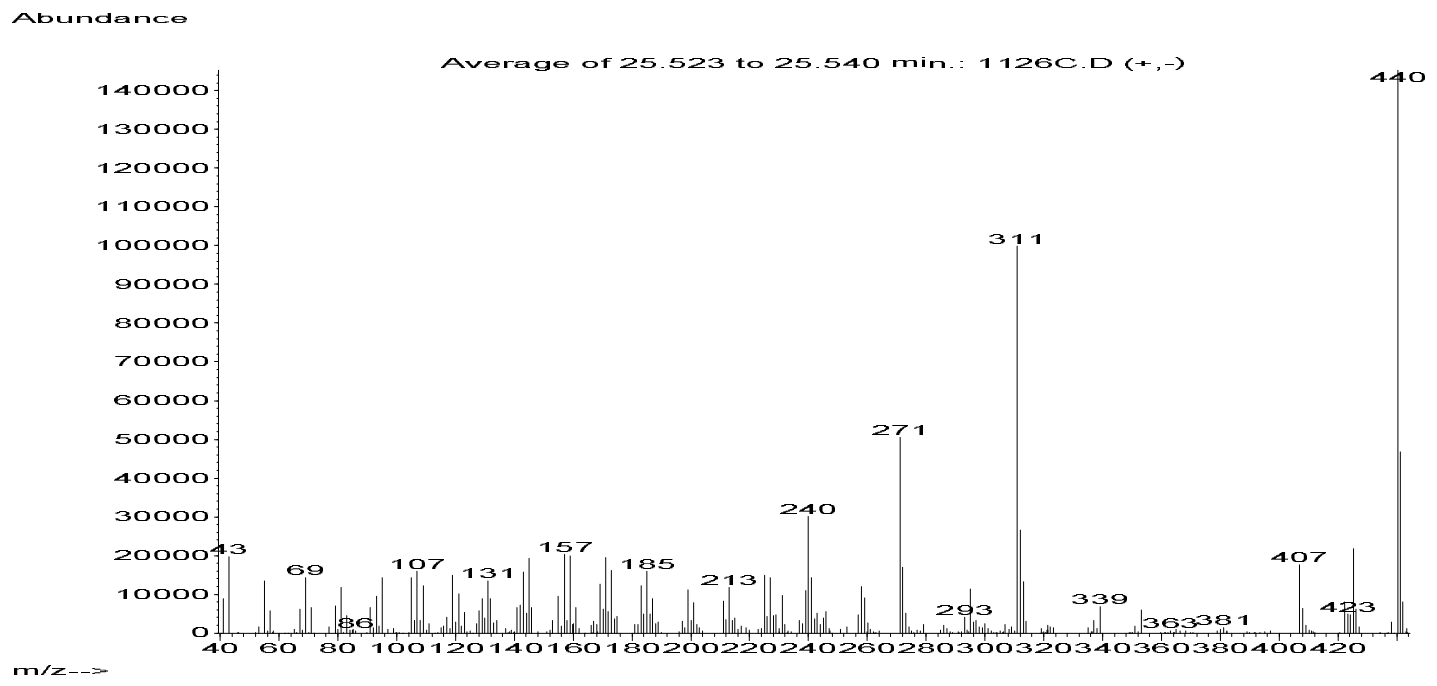

Figure 3-44 EI-MS Ganoderol B

\subsection{3 ${ }^{1} \mathrm{H}-\mathrm{NMR}$}

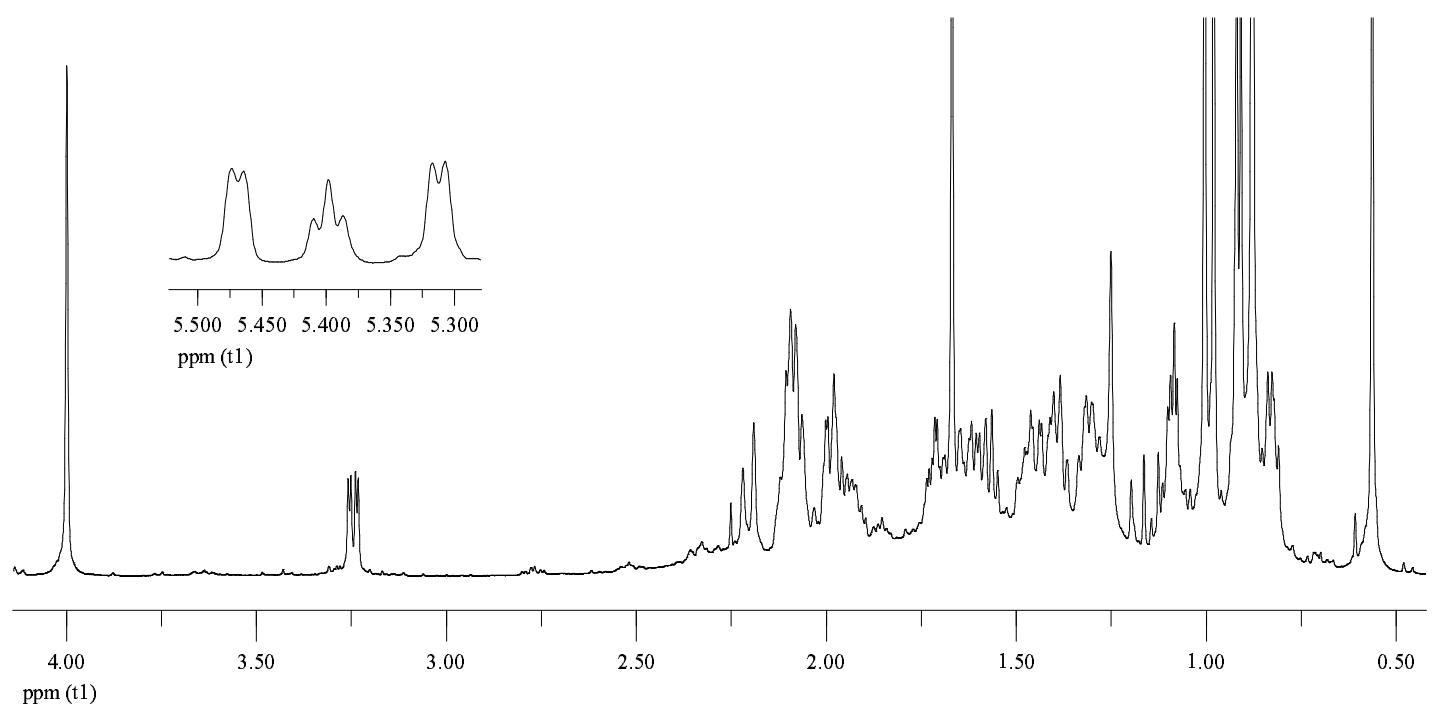

Figure 3-45 ${ }^{1} \mathrm{H}-\mathrm{NMR}$ Ganoderol $\mathrm{B}$ in $\mathrm{CDCl}_{3}$ 


\subsection{4 ${ }^{13} \mathrm{C}-\mathrm{NMR}$}

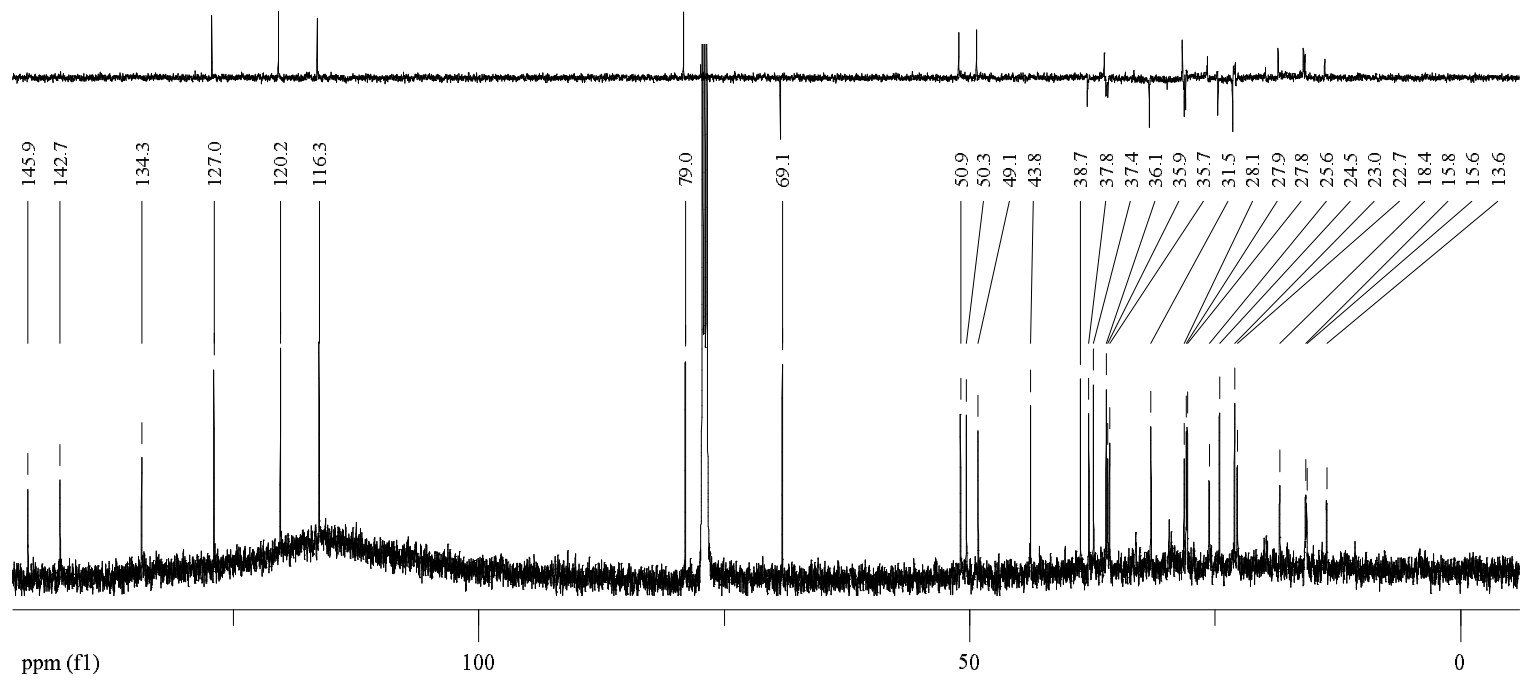

Figure 3-46 ${ }^{13} \mathrm{C}-\mathrm{NMR}$ and DEPT135 Ganoderol B in $\mathrm{CDCl}_{3}$

\subsubsection{Literature}

\begin{tabular}{|c|c|c|c|c|c|}
\hline year & isolated from & yield $(\%)$ & structure analysis & biol. activity & in \\
\hline 1986 & G. lucidum & $\mathrm{n} / \mathrm{a}$ & $\begin{array}{l}\mathrm{mp}, \quad[\alpha]_{\mathrm{D}}, \quad \mathrm{UV}-\text {-vis, } \\
\mathrm{R}_{\mathrm{f}}, \text { EI-MS, }{ }^{1} \mathrm{H}-\mathrm{NMR}\end{array}$ & $\begin{array}{l}\text { inhibition of Angiotensin } \\
\text { Converting Enzyme }\left(\mathrm{IC}_{50}\right. \\
\left.2.2 \times 10^{-4} \mathrm{M}\right)\end{array}$ & 8 \\
\hline 1986 & G. lucidum & 0.0004 & 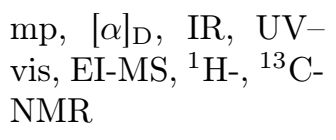 & $\mathrm{n} / \mathrm{a}$ & 39 \\
\hline 1987 & G. lucidum & $\mathrm{n} / \mathrm{a}$ & EI-MS, IR, ${ }^{1} \mathrm{H}-\mathrm{NMR}$ & $\mathrm{n} / \mathrm{a}$ & 9 \\
\hline 1988 & G. lucidum & $\mathrm{n} / \mathrm{a}$ & {$[\alpha]_{\mathrm{D}}, \mathrm{UV}-\mathrm{vis}$} & $\mathrm{n} / \mathrm{a}$ & 36 \\
\hline 1988 & G. lucidum & $\mathrm{n} / \mathrm{a}$ & ${ }^{1} \mathrm{H}-,{ }^{13} \mathrm{C}-\mathrm{NMR}$ & $\mathrm{n} / \mathrm{a}$ & 40 \\
\hline 1998 & G. neo-japonicum & 0.0055 & $\mathrm{n} / \mathrm{a}$ & $\mathrm{n} / \mathrm{a}$ & 6 \\
\hline 2002 & G. lucidum & 0.0127 & $\mathrm{n} / \mathrm{a}$ & $\begin{array}{l}\text { cytotoxicity against four tu- } \\
\text { mor cell lines }\left(\mathrm{EC}_{50} 10->20\right. \\
\mu \mathrm{g} / \mathrm{mL})\end{array}$ & 37 \\
\hline 2002 & G. concinna & 0.0176 & $\mathrm{n} / \mathrm{a}$ & $\mathrm{n} / \mathrm{a}$ & 7 \\
\hline 2003 & G. pfeifferi & 0.035 & $\begin{array}{l}\text { mp, UV-vis, IR, EI- } \\
\text { MS }\end{array}$ & $\begin{array}{l}\text { antiviral activity against in- } \\
\text { fluenza virus A }\left(\mathrm{ED}_{50}>0.22\right. \\
\mathrm{mmol} / \mathrm{L}) \text { and } \mathrm{HSV} 1\left(\mathrm{ED}_{50}\right. \\
0.068 \mathrm{mmol} / \mathrm{L})\end{array}$ & 69 \\
\hline
\end{tabular}

Table 3-8 Literature Ganoderol B 


\subsection{Applanoxidic acid C}

Isolation see Figure 2-1 on page 2. white flakes $(\mathrm{MeOH}$ ). yield $38.4 \mathrm{mg}$ (min. $0.0177 \%$ in dried fruiting bodies of $G$. pfeifferi). TLC: $\mathrm{R}_{\mathrm{f}} 0.31$; fluorescence quenching at $254 \mathrm{~nm}$, no fluorescence at $366 \mathrm{~nm}$; weak orange with anisaldehyde-sulphuric acid reagent.

HRFTICRMS [M+H] ${ }^{+}$: calcd for $\mathrm{C}_{30} \mathrm{H}_{39} \mathrm{O}_{8}$ : 527.2645; found: 527.2603 .

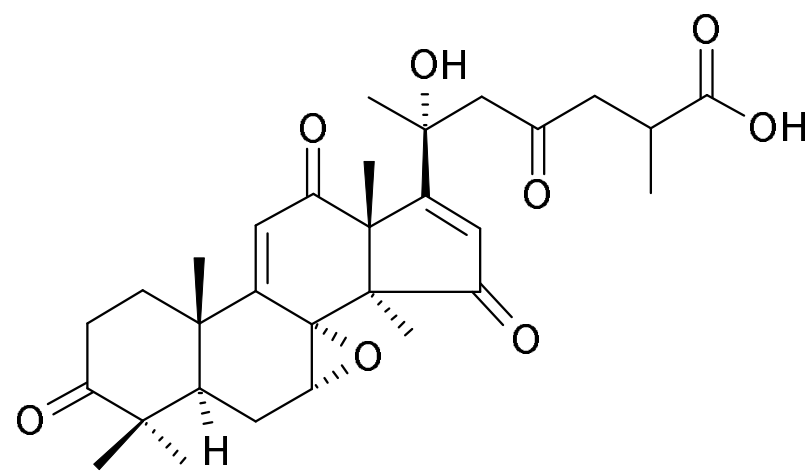

Applanoxidic acid C

\subsubsection{UV-vis}

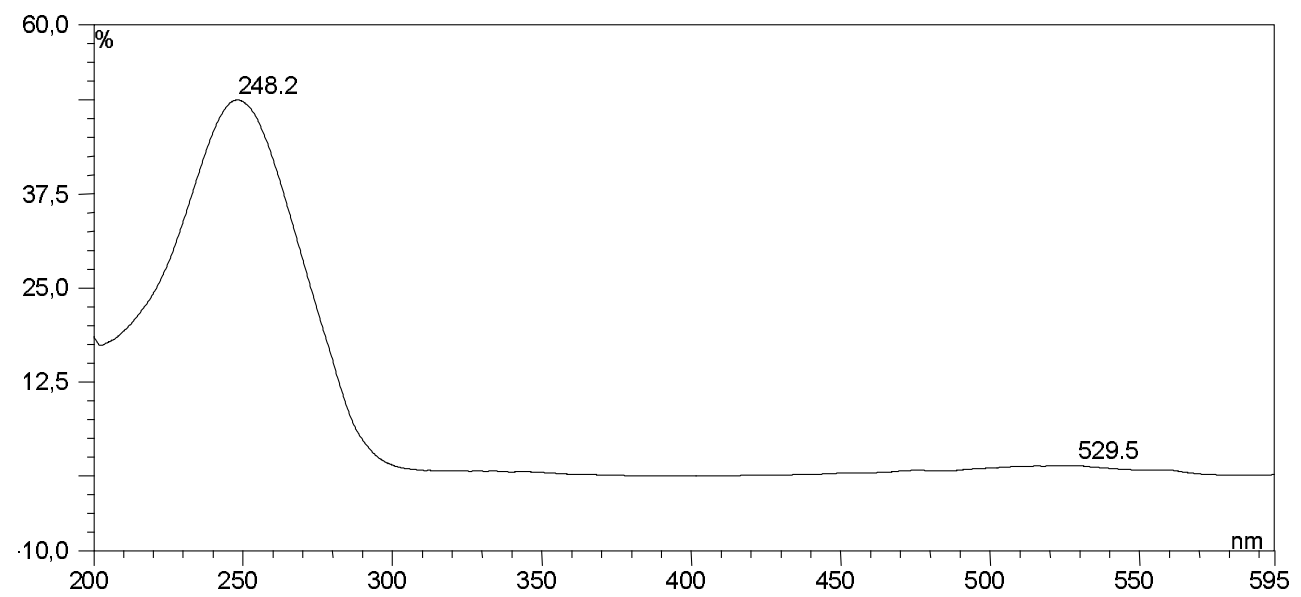

Figure 3-47 UV-vis Applanoxidic acid C 


\subsubsection{EI-MS}

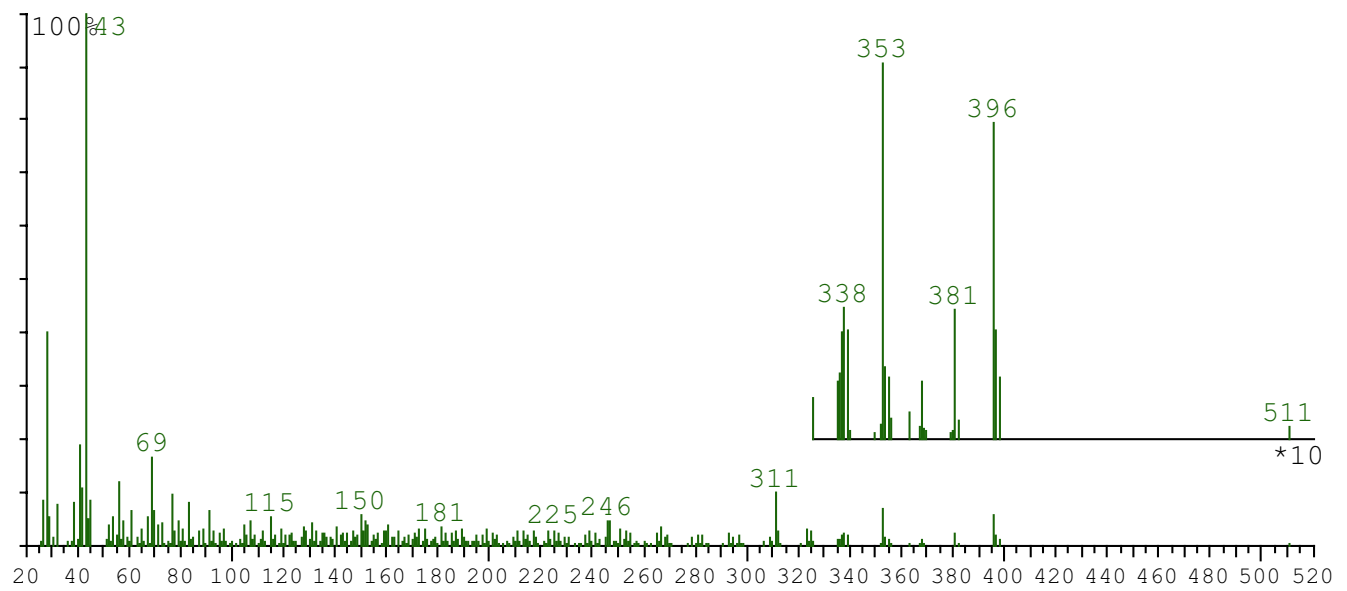

Figure 3-48 EI-MS Applanoxidic acid C

\subsection{3 ${ }^{1} \mathrm{H}-\mathrm{NMR}$}
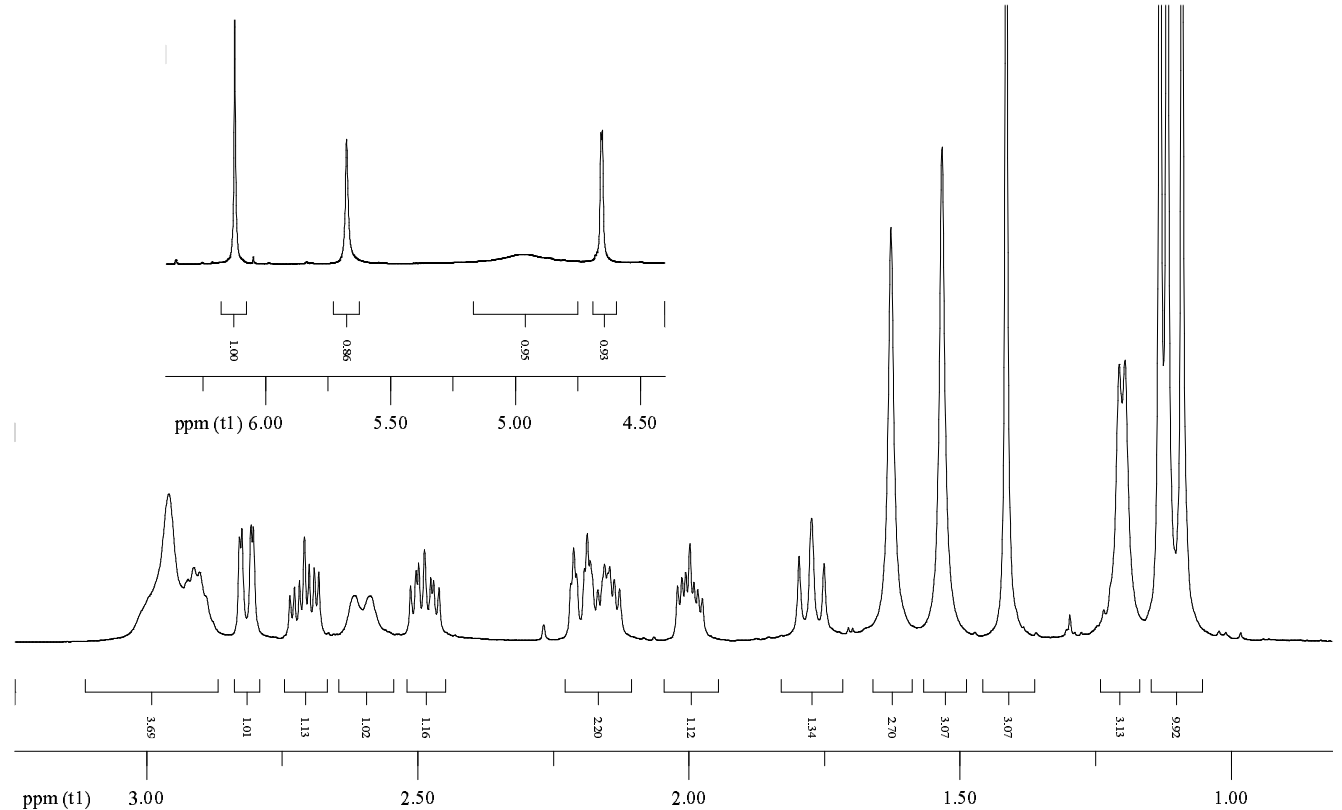

Figure 3-49 ${ }^{1} \mathrm{H}-\mathrm{NMR}$ Applanoxidic acid $\mathrm{C}$ in $\mathrm{CDCl}_{3}$ 


\subsection{4 ${ }^{13} \mathrm{C}-\mathrm{NMR}$}

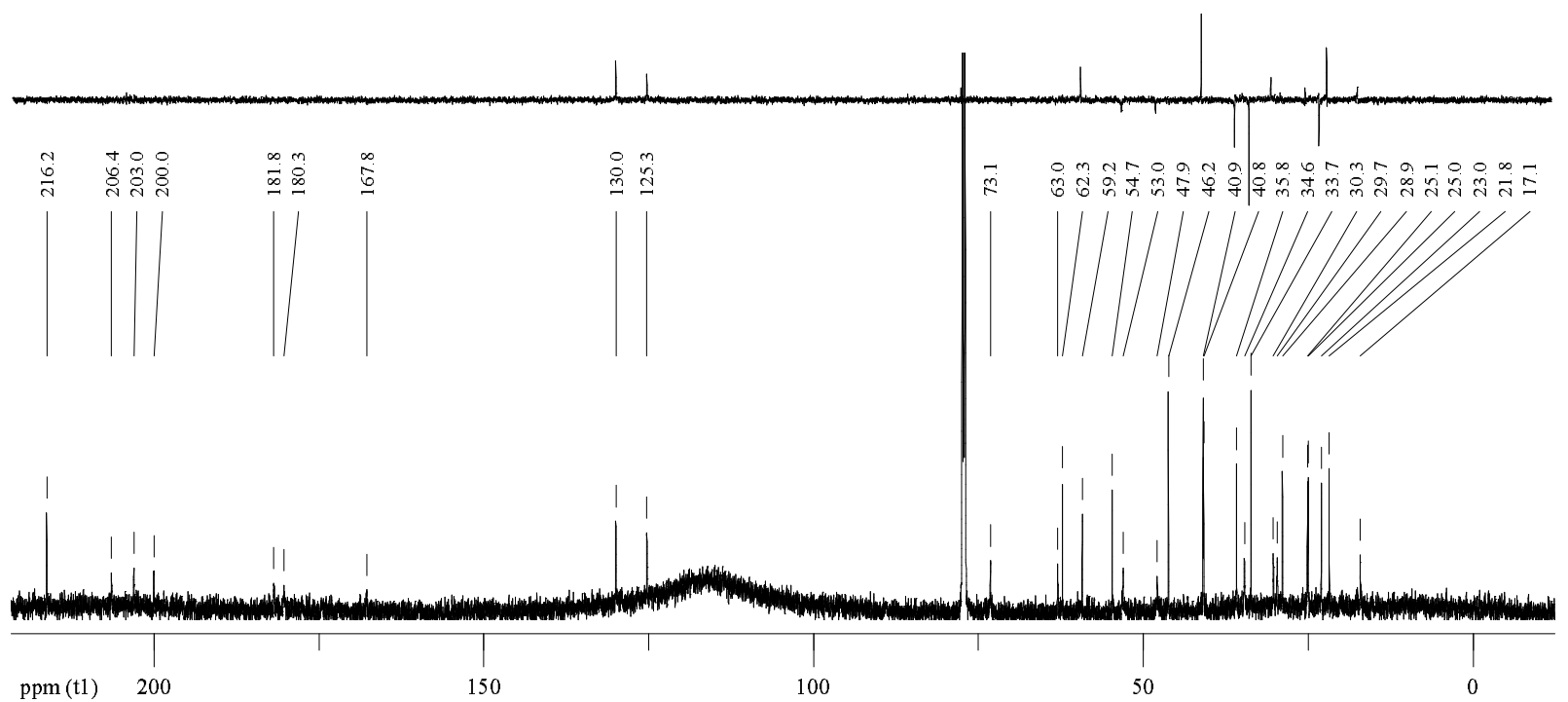

Figure 3-50 ${ }^{13} \mathrm{C}-\mathrm{NMR}$ and DEPT135 Applanoxidic acid $\mathrm{C}$ in $\mathrm{CDCl}_{3}$

\subsubsection{Literature}

\begin{tabular}{|c|c|c|c|c|c|}
\hline year & isolated from & yield $(\%)$ & structure analysis & biol. activity & in \\
\hline 1991 & G. applanatum & $\mathrm{n} / \mathrm{a}$ & $\begin{array}{l}\mathrm{mp},[\alpha]_{\mathrm{D}}, \mathrm{UV}-\text { vis, IR, } \\
\text { EI-MS, }{ }^{1} \mathrm{H}-,{ }^{13} \mathrm{C}-\mathrm{NMR}\end{array}$ & $\begin{array}{l}\text { inhibitory effect on Epstein-Barr } \\
\text { virus-associated early antigen ac- } \\
\text { tivation (mouse skin tumor pro- } \\
\text { moter) }\end{array}$ & 70 \\
\hline 2000 & G. australe & $\mathrm{n} / \mathrm{a}$ & $\mathrm{n} / \mathrm{a}$ & $\mathrm{n} / \mathrm{a}$ & 31 \\
\hline 2003 & G. annulare & $\mathrm{n} / \mathrm{a}$ & $\mathrm{n} / \mathrm{a}$ & $\begin{array}{l}\text { very weak antifungal activity } \\
\text { against Microsporum cannis and } \\
\text { Trichophyton mentagrophytes } \\
\text { (MIC } 1000 \mu \mathrm{g} / \mathrm{mL} \text { ) }\end{array}$ & 28 \\
\hline 2003 & G. australe & 0.0009 & $\mathrm{n} / \mathrm{a}$ & $\begin{array}{l}\text { cytotoxic for HL-60 cells }\left(\mathrm{IC}_{50}\right. \\
334 \pm 90 \mu \mathrm{M})\end{array}$ & 30 \\
\hline
\end{tabular}

Table 3-9 Literature Applanoxidic acid C 


\subsection{Ganoderone A *}

Isolation see Figure 2-1 on page 2. white needles $(\mathrm{MeOH})$. yield $23.8 \mathrm{mg}$ (min. $0.0246 \%$ in dried fruiting bodies of $G$. pfeifferi). TLC: $\mathrm{R}_{\mathrm{f}} 0.35$; fluorescence quenching at $254 \mathrm{~nm}$, no fluorescence at $366 \mathrm{~nm}$; violet with anisaldehyde-sulphuric acid reagent.

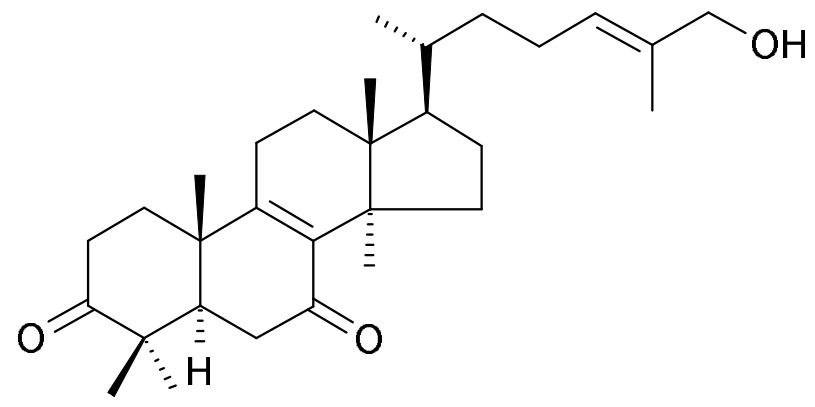

(2) Ganoderone A

\subsubsection{UV-vis}

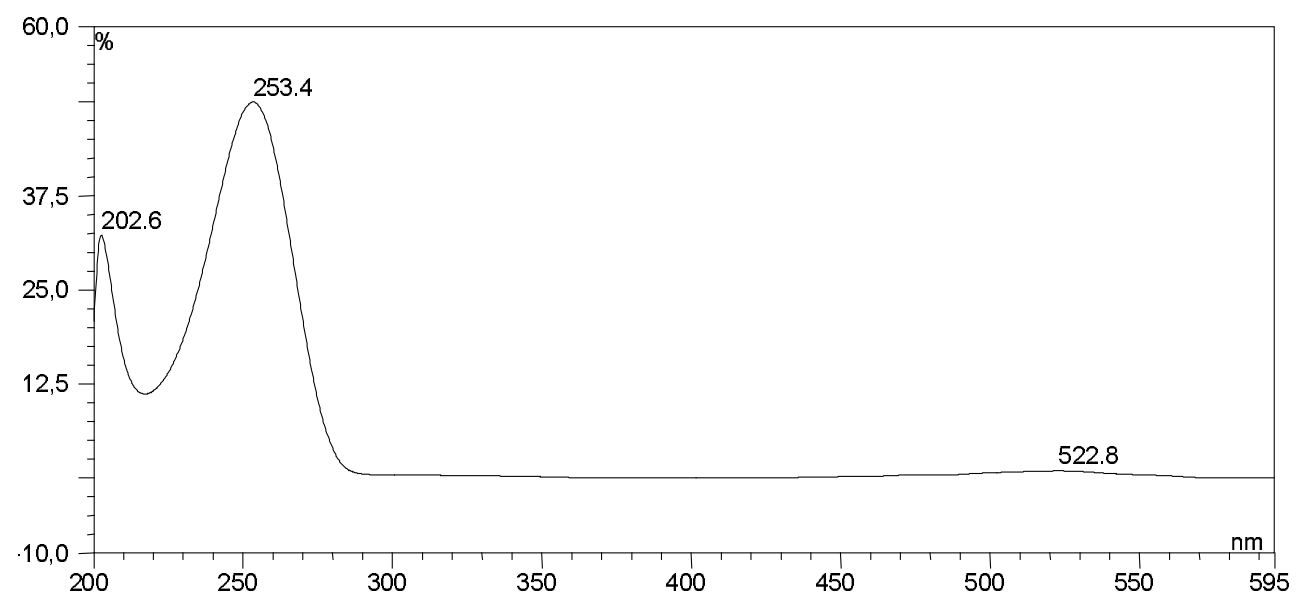

Figure 3-51 UV-vis Ganoderone A 


\subsubsection{EI-MS}

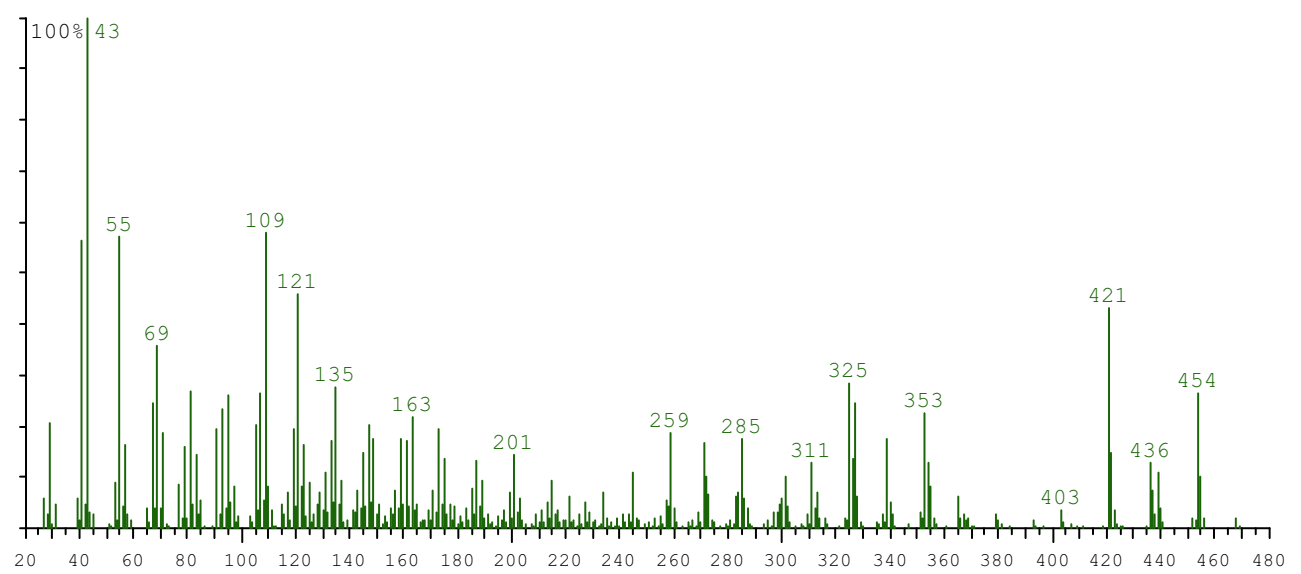

Figure 3-52 EI-MS Ganoderone A

(loss of $\mathrm{H}_{2} \mathrm{O}$ in GC-MS $\rightarrow$ only fragment with $\mathrm{M}_{\mathrm{r}} 436$ detectable).

\subsubsection{IR}

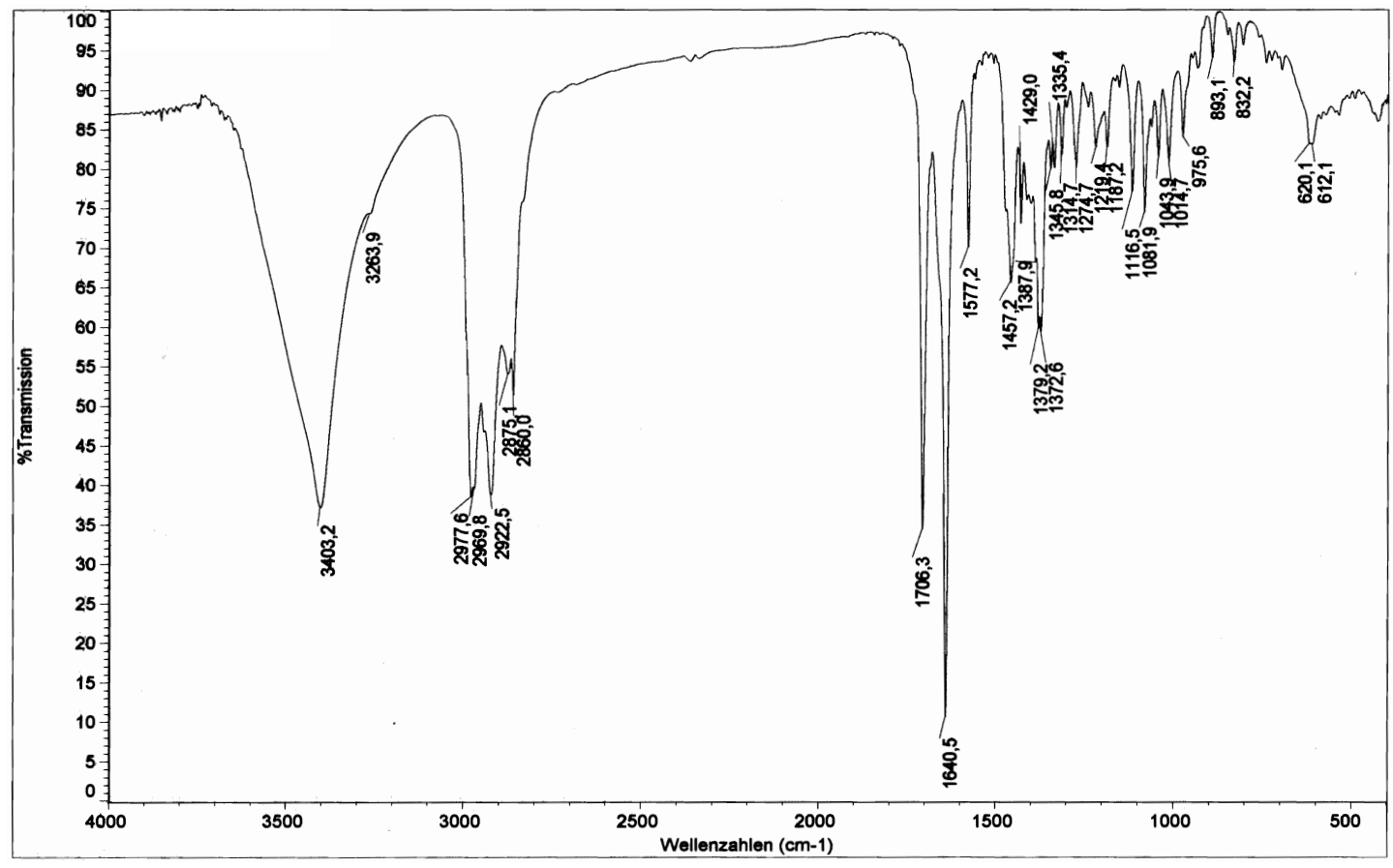

Figure 3-53 IR Ganoderone A 


\subsection{4 ${ }^{1} \mathrm{H}-\mathrm{NMR}$}

\subsubsection{1 ${ }^{1} \mathrm{H}-\mathrm{NMR}$ overview}

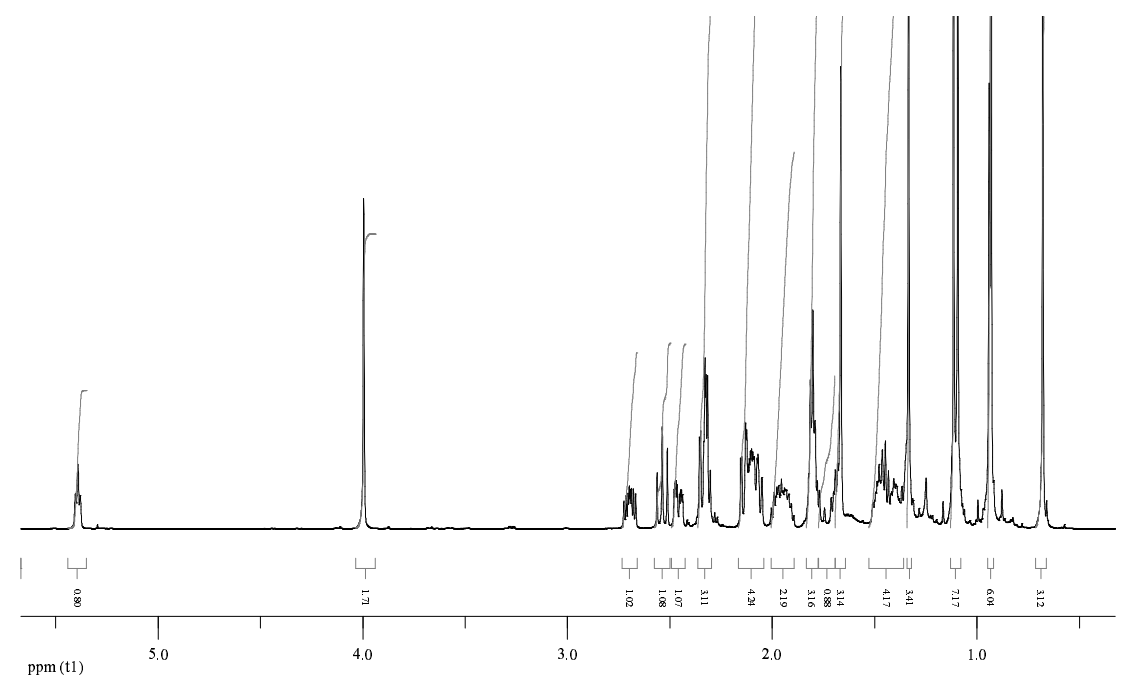

Figure 3-54 ${ }^{1} \mathrm{H}-\mathrm{NMR}$ Ganoderone $\mathrm{A}$ in $\mathrm{CDCl}_{3}$ (overview)

\subsubsection{2 ${ }^{1} \mathrm{H}-\mathrm{NMR}$ detail}

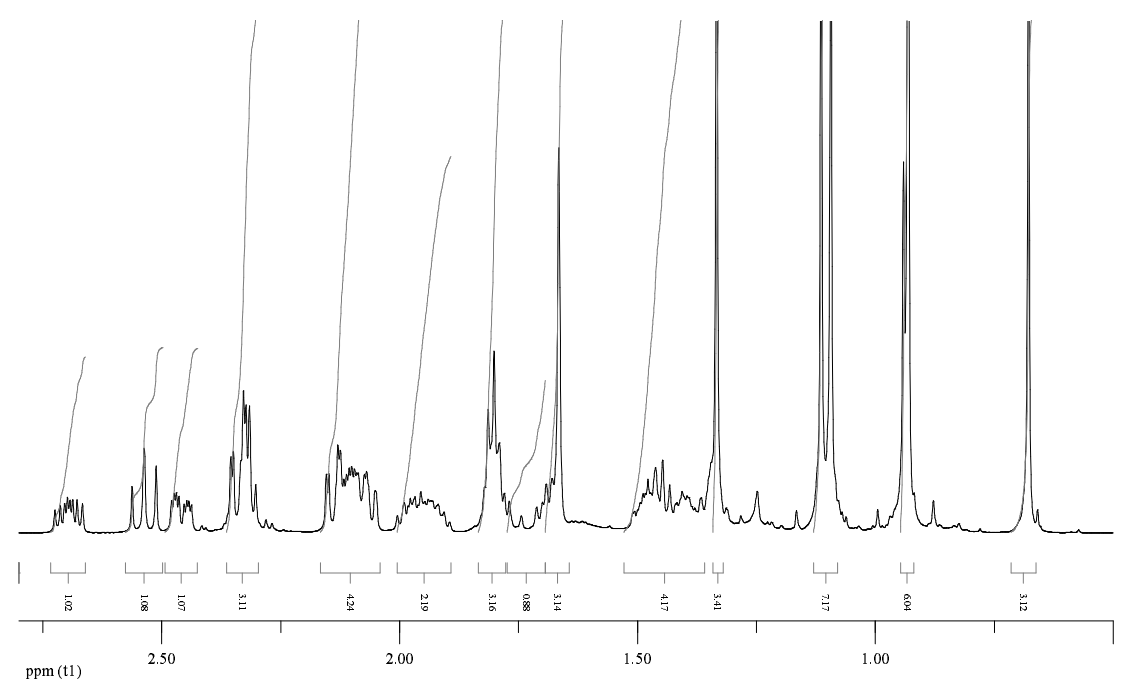

Figure 3-55 ${ }^{1} \mathrm{H}-\mathrm{NMR}$ Ganoderone $\mathrm{A}$ in $\mathrm{CDCl}_{3}$ (0.5 to $2.8 \mathrm{ppm}$ ) 


\subsection{5 ${ }^{13} \mathrm{C}-\mathrm{NMR}$}

\subsubsection{1 ${ }^{13} \mathrm{C}-\mathrm{NMR}$ overview}

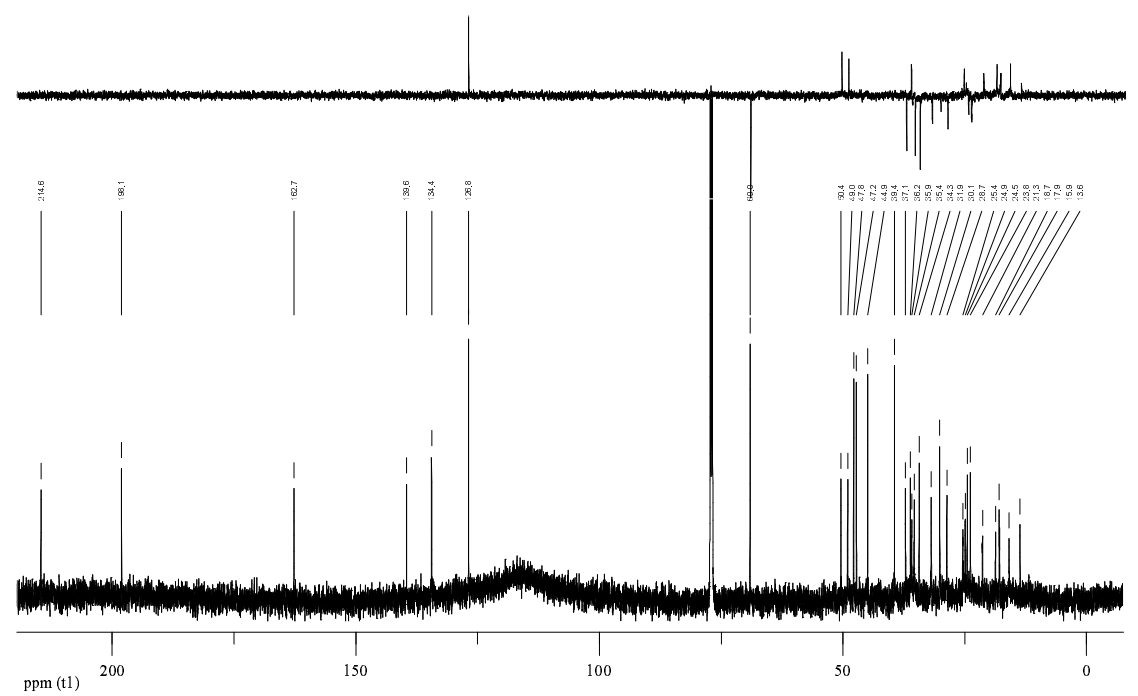

Figure 3-56 ${ }^{13} \mathrm{C}-\mathrm{NMR}$ and $\mathrm{DEPT} 135$ Ganoderone $\mathrm{A}$ in $\mathrm{CDCl}_{3}$ (overview)

\subsubsection{2 ${ }^{13} \mathrm{C}-\mathrm{NMR}$ detail}

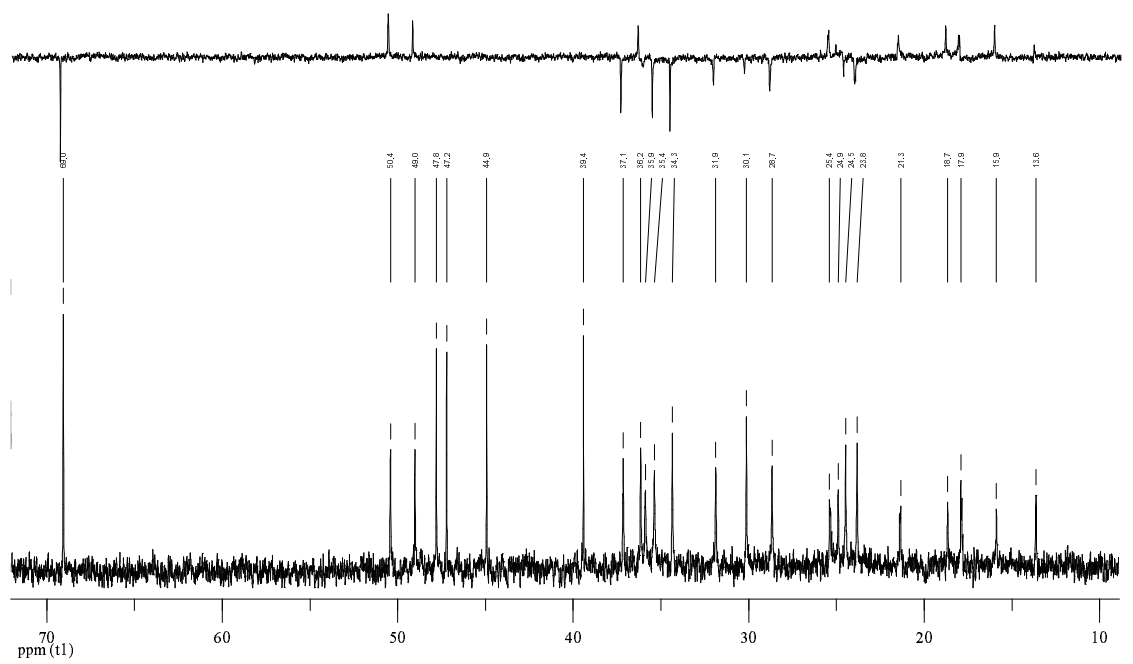

Figure 3-57 ${ }^{13} \mathrm{C}-\mathrm{NMR}$ and DEPT135 Ganoderone $\mathrm{A}$ in $\mathrm{CDCl}_{3}$ (9 to $72 \mathrm{ppm}$ ) 


\subsubsection{HSQC}

\subsubsection{HSQC overview}

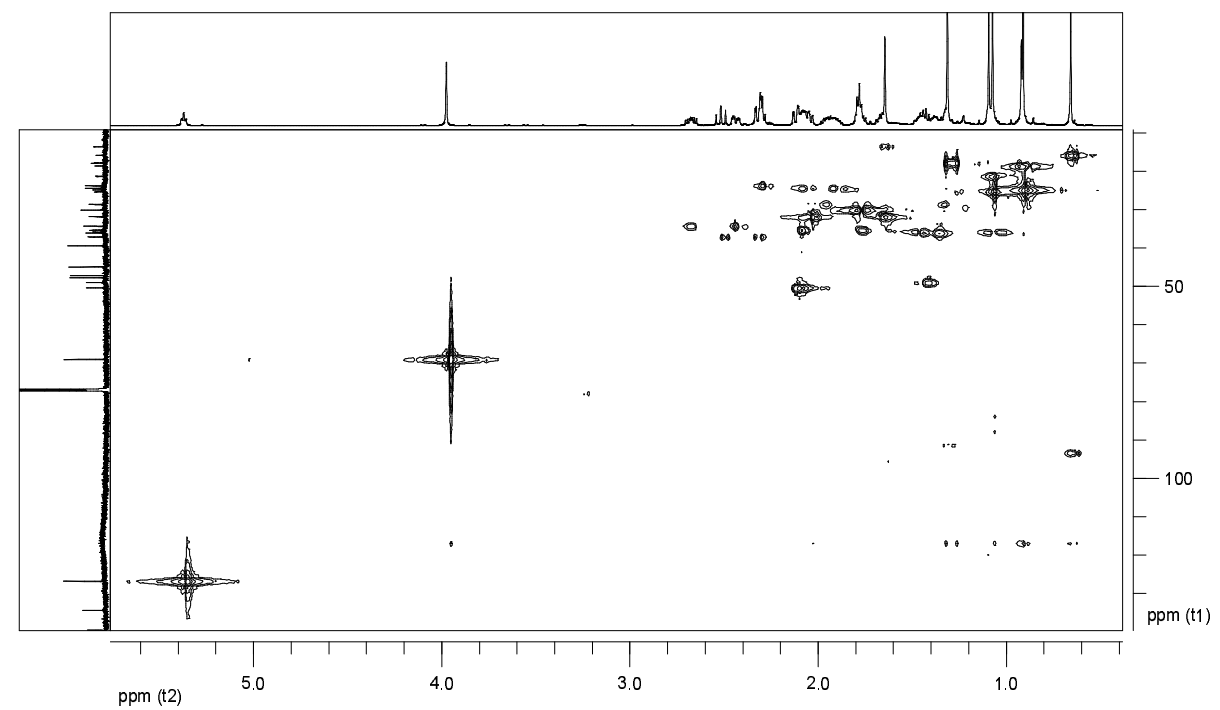

Figure 3-58 HSQC Ganoderone $\mathrm{A}$ in $\mathrm{CDCl}_{3}\left(0.4\right.$ to $5.7\left({ }^{1} \mathrm{H}\right), 9$ to $\left.140 \mathrm{ppm}\left({ }^{13} \mathrm{C}\right)\right)$

\subsubsection{HSQC detail}

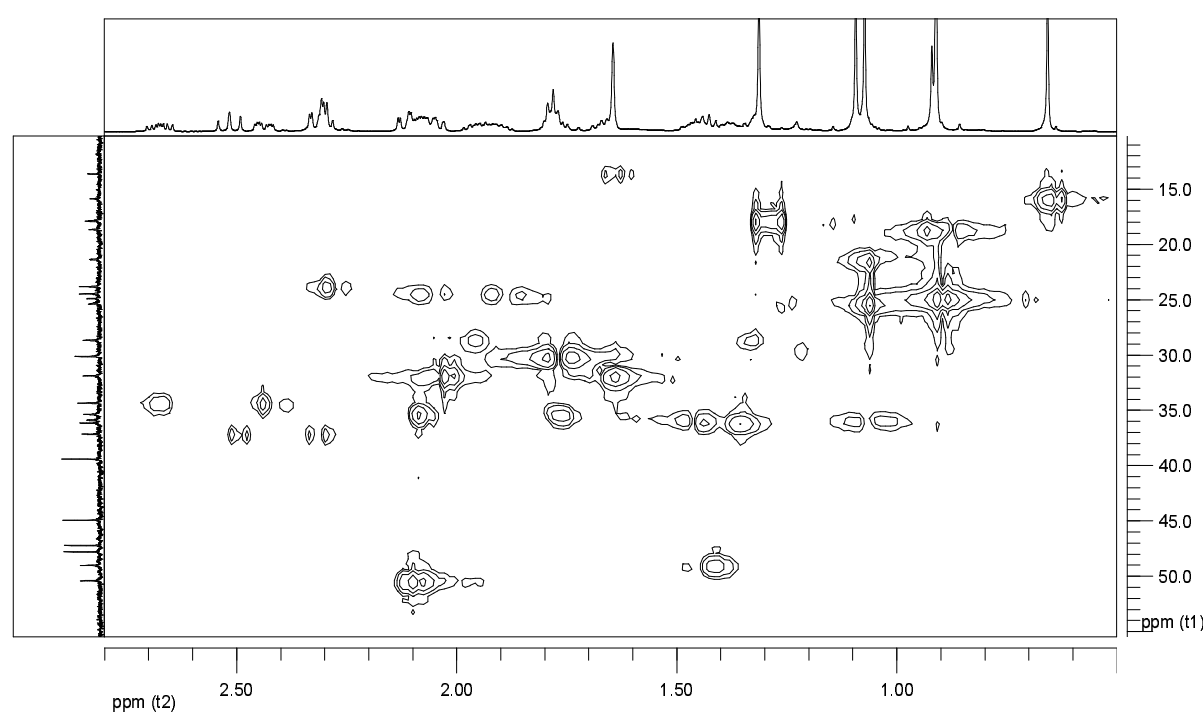

Figure 3-59 HSQC Ganoderone $\mathrm{A}$ in $\mathrm{CDCl}_{3}\left(0.5\right.$ to $2.8\left({ }^{1} \mathrm{H}\right), 10$ to $\left.55 \mathrm{ppm}\left({ }^{13} \mathrm{C}\right)\right)$ 


\subsection{7 $\mathrm{HMBC}$}

\subsubsection{HMBC overview}

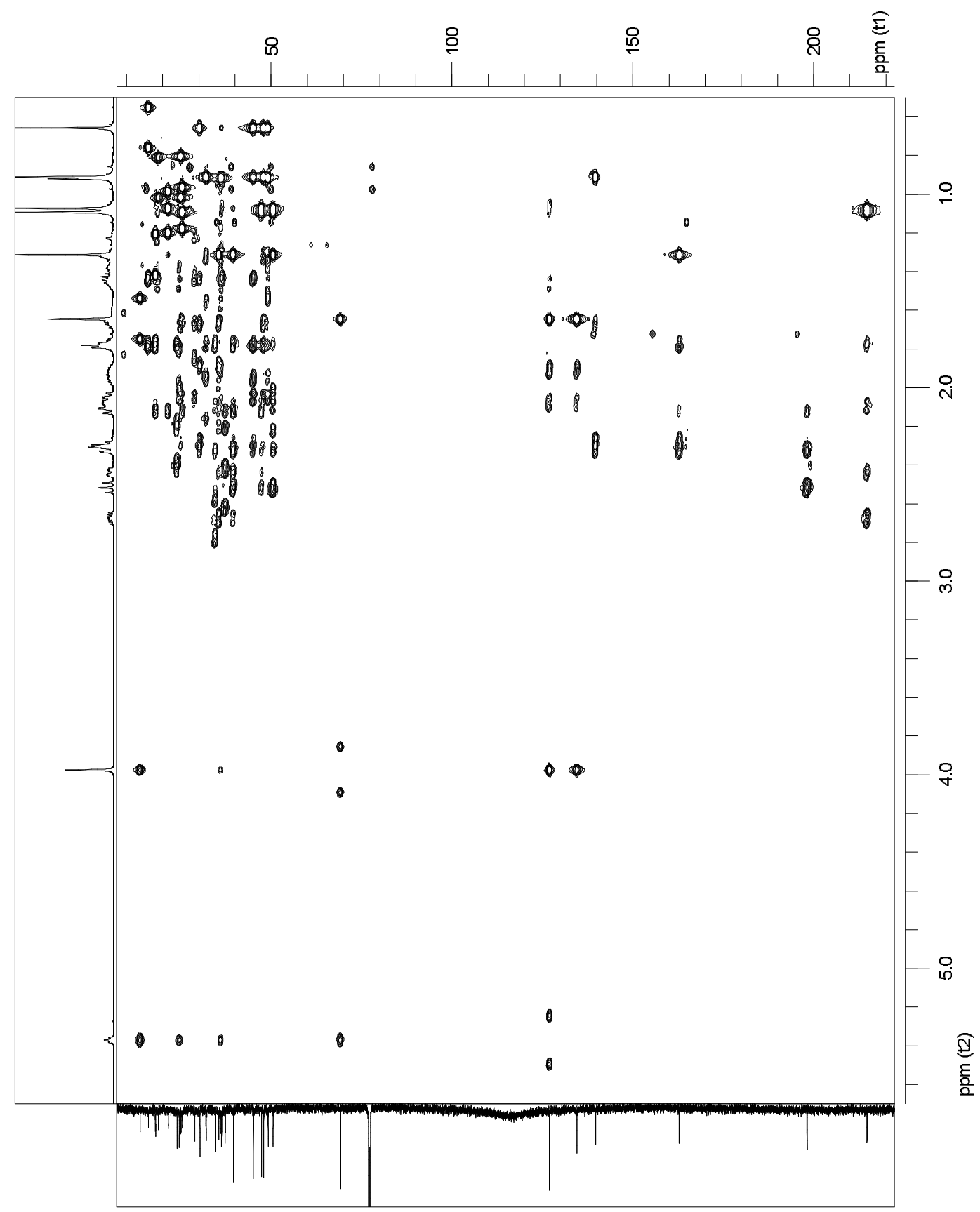

Figure 3-60 HMBC Ganoderone $\mathrm{A}$ in $\mathrm{CDCl}_{3}\left(0.5\right.$ to $5.7\left({ }^{1} \mathrm{H}\right), 7$ to $\left.222 \mathrm{ppm}\left({ }^{13} \mathrm{C}\right)\right)$ 


\subsubsection{HMBC detail}

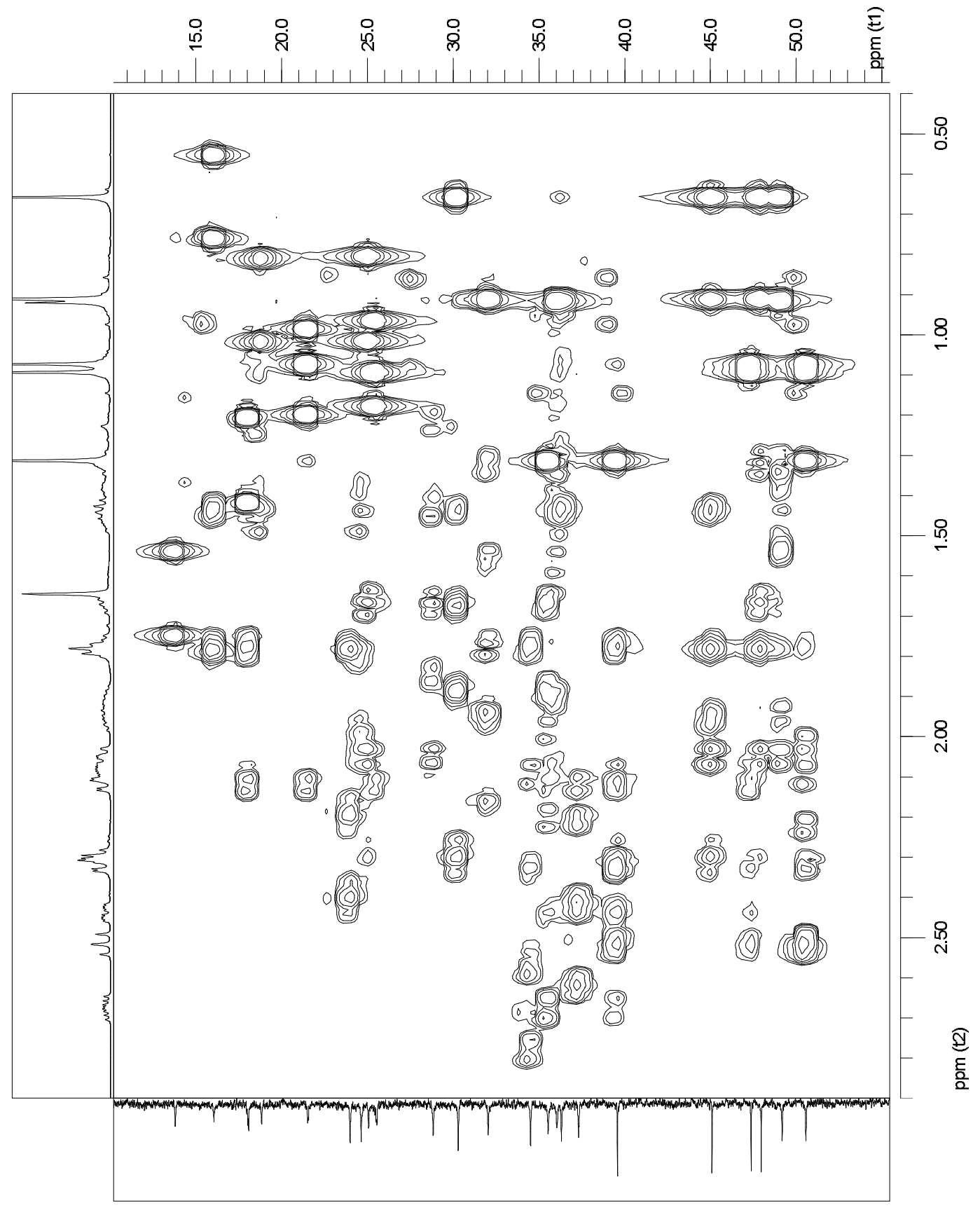

Figure 3-61 HMBC Ganoderone $\mathrm{A}$ in $\mathrm{CDCl}_{3}\left(0.4\right.$ to $2.9\left({ }^{1} \mathrm{H}\right), 10$ to $\left.55 \mathrm{ppm}\left({ }^{13} \mathrm{C}\right)\right)$ 


\subsubsection{HMBC correlations}

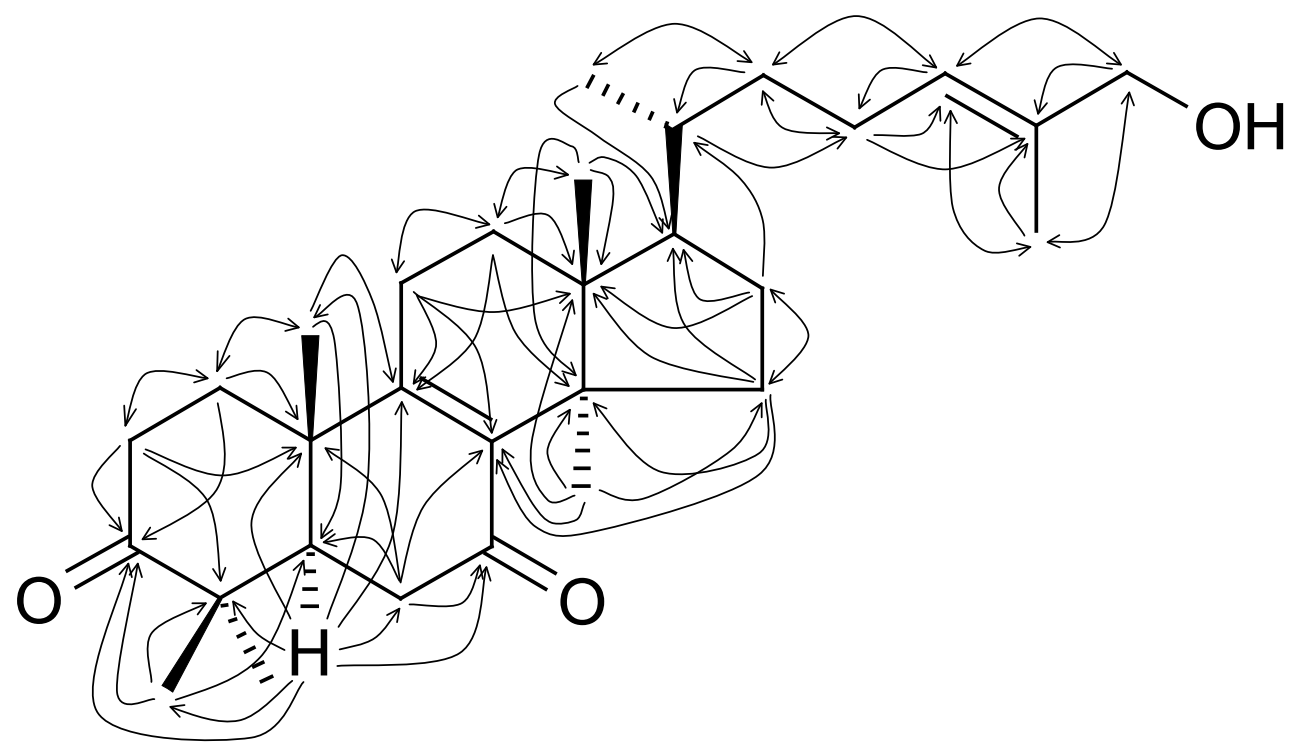

Figure 3-62 HMBC correlations Ganoderone A 


\subsubsection{NOESY}

\subsubsection{NOESY overview}

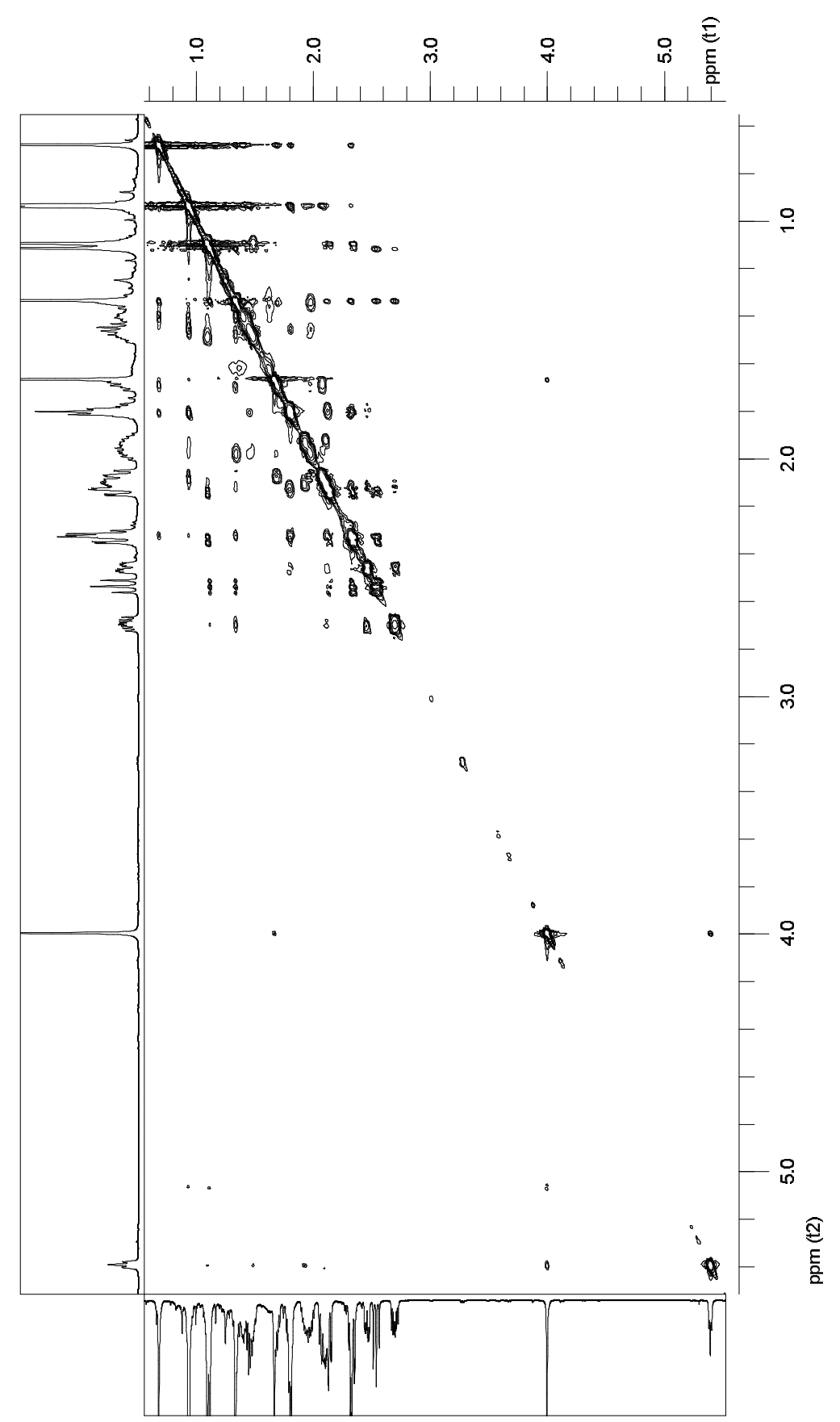

Figure 3-63 NOESY Ganoderone $\mathrm{A}$ in $\mathrm{CDCl}_{3}$ (overview) 


\subsubsection{NOESY detail}

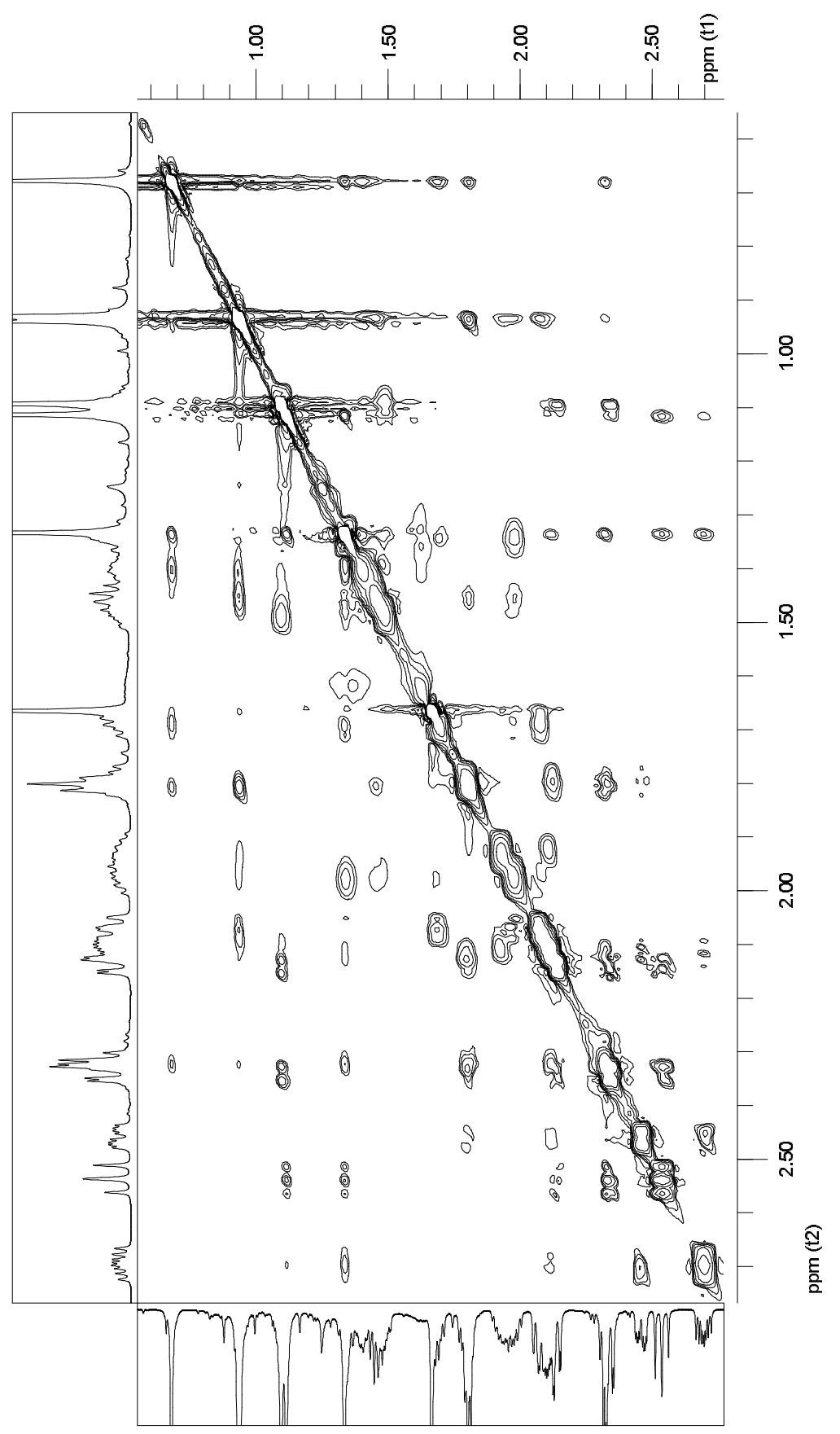

Figure 3-64 NOESY Ganoderone $\mathrm{A}$ in $\mathrm{CDCl}_{3}\left(0.55\right.$ to $2.77\left({ }^{1} \mathrm{H}\right)$ ) 


\subsection{9 similar natural compounds}

- Ganoderol A see section 3.7

- Ganoderone $\mathrm{B}^{22,69}$

- Ganoderone $\mathrm{C}$ this article

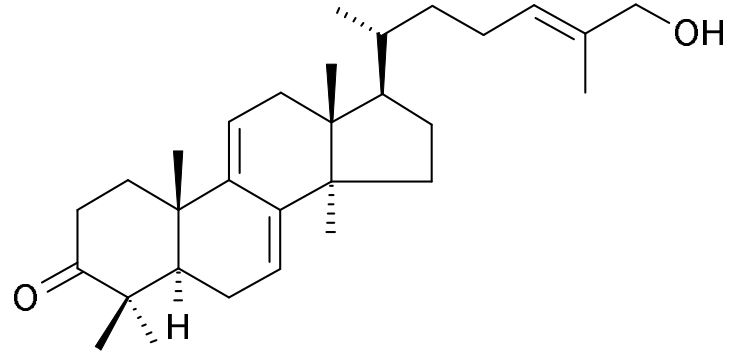

Figure 3-65 Ganoderol A

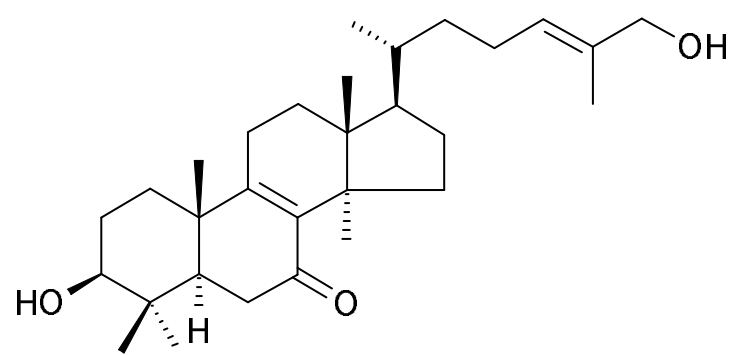

Figure 3-66 Ganoderone B<smiles>C[C@H](CC[C@@H]1O[C@]1(C)CO)[C@H]1CC[C@]2(C)C3=C(CC[C@]12C)[C@@]1(C)CCC(=O)[C@](C)(CO)[C@H]1CC3=O</smiles>

Figure 3-67 Ganoderone C 


\subsection{Ganoderone C *}

Isolation see Figure 2-1 on page 2. white amorphous powder. yield $13.7 \mathrm{mg}$ (min. $0.0144 \%$ in dried fruiting bodies of $G$. pfeifferi). TLC: $\mathrm{R}_{\mathrm{f}} 0.19$; fluorescence quenching at $254 \mathrm{~nm}$, no fluorescence at $366 \mathrm{~nm}$; weak orange with anisaldehyde-sulphuric acid reagent.

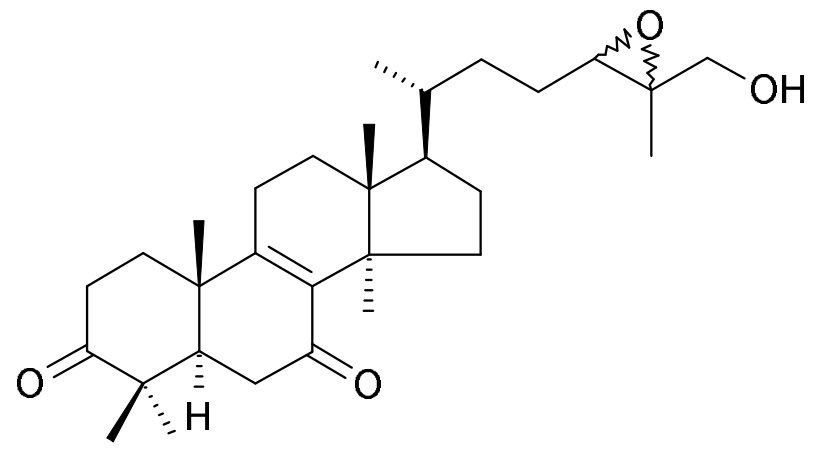

(3) Ganoderone C

\subsubsection{UV-vis}

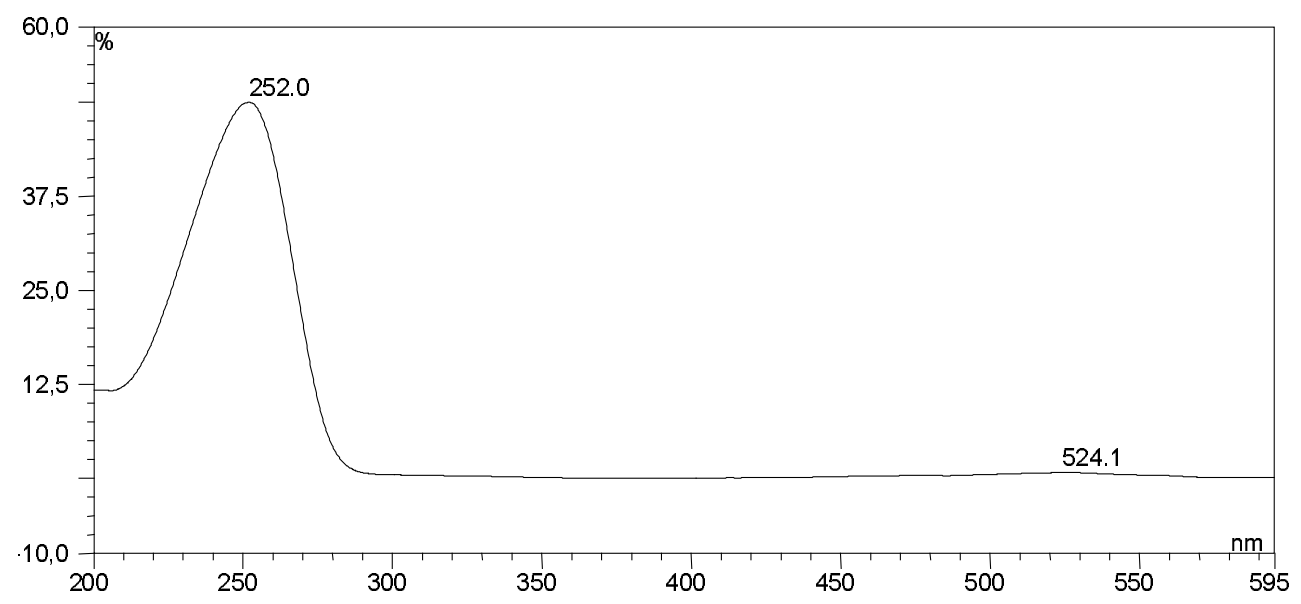

Figure 3-68 UV-vis Ganoderone C 


\subsubsection{EI-MS}

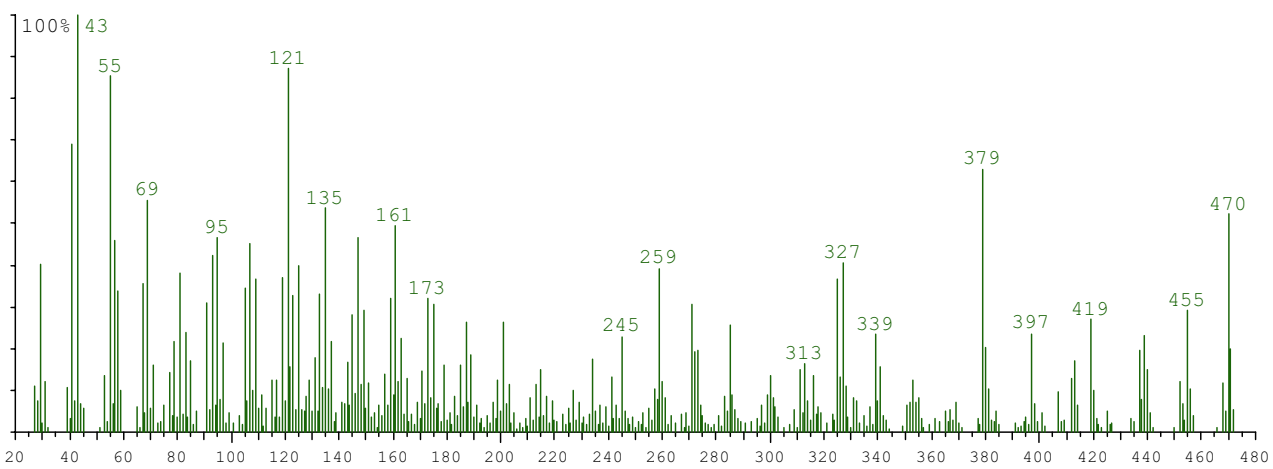

Figure 3-69 EI-MS Ganoderone C

Decomposition in GC-MS (only fragment with $\mathrm{M}_{\mathrm{r}} 412$ detectable).

\subsubsection{IR}

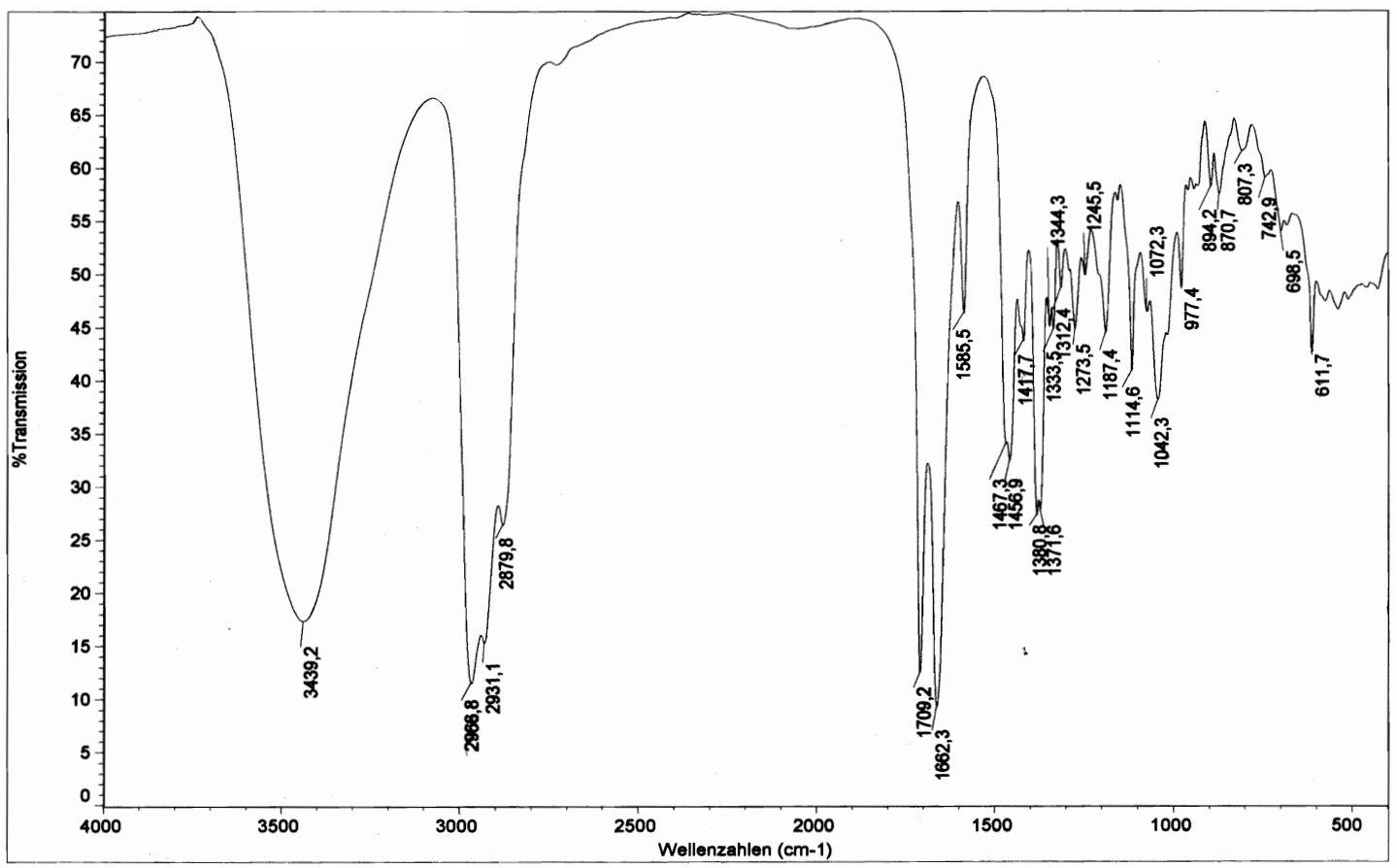

Figure 3-70 IR Ganoderone C 


\section{$3.12 .4{ }^{1} \mathrm{H}-\mathrm{NMR}$}

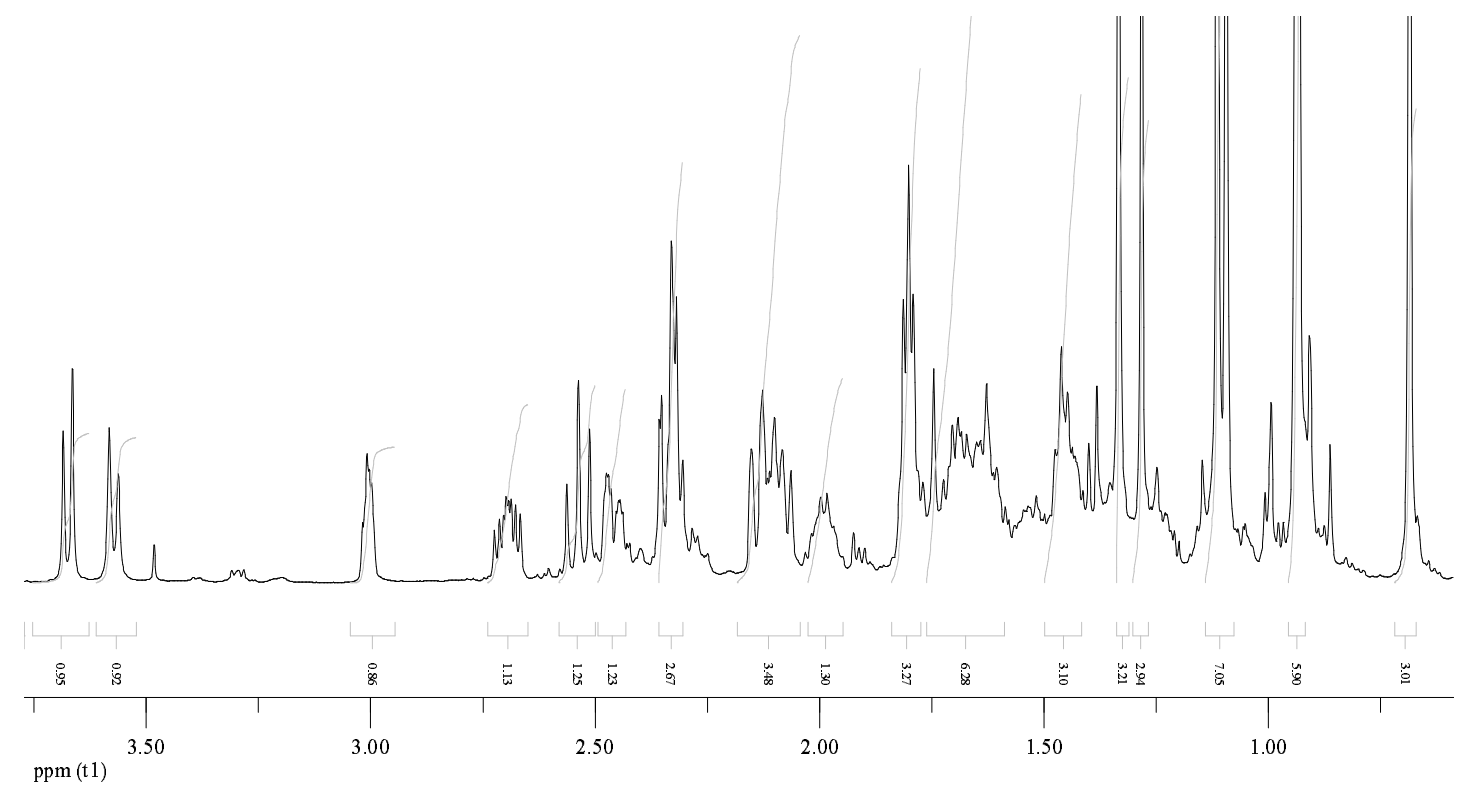

Figure 3-71 ${ }^{1} \mathrm{H}-\mathrm{NMR}$ Ganoderone $\mathrm{C}$ in $\mathrm{CDCl}_{3}$ 


\subsection{5 ${ }^{13} \mathrm{C}-\mathrm{NMR}$}

\subsubsection{1 ${ }^{13} \mathrm{C}-\mathrm{NMR}$ overview}

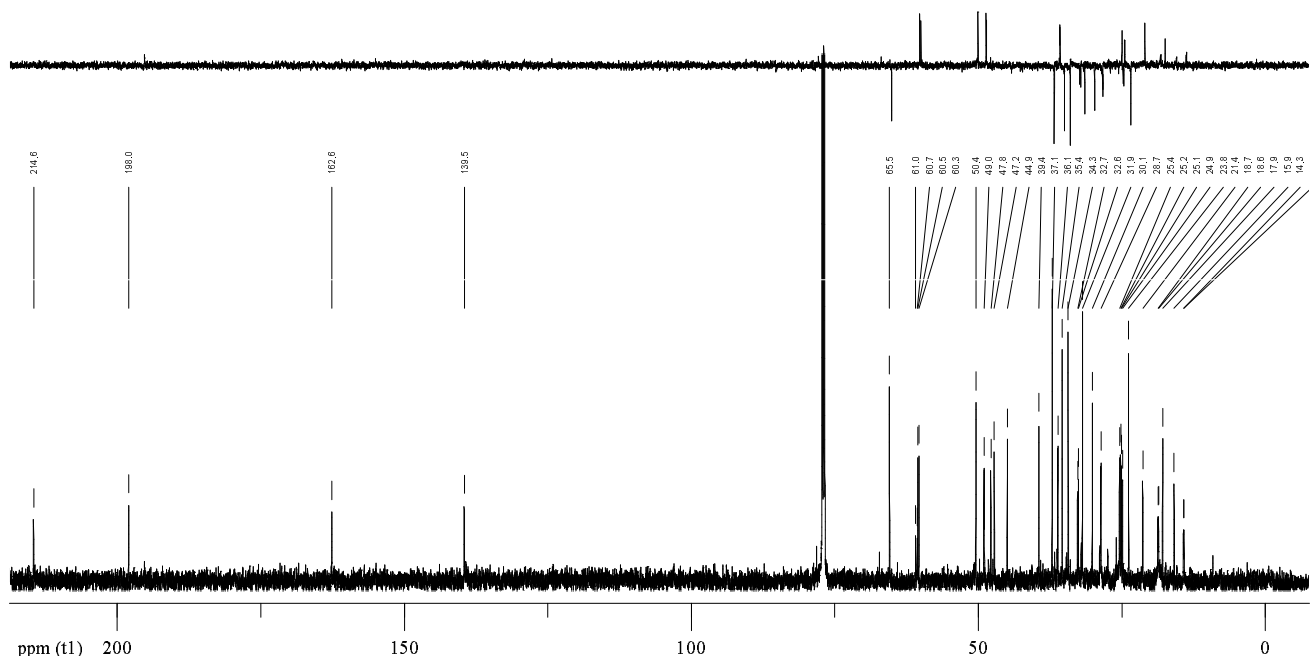

Figure 3-72 ${ }^{13} \mathrm{C}-\mathrm{NMR}$ and DEPT135 Ganoderone $\mathrm{C}$ in $\mathrm{CDCl}_{3}$ (overview)

\subsubsection{2 ${ }^{13} \mathrm{C}-\mathrm{NMR}$ detail}

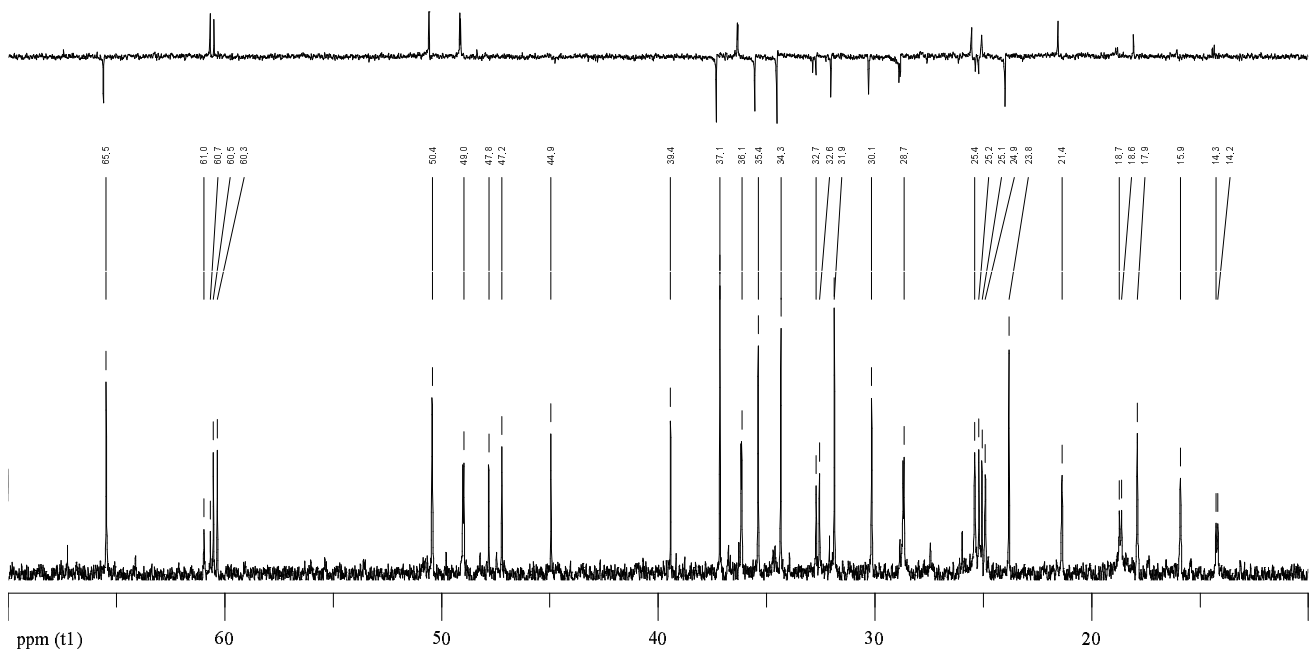

Figure 3-73 ${ }^{13} \mathrm{C}-\mathrm{NMR}$ und DEPT135 Ganoderone $\mathrm{C}$ in $\mathrm{CDCl}_{3}$ (10 to $70 \mathrm{ppm}$ ) 


\subsubsection{HSQC}

\subsubsection{HSQC overview}

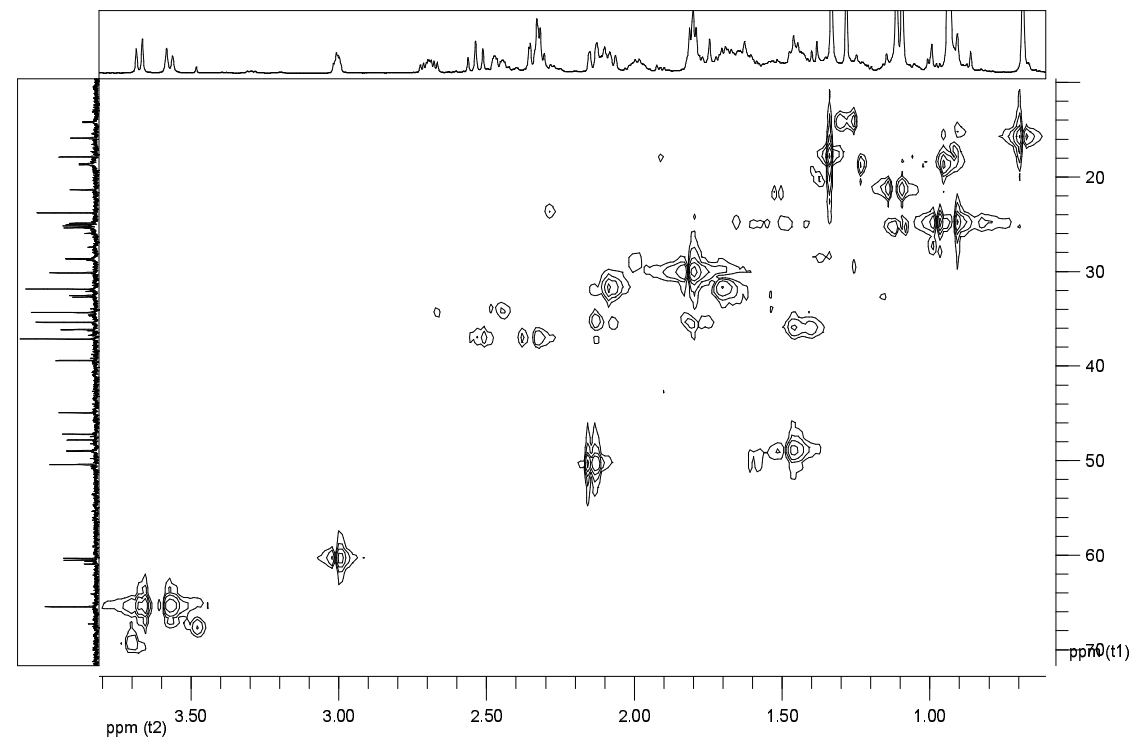

Figure 3-74 HSQC Ganoderone $\mathrm{C}$ in $\mathrm{CDCl}_{3}\left(0.4\right.$ to $5.7\left({ }^{1} \mathrm{H}\right), 9$ to $\left.140 \mathrm{ppm}\left({ }^{13} \mathrm{C}\right)\right)$

\subsubsection{HSQC detail}

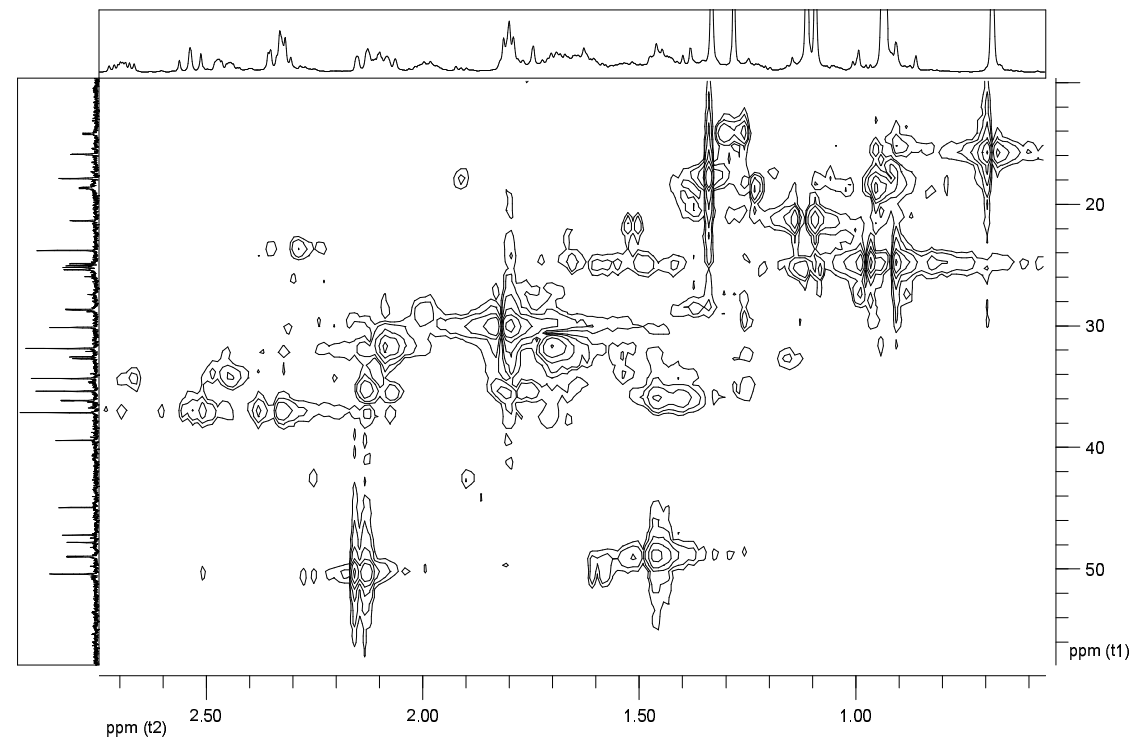

Figure 3-75 HSQC Ganoderone $\mathrm{C}$ in $\mathrm{CDCl}_{3}\left(0.56\right.$ to $2.74\left({ }^{1} \mathrm{H}\right), 9$ to $\left.58 \mathrm{ppm}\left({ }^{13} \mathrm{C}\right)\right)$ 


\subsubsection{HMBC}

\subsubsection{HMBC overview}

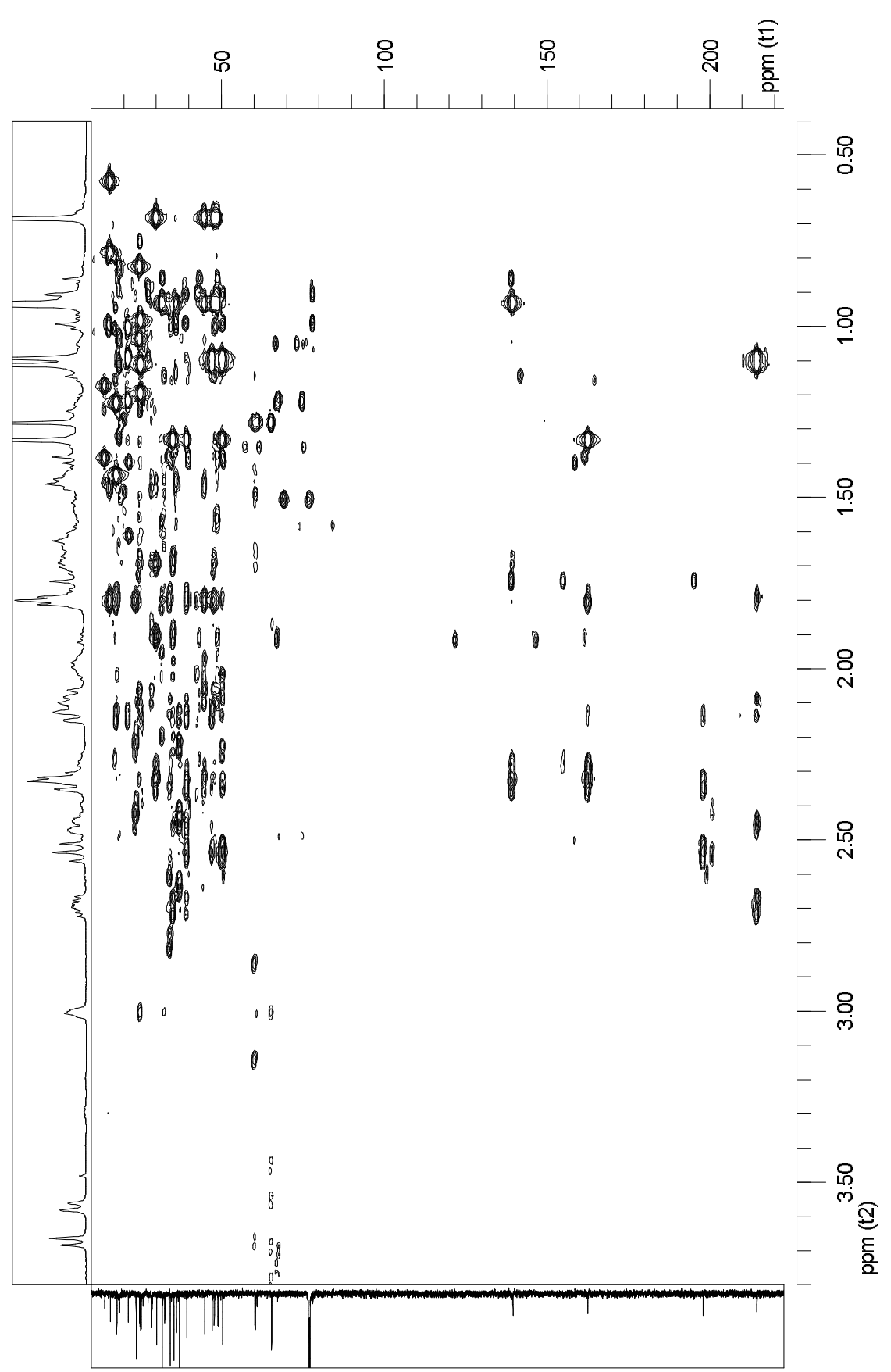

Figure 3-76 HMBC Ganoderone $\mathrm{C}$ in $\mathrm{CDCl}_{3}\left(0.4\right.$ to $3.8\left({ }^{1} \mathrm{H}\right), 10$ to $\left.222 \mathrm{ppm}\left({ }^{13} \mathrm{C}\right)\right)$ 


\subsubsection{HMBC detail}

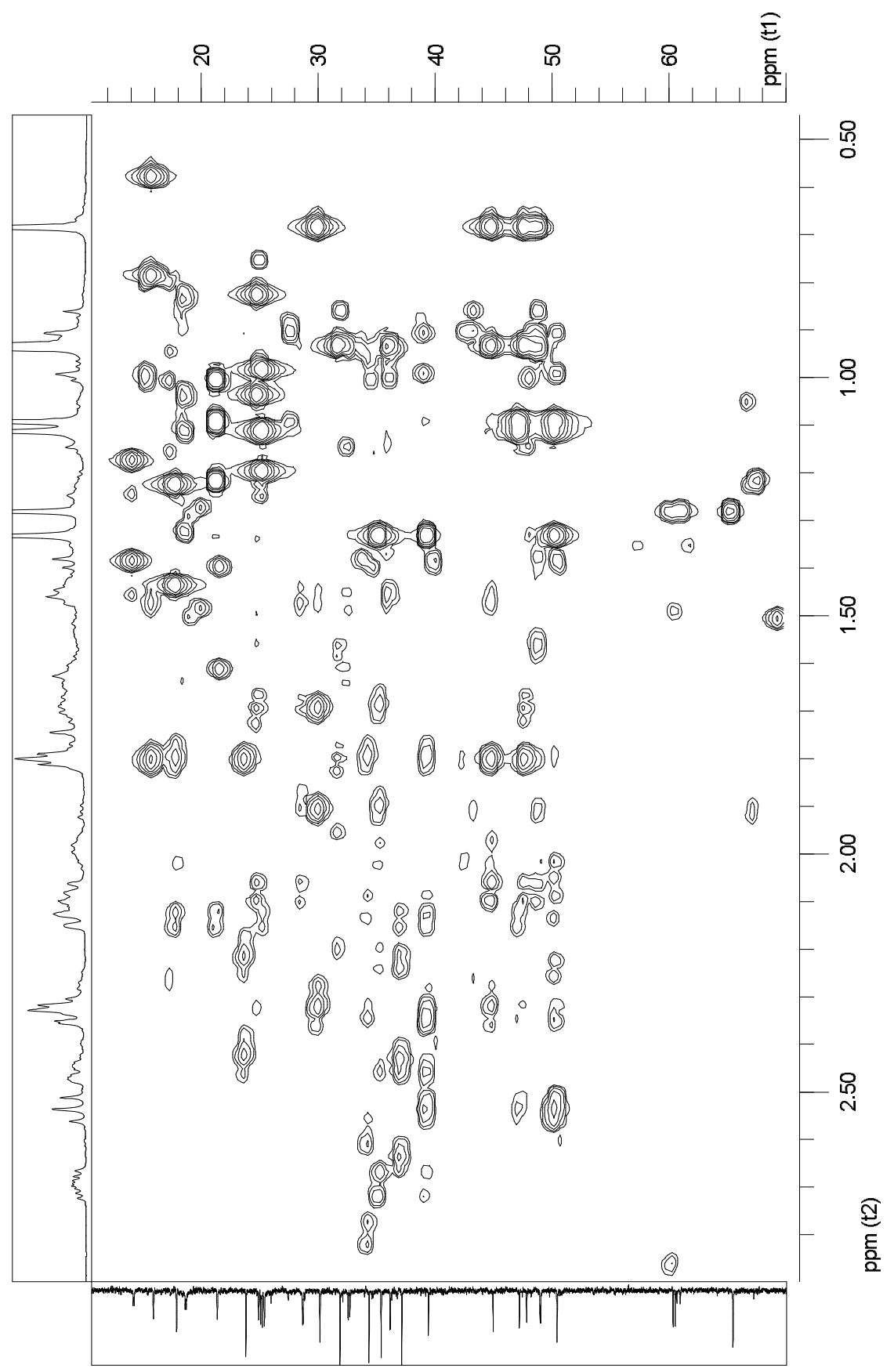

Figure 3-77 HMBC Ganoderone $\mathrm{C}$ in $\mathrm{CDCl}_{3}$ (0.45 to $2.9\left({ }^{1} \mathrm{H}\right), 10$ to $70 \mathrm{ppm}\left({ }^{13} \mathrm{C}\right)$ ) 


\subsubsection{HMBC correlations}

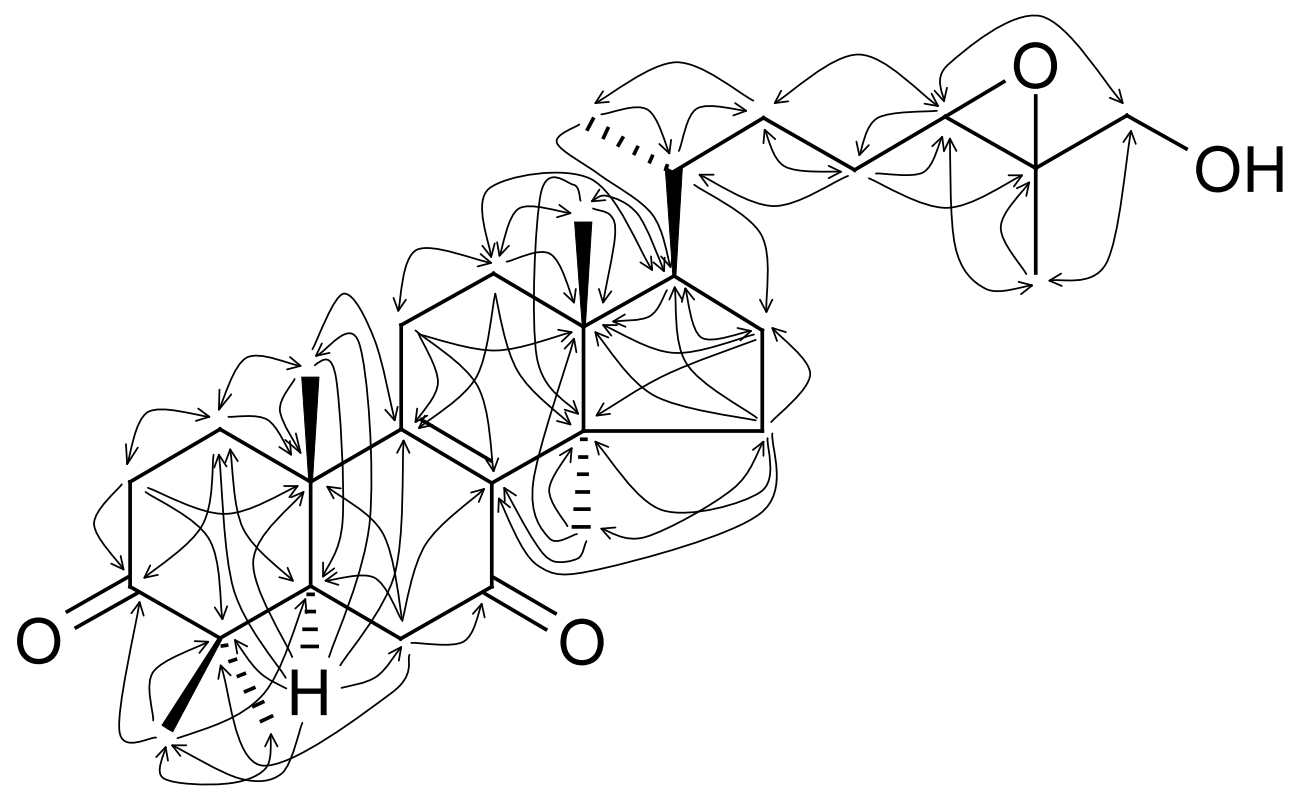

Figure 3-78 HMBC correlations Ganoderone C 


\subsubsection{NOESY}

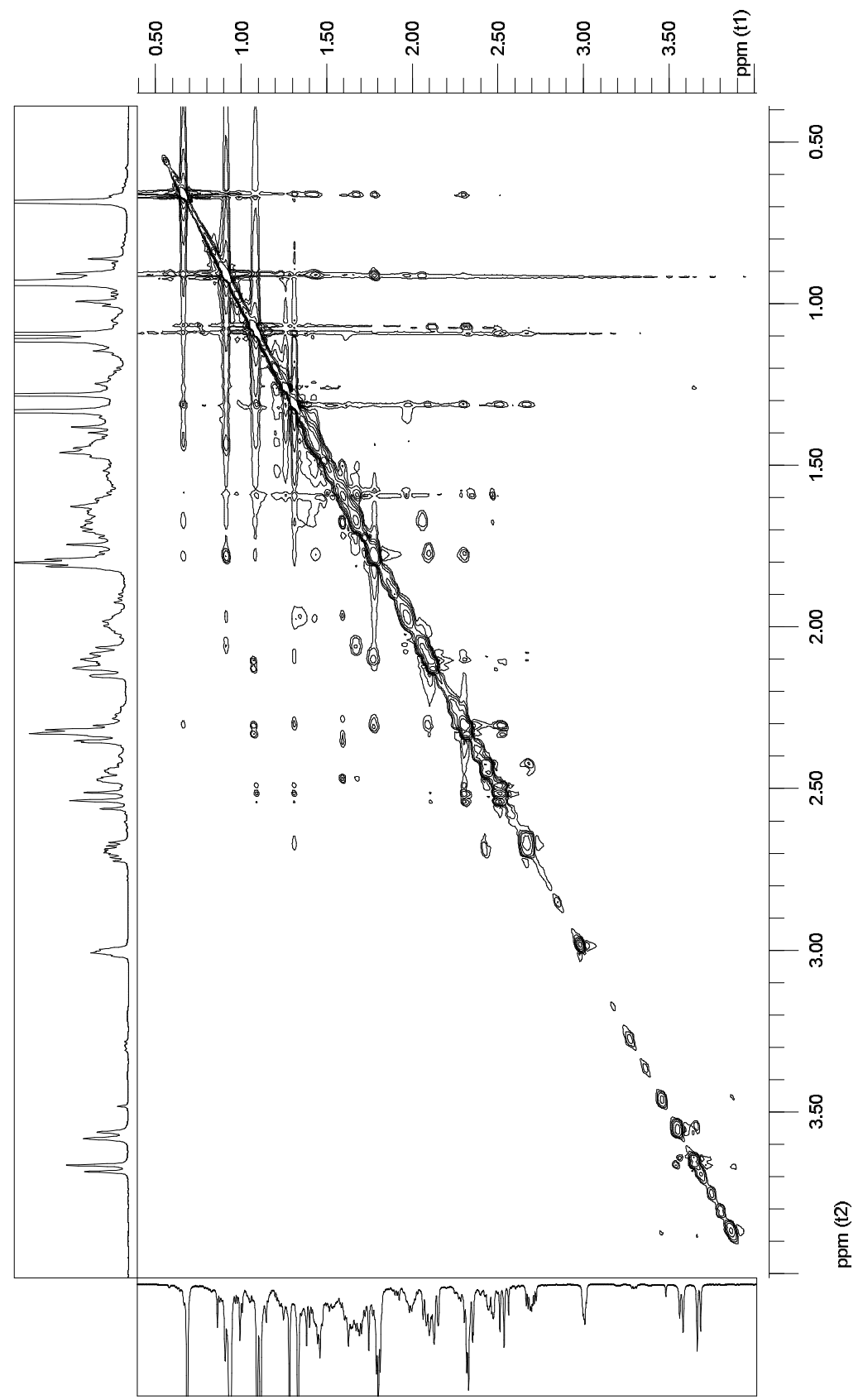

Figure 3-79 NOESY Ganoderone $\mathrm{C}$ in $\mathrm{CDCl}_{3}\left(0.39\right.$ to $\left.4.01\left({ }^{1} \mathrm{H}\right)\right)$ 


\subsection{9 similar natural compounds}

- Epoxyganoderiol $\mathrm{A}^{36}$

- Epoxyganoderiol $\mathrm{B}^{36}$

- Epoxyganoderiol $\mathrm{C}^{36}$

- Ganoderone $\mathrm{A}^{\text {this article }}$

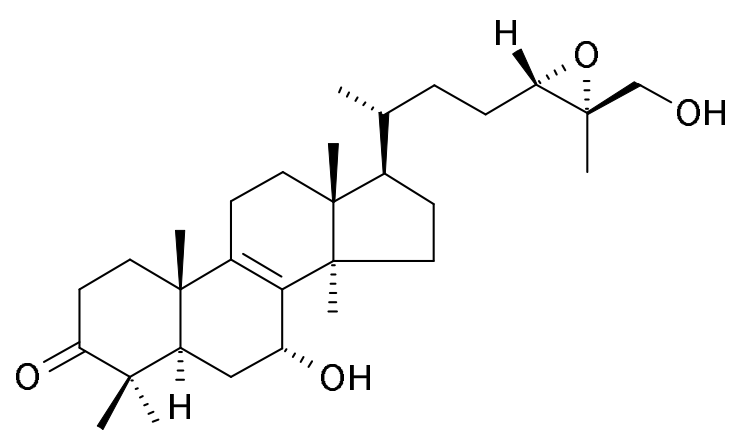

Figure 3-80 Epoxyganoderiol A

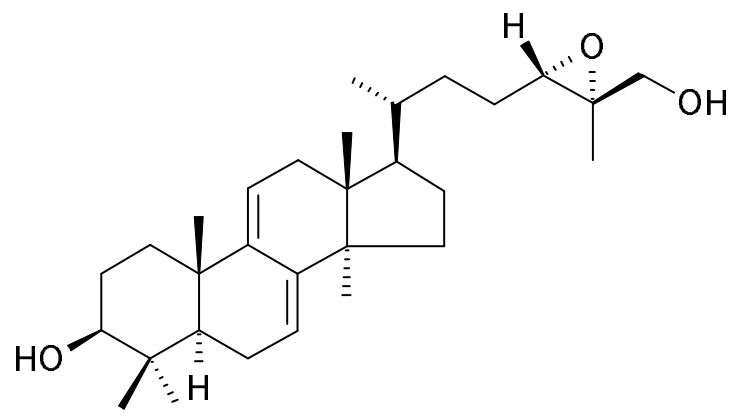

Figure 3-82 Epoxyganoderiol C

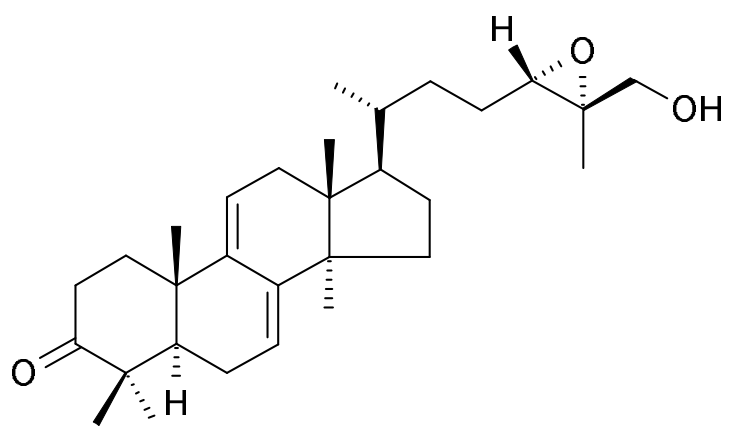

Figure 3-81 Epoxyganoderiol B

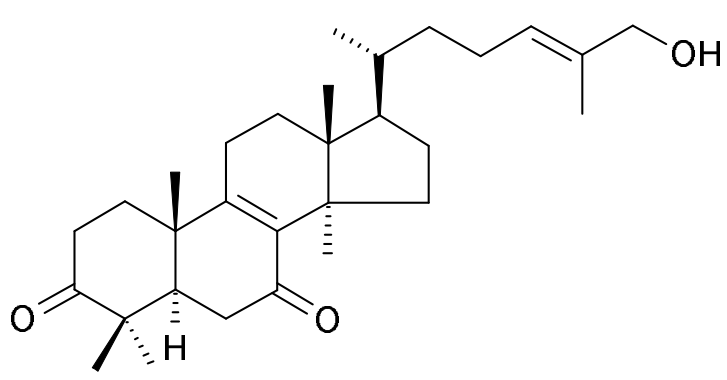

Figure 3-83 Ganoderone A 


\subsection{Applanoxidic acid G}

Isolation see Figure 2-1 on page 2. white amorphous powder. yield $97.5 \mathrm{mg}$ (min. $0.0339 \%$ in dried fruiting bodies of $G$. pfeifferi). TLC: $\mathrm{R}_{\mathrm{f}} 0.21$; fluorescence quenching at $254 \mathrm{~nm}$, weak blue fluorescence at $366 \mathrm{~nm}$; violet with anisaldehyde-sulphuric acid reagent.

HRFTICRMS [M+H] ${ }^{+}$: calcd for $\mathrm{C}_{30} \mathrm{H}_{41} \mathrm{O}_{8}$ : 529.2801; found: 529.2816.

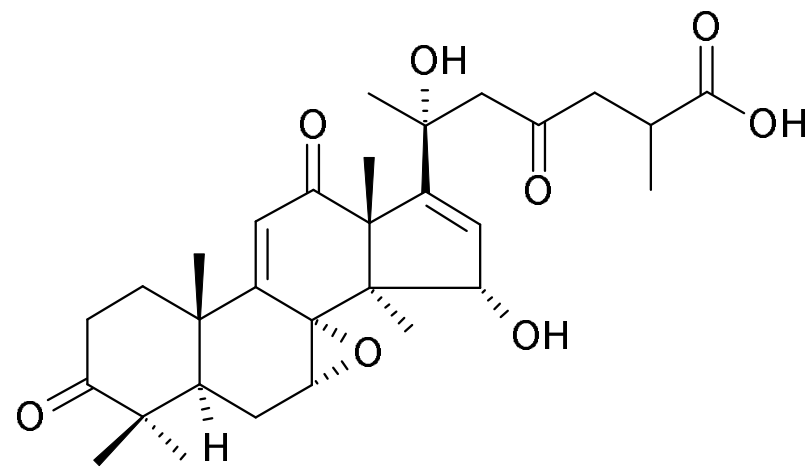

Applanoxidic acid G

\subsubsection{UV-vis}

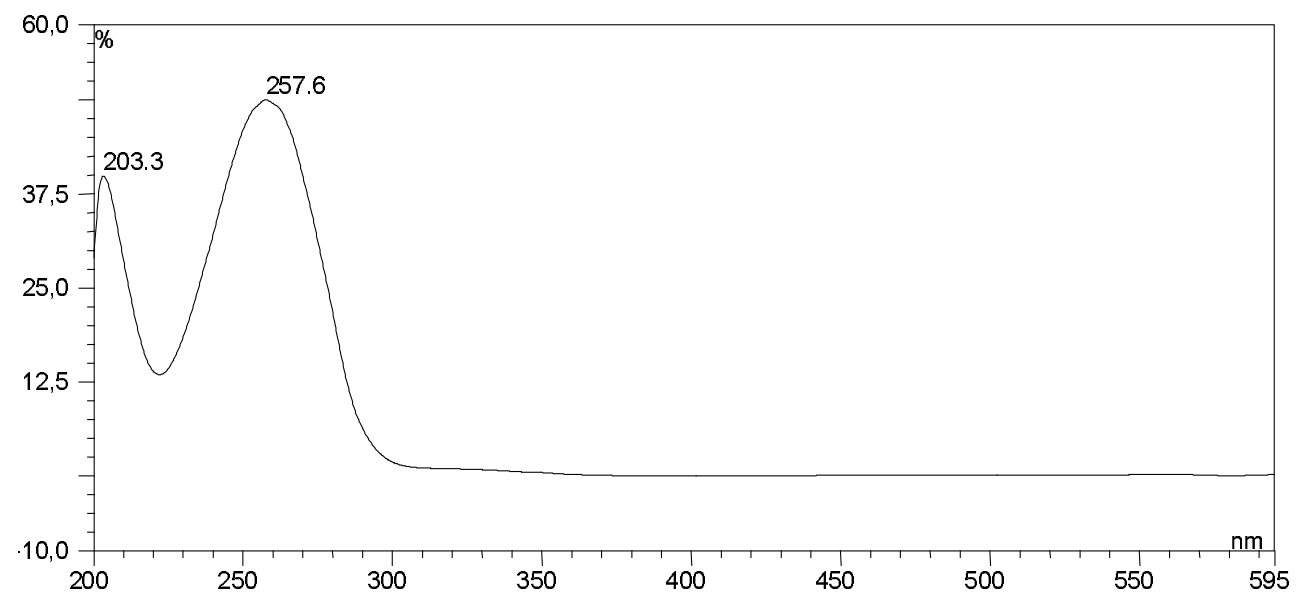

Figure 3-84 UV-vis Applanoxidic acid G 


\subsubsection{EI-MS}

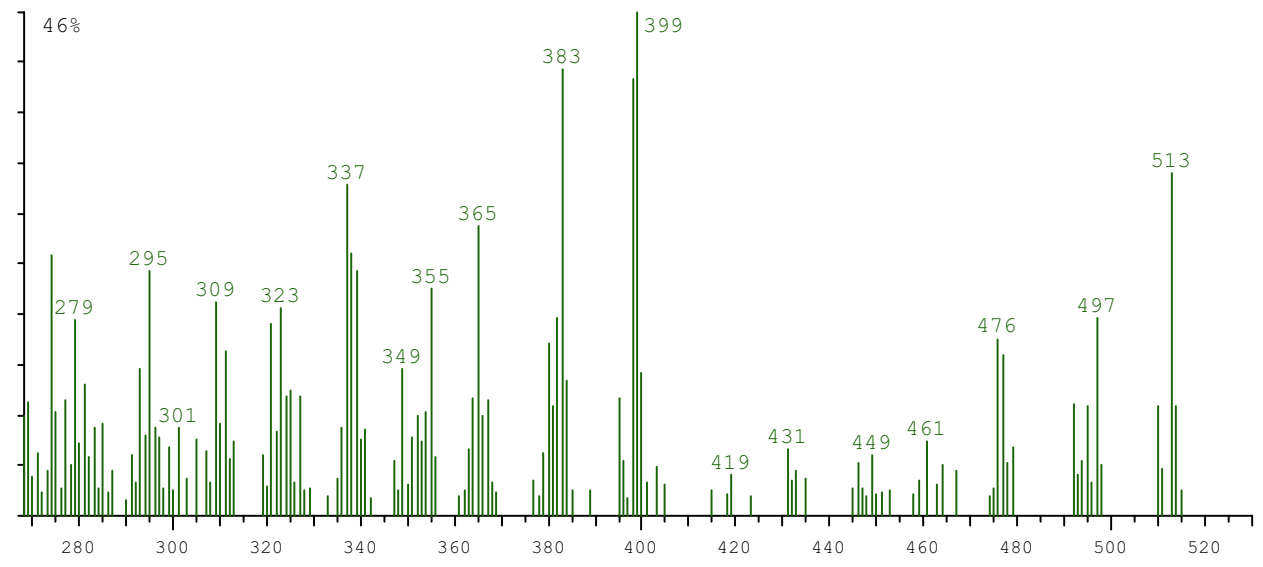

Figure 3-85 EI-MS Applanoxidic acid G

\subsection{3 ${ }^{1} \mathrm{H}-\mathrm{NMR}$}

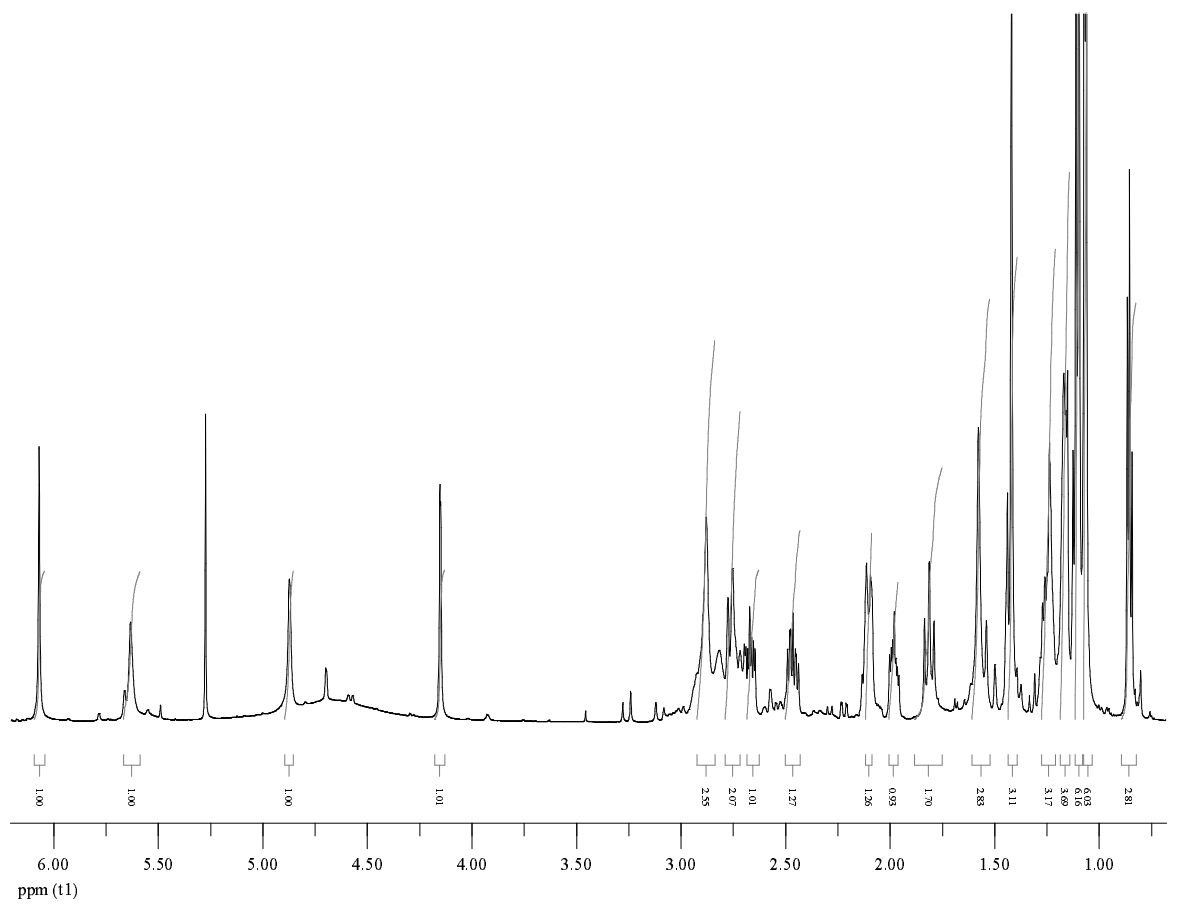

Figure 3-86 ${ }^{1} \mathrm{H}-\mathrm{NMR}$ Applanoxidic acid $\mathrm{G}$ in $\mathrm{CDCl}_{3}$ 


\subsection{4 ${ }^{13} \mathrm{C}-\mathrm{NMR}$}

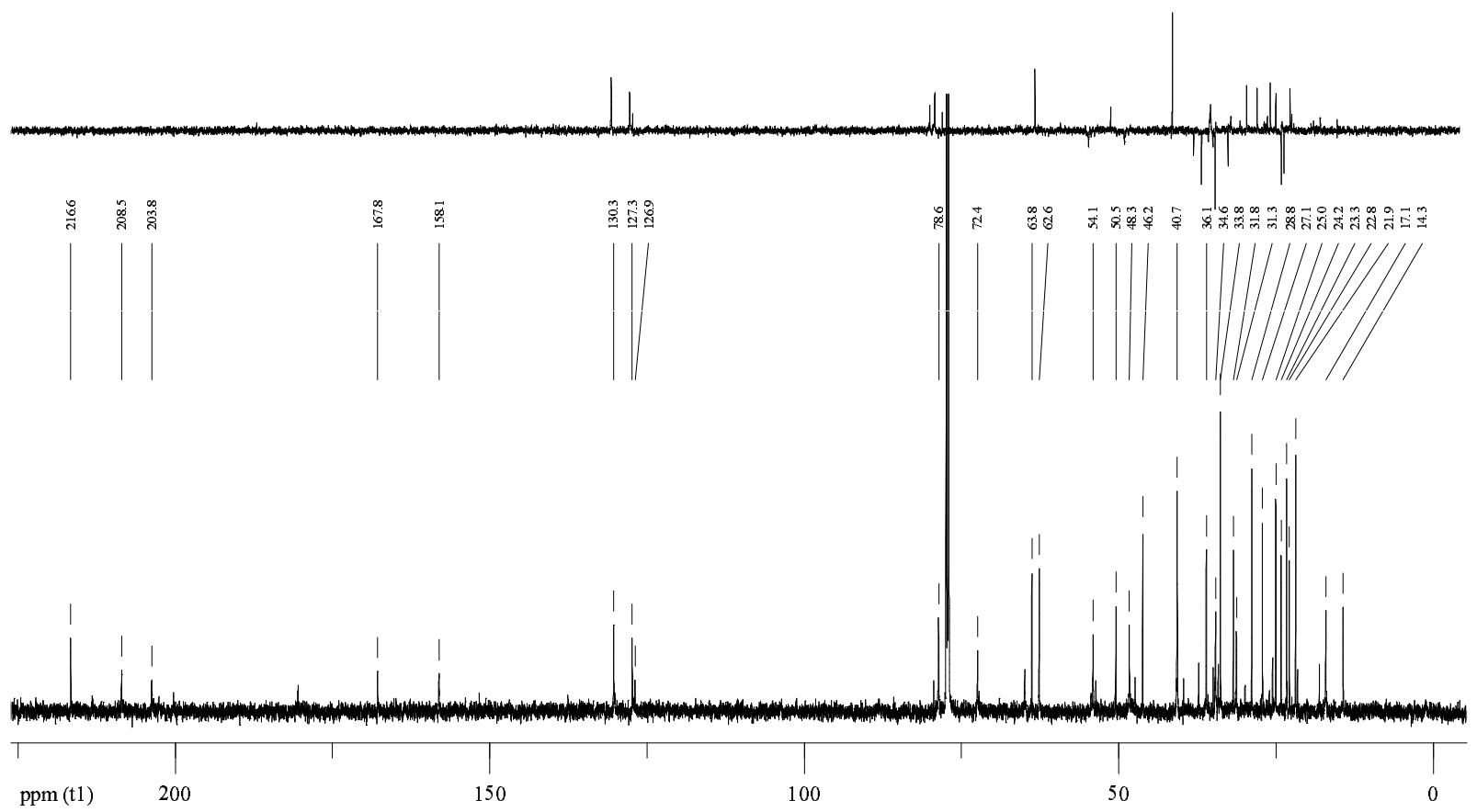

Figure 3-87 ${ }^{13} \mathrm{C}-\mathrm{NMR}$ und DEPT135 Applanoxidic acid $\mathrm{G}$ in $\mathrm{CDCl}_{3}$ 


\subsubsection{Literature}

\begin{tabular}{|c|c|c|c|c|c|}
\hline year & isolated from & yield $(\%)$ & structure analysis & biol. activity & in \\
\hline 1994 & G. applanatum & $\mathrm{n} / \mathrm{a}$ & $\begin{array}{l}\mathrm{mp},[\alpha]_{\mathrm{D}}, \mathrm{UV}-\mathrm{vis}, \mathrm{IR}, \\
\text { EI-MS, }{ }^{1} \mathrm{H}-,{ }^{13} \mathrm{C}-\mathrm{NMR}\end{array}$ & $\begin{array}{l}\text { inhibitory effect on Epstein-Barr } \\
\text { virus-associated early antigen ac- } \\
\text { tivation (mouse skin tumor pro- } \\
\text { moter) }\end{array}$ & 71 \\
\hline 2000 & G. australe & $\mathrm{n} / \mathrm{a}$ & $\mathrm{n} / \mathrm{a}$ & $\begin{array}{l}\text { weak antibacterial activity } \\
\text { against } B . \text { cereus, no activity } \\
\text { against } S . \text { aureus, } E \text {. coli and } P \text {. } \\
\text { aeruginosa }\end{array}$ & 27 \\
\hline 2003 & G. pfeifferi & 0.07 & $\begin{array}{l}\mathrm{mp}, \mathrm{UV}-\mathrm{vis}, \mathrm{IR},{ }^{1} \mathrm{H}- \\
{ }^{13} \mathrm{C}-\mathrm{NMR}, \mathrm{EI}-\mathrm{MS}\end{array}$ & $\begin{array}{l}\text { antiviral activity against in- } \\
\text { fluenza virus type } \mathrm{A}\left(\mathrm{ED}_{50} 0.19\right. \\
\text { mmol/L), no activity against } \\
\text { HSV type } 1\end{array}$ & 69 \\
\hline 2003 & G. annulare & $\mathrm{n} / \mathrm{a}$ & $\mathrm{n} / \mathrm{a}$ & $\begin{array}{l}\text { no antifungal activity against } \\
\text { Microsporum cannis and Tri- } \\
\text { chophyton mentagrophytes }\end{array}$ & 28 \\
\hline 2003 & G. australe & 0.0011 & $\mathrm{n} / \mathrm{a}$ & $\begin{array}{l}\text { cytotoxic for HL-60 cells }\left(\mathrm{IC}_{50}\right. \\
404 \pm 80 \mu \mathrm{M})\end{array}$ & 30 \\
\hline
\end{tabular}

Table 3-10 Literature Applanoxidic acid G 


\subsection{Applanoxidic acid A}

Isolation see Figure 2-1 on page 2. white amorphous powder. yield $47.5 \mathrm{mg}$ (min. $0.0165 \%$ in dried fruiting bodies of G. pfeifferi). TLC: $\mathrm{R}_{\mathrm{f}} 0.10$; fluorescence quenching at $254 \mathrm{~nm}$, no fluorescence at $366 \mathrm{~nm}$; yellow with anisaldehyde-sulphuric acid reagent.

HRFTICRMS [M+H] ${ }^{+}$: calcd for $\mathrm{C}_{30} \mathrm{H}_{41} \mathrm{O}_{7}$ : 513.2853; found: 513.2863 .

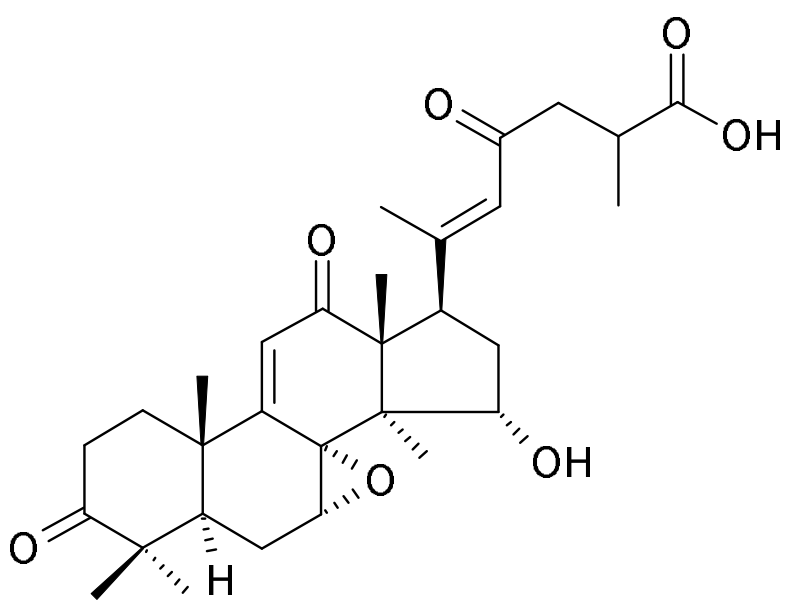

Applanoxidic acid A

\subsubsection{UV-vis}

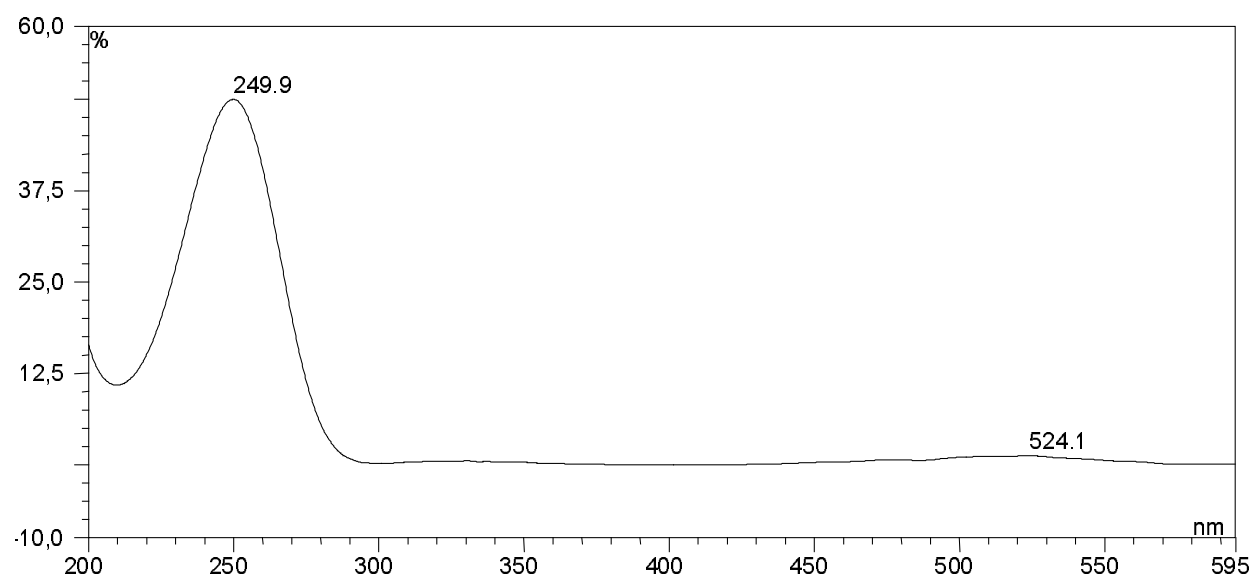

Figure 3-88 UV-vis Applanoxidic acid A 


\subsubsection{EI-MS}

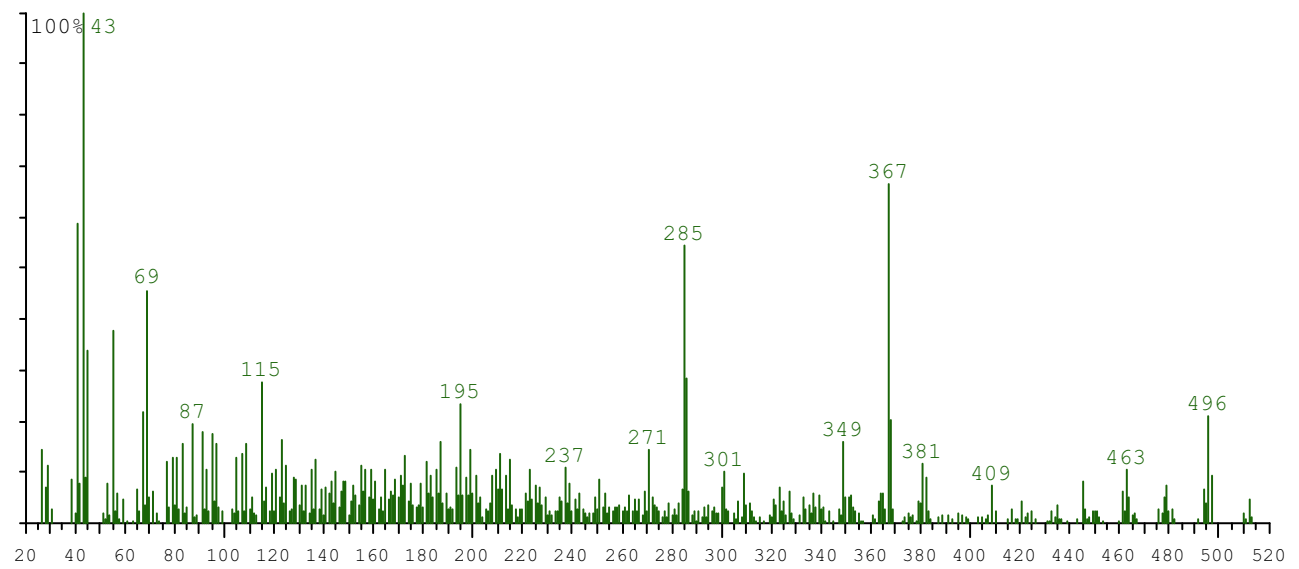

Figure 3-89 EI-MS Applanoxidic acid A

\subsection{3 ${ }^{1} \mathrm{H}-\mathrm{NMR}$}

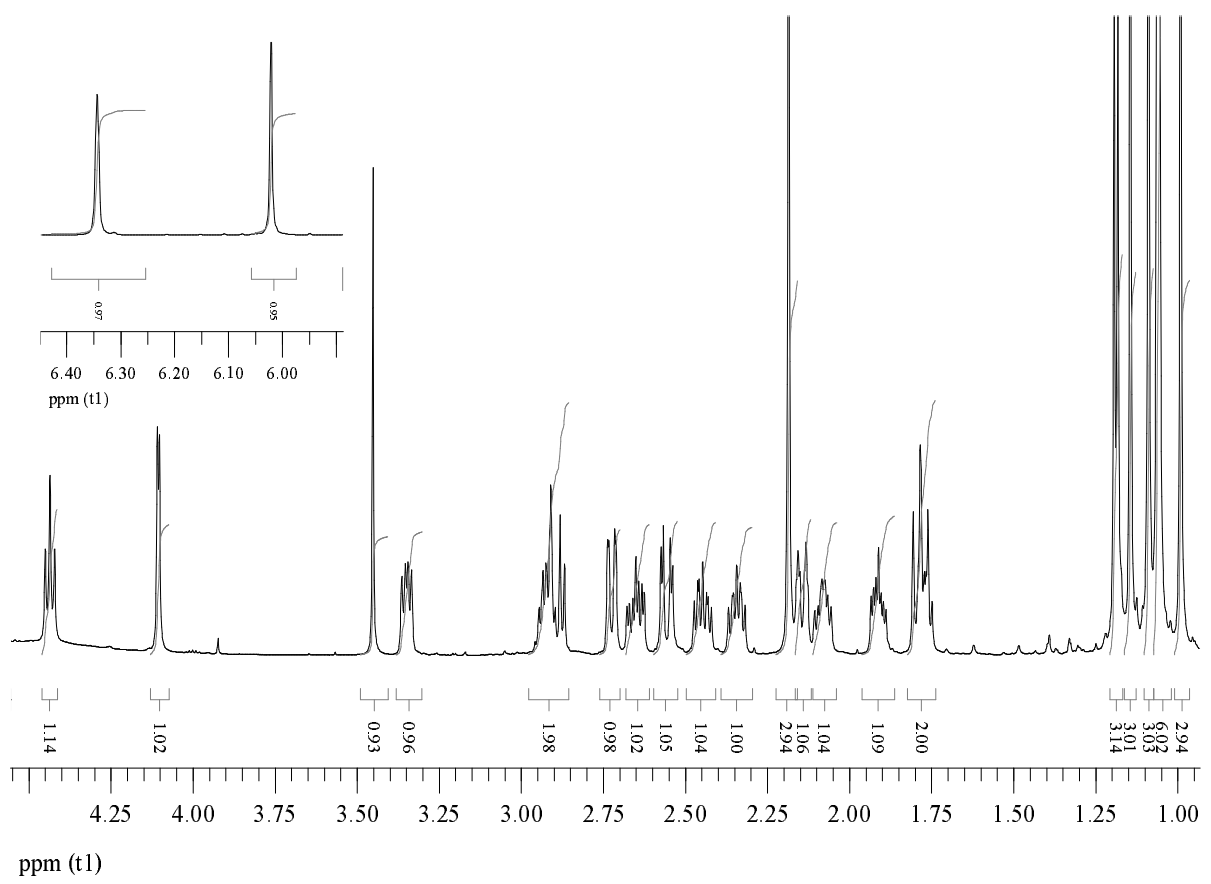

Figure 3-90 $\quad{ }^{1} \mathrm{H}-\mathrm{NMR}$ Applanoxidic acid $\mathrm{A}$ in $\mathrm{CDCl}_{3}$ 


\subsection{4 ${ }^{13} \mathrm{C}-\mathrm{NMR}$}

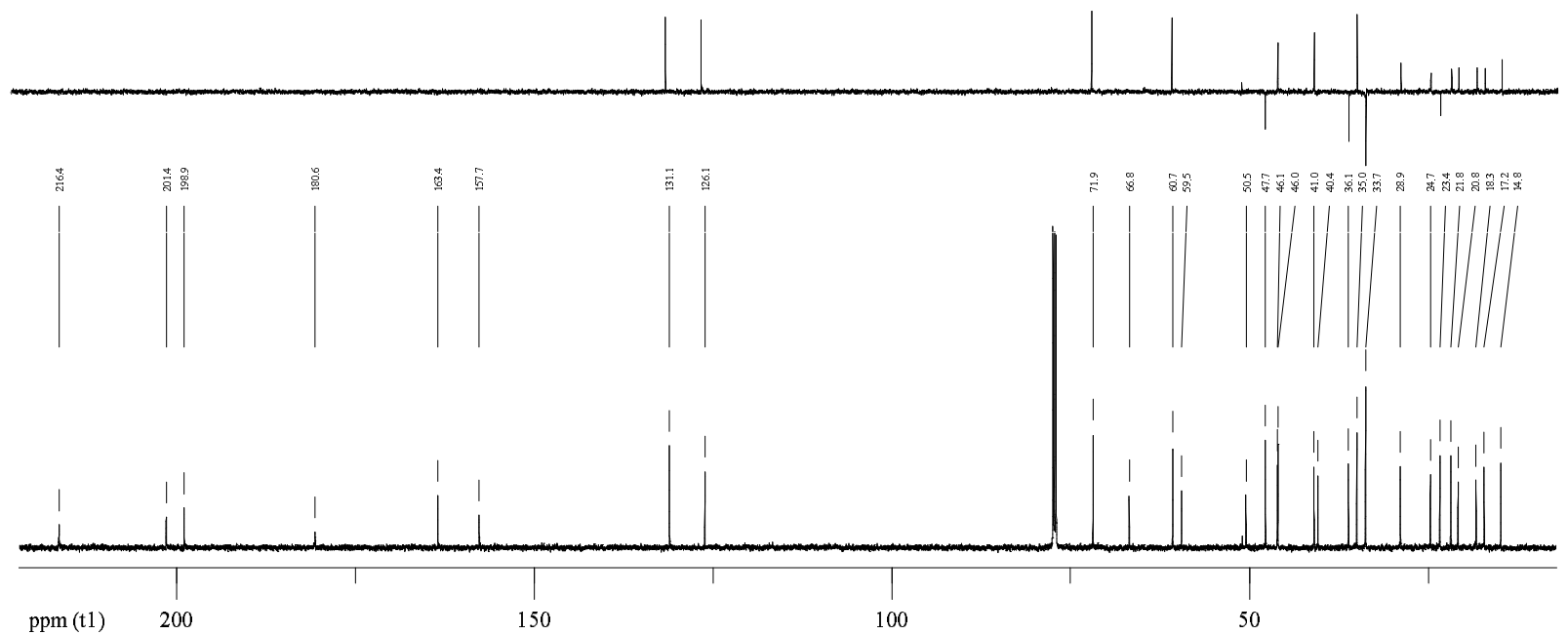

Figure 3-91 ${ }^{13} \mathrm{C}-\mathrm{NMR}$ und DEPT135 Applanoxidic acid $\mathrm{A}$ in $\mathrm{CDCl}_{3}$

\subsubsection{Literature}

\begin{tabular}{|c|c|c|c|c|c|}
\hline year & isolated from & yield $(\%)$ & structure analysis & biol. activity & in \\
\hline 1991 & G. applanatum & 0.009 & $\begin{array}{l}\mathrm{mp},[\alpha]_{\mathrm{D}}, \mathrm{UV}, \mathrm{IR}, \mathrm{EI}- \\
\mathrm{MS},{ }^{1} \mathrm{H}-,{ }^{13} \mathrm{C}-\mathrm{NMR}\end{array}$ & $\begin{array}{l}\text { inhibitory effect on Epstein-Barr } \\
\text { virus-associated early antigen ac- } \\
\text { tivation (mouse skin tumor pro- } \\
\text { moter) }\end{array}$ & 70 \\
\hline 2000 & G. australe & $\mathrm{n} / \mathrm{a}$ & $\mathrm{n} / \mathrm{a}$ & $\begin{array}{l}\text { weak antibacterial activity } \\
\text { against } B . \text { cereus, no activity } \\
\text { against } S . \text { aureus, E. coli and } P \text {. } \\
\text { aeruginosa }\end{array}$ & 27 \\
\hline 2003 & G. annulare & $\mathrm{n} / \mathrm{a}$ & $\mathrm{n} / \mathrm{a}$ & $\begin{array}{l}\text { no antifungal activity against } \\
\text { Microsporum cannis and Tri- } \\
\text { chophyton mentagrophytes }\end{array}$ & 28 \\
\hline 2003 & G. australe & 0.0017 & $\mathrm{n} / \mathrm{a}$ & $\begin{array}{l}\text { cytotoxic for HL-60 cells }\left(\mathrm{IC}_{50}\right. \\
132 \pm 22 \mu \mathrm{M})\end{array}$ & 30 \\
\hline
\end{tabular}

Table 3-11 Literature Applanoxidic acid A 


\section{References}

1. Ripperger, H.; Budzikiewicz, H. Phytochemistry 1975, 14, 2297-2298.

2. Jain, A. C.; Gupta, S. K. Phytochemistry 1984, 23, 686-687.

3. Protiva, J.; Skorkovská, H.; Urban, J.; Vystrcil, A. Collect. Czech. Chem. Commun. 1980, 45, 2710-2713.

4. Lu, W.; Adachi, I.; Kano, K.; Yasuta, A.; Toriizuka, K.; Ueno, M.; Horikoshi, I. Chem. Pharm. Bull. 1985, 33, 5083-5087.

5. Lin, C. N.; Tome, W. P.; Won, S. J. Phytochemistry 1990, 29, 673-675.

6. Gan, K. H.; Kuo, S. H.; Lin, C. N. J. Nat. Prod. 1998, 61, 1421-1422.

7. Gonzalez, A. G.; Leon, F.; Rivera, A.; Padron, J. I.; Gonzalez-Plata, J.; Zuluaga, J. C.; Quintana, J.; Estevez, F.; Bermejo, J. J. Nat. Prod. 2002, 65, 417-421.

8. Morigiwa, A.; Kitabatake, K.; Fujimoto, Y.; Ikekawa, N. Chem. Pharm. Bull. 1986, 34, 3025-3028.

9. Miyahara, R.; Yoshimoto, T.; Asawa, K. Mokuzai Gakkaishi 1987, 33, 416-422.

10. Morimoto, H.; Imada, I.; Murata, T.; Matsumoto, N. Liebigs Ann. Chem. 1967, 708, $230-240$.

11. Schulte, K. E.; Ruecker, G.; Fachmann, H. Tetrahedron Lett. 1968, 46, 4763-4764.

12. Yuan, D.; Mori, J.; Komatsu, K.; Makino, T.; Kano, Y. Biol. Pharm. Bull. 2004, 27, $867-870$. 
13. Kwon, H. C.; Zee, S. D.; Cho, S. Y.; Choi, S. U.; Lee, K. R. Arch. Pharmacal Res. 2002, $25,851-855$.

14. Zhang, Y.; Mills, G.; Nair, M. G. J. Agric. Food Chem. 2002, 20, 7581-7585.

15. White, J. D.; Taylor, S. I. J. Am. Chem. Soc. 1970, 92, 5811-5813.

16. Yuan, D.; Yamamoto, K.; Bi, K. S.; Zhang, P.; Liu, F.; Kano, Y. J. Pharm. Soc. Jap. 2003, 123, 53-62.

17. Daftary, R. D.; Pomeranz, Y.; Cooks, R. G.; Wolfe, N. L. Experientia 1970, 26, 1056-1057.

18. Bindler, G. N.; Piade, J. J.; Schulthess, D. Beiträge zur Tabakforschung International 1988, $14,127-134$.

19. Tanaka, N.; Hosoi, K.; Tanaka, D.; Takahashi, M. Chem. Pharm. Bull. 1996, 44, 843-846.

20. Kobayashi, M.; Krishna, M. M.; Ishida, K.; Anjaneyulu, V. Chem. Pharm. Bull. 1992, 40, $72-74$.

21. Chen, R.; Wang, Y.; Yu, D. Zhiwu Xuebao 1991, 33, 65-68.

22. Gonzalez, A. G.; Leon, F.; Rivera, A.; Munoz, C. M.; Bermejo, J. J. Nat. Prod. 1999, 62, 1700-1701.

23. Kahlos, K.; Kangas, L.; Hiltunen, R. Acta Pharmaceutica Fennica 1987, 96, 33-40.

24. Kahlos, K.; Hiltunen, R.; Kangas, L. Fitoterapia 1989, 60, 166-168.

25. Keller, A. C.; Maillard, M. P.; Hostettmann, K. Phytochemistry 1996, 41, 1041-1046.

26. Barrero, A. F.; Oltra, J. E.; Poyatos, J. A.; Jimenez, D.; Oliver, E. J. Nat. Prod. 1998, 61, 1491-1496.

27. Smania, A., J.; Delle Monache, F.; Smania, E. F. A.; Cuneo, R. S. Int. J. Med. Mushrooms 1999, 1, 325-330.

28. Smania, E. F. A.; Delle Monache, F.; Smania, A.; Yunes, R. A.; Cuneo, R. S. Fitoterapia 2003, 74, 375-377.

29. Kac, D.; Barbieri, G.; Falco, M. R.; Seldes, A. M.; Gros, E. G. Phytochemistry 1984, 23, 2686-2687. 
30. Leon, F.; Valencia, M.; Augusto, R.; Nieto, I.; Quintana, J.; Estevez, F.; Bermejo, J. Helv. Chim. Acta 2003, 86, 3088-3095.

31. Lehmkuhl-Gerber, A.; Smania Junior, A.; Delle Monache, F.; Biacchi Junior, N.; Smania, E. F. A. Int. J. Med. Mushrooms 2000, 2, 303-311.

32. Gonzalez, A. G.; Bermejo, J.; Toledo, M. F. J.; Mediavilla, P. M. J.; Beltran, T. E. Anales de Quimica, Serie C: Quimica Organica y Bioquimica 1986, 82, 149-151.

33. Keller, A. C.; Keller, J.; Maillard, M. P.; Hostettmann, K. Phytochemistry 1997, 46, 963-965.

34. Ma, J. Y.; Ye, Q.; Hua, Y. J.; Zhang, D. C.; Cooper, R.; Chang, M. N.; Chang, J. Y.; Sun, H. H. J. Nat. Prod. 2002, 65, 72-75.

35. Lin, C. N.; Fann, Y. F.; Chung, M. I. Phytochemistry 1997, 46, 1143-1146.

36. Nishitoba, T.; Sato, H.; Oda, K.; Sakamura, S. Agric. Biol. Chem. 1988, 52, 211-216.

37. Gao, J. J.; Min, B. S.; Ahn, E. M.; Nakamura, N.; Lee, H. K.; Hattori, M. Chem. Pharm. Bull. 2002, 50, 837-840.

38. Komoda, Y.; Nakamura, H.; Ishihara, S.; Uchida, M.; Kohda, H.; Yamasaki, K. Chem. Pharm. Bull. 1985, 33, 4829-4835.

39. Arisawa, M.; Fujita, A.; Saga, M.; Fukumura, H.; Hayashi, T.; Shimizu, M.; Morita, N. J. Nat. Prod. 1986, 49, 621-625.

40. Arisawa, M.; Fujita, A.; Hayashi, T.; Shimizu, M.; Morita, N.; Kikuchi, T.; Kadota, S.; Tezuka, Y. J. Nat. Prod. 1988, 51, 54-59.

41. Della Greca, M.; Mangoni, L.; Molinaro, A.; Monaco, P.; Previtera, L. Gazz. Chim. Ital. 1990, 120, 391-392.

42. Della Greca, M.; Ferrara, M.; Fiorentino, A.; Monaco, P.; Previtera, L. Phytochemistry 1998, 49, 1299-1304.

43. Anis, I.; Ahmed, S.; Malik, A.; Yasin, A.; Choudary, M. I. Chem. Pharm. Bull. 2002, 50, 515-518. 
44. Kuria, K. A. M.; Chepkwony, H.; Govaerts, C.; Roets, E.; Busson, R.; De Witte, P.; Zupko, I.; Hoornaert, G.; Quirynen, L.; Maes, L.; Janssens, L.; Hoogmartens, J.; Laekeman, G. J. Nat. Prod. 2002, 65, 789-793.

45. Macias, F. A.; Simonet, A. M.; Galindo, J. C. G. J. Chem. Ecol. 1997, 23, 1781-1803.

46. Cantrell, C. L.; Rajab, M. S.; Franzblau, S. G.; Fronczek, F. R.; Fischer, N. H. Planta Med. 1999, 65, 732-734.

47. Sheikh, Y. M.; Djerassi, C. Tetrahedron 1974, 30, 4095-4103.

48. Gunatilaka, A. A. L.; Gopichand, Y.; Schmitz, F. J.; Djerassi, C. J. Org. Chem. 1981, 46, 3860-3866.

49. Windaus, A.; Brunken, J. Liebigs Ann. Chem. 1928, 460, 225-235.

50. Wieland, P.; Prelog, V. Helv. Chim. Acta 1947, 30, 1028-1030.

51. Lindequist, U.; Lesnau, A.; Teuscher, E.; Pilgrim, H. Pharmazie 1989, 44, 579-580.

52. Narbe, G.; Lindequist, U.; Teuscher, E.; Franke, P.; Vainiotalo, P.; Basner, R. Pharmazie 1991, 46, 738-740.

53. Patocka, J. Acta Med. 1999, 42, 123-125.

54. Fujimoto, H.; Nakayama, M.; Nakayama, Y.; Yamazaki, M. Chem. Pharm. Bull. 1994, 42, 694-697.

55. El-Mekkawy, S.; Meselhy, M. R.; Nakamura, N.; Tezuka, Y.; Hattori, M.; Kakiuchi, N.; Shimotohno, K.; Kawahata, T.; Otake, T. Phytochemistry 1998, 49, 1651-1657.

56. Mizushina, Y.; Watanabe, I.; Togashi, H.; Hanashima, L.; Takemura, M.; Ohta, K.; Sugawara, F.; Koshino, H.; Esumi, Y.; Uzawa, J.; Matsukage, A.; Yoshida, S.; Sakaguchi, K. Biol. Pharm. Bull. 1998, 21, 444-448.

57. Kim, S. W.; Park, S. S.; Min, T. J.; Yu, K. H. Bull. Korean Chem. Soc. 1999, 20, 819-823.

58. Bok, J. W.; Lermer, L.; Chilton, J.; Klingeman, H. G.; Towers, G. H. N. Phytochemistry 1999, 51, 891-898. 
59. Kim, D. S.; Jeong, H. J.; Bhat, K. P. L.; Park, S. Y.; Kang, S. H.; Yoo, E. H.; Lee, M.; Lee, H. W.; Krueger, R. J.; Kim, D. S. H. L. Planta Med. 2000, 66, 78-79.

60. Nam, K. S.; Jo, Y. S.; Kim, Y. H.; Hyun, J. W.; Kim, H. W. Life Sci. 2001, 69, 229-237.

61. Kuo, Y. C.; Weng, S. C.; Chou, C. J.; Chang, T. T.; Tsai, W. J. Br. J. Pharmacol. 2003, 140, 895-906.

62. Li, G.; Xu, M. L.; Lee, C. S.; Woo, M. H.; Chang, H. W.; Son, J. K. Arch. Pharmacal Res. 2004, 27, 829-833.

63. Lin, C. N.; Kuo, S. H.; Won, S. J. Phytochemistry 1993, 32, 1549-1551.

64. Chiang, H. C.; Ho, C. C. Huaxue 1990, 48, 253-258.

65. Sviridonov, V. N.; Strigina, L. I. Khim. Prir. Soedin. 1976, 669.

66. Hata, K.; Hori, K.; Miura, N.; Sakamoto, K.; Takahashi, S. Natural Medicines 2001, 55, 304-307.

67. Chiang, H. C.; Chu, S. C. J. Chin. Chem. Soc. (Taipei, Taiwan) 1991, 38, 71-76.

68. Yeh, S. F.; Lee, K. C.; Shiao, M. S. Proceedings of the National Science Council, Republic of China, Part A: Physical Science and Engineering 1987, 11, 129-134.

69. Mothana, R. A. A.; Ali, N. A. A.; Jansen, R.; Wegner, U.; Mentel, R.; Lindequist, U. Fitoterapia 2003, 74, 177-180.

70. Chairul,; Tokuyama, T.; Hayashi, Y.; Nishizawa, M.; Tokuda, H.; Chairul, S. M.; Hayashi, Y. Phytochemistry 1991, 30, 4105-4109.

71. Chairul,; Chairul, S. M.; Hayashi, Y. Phytochemistry 1994, 35, 1305-1308. 